\title{
11
}

\section{Ceramic assemblages from excavations on Viti Levu, Beqa-Ugaga and Mago Island}

\author{
Geoffrey Clark \\ Department of Archaeology and Natural History, The Australian National University
}

\section{Introduction}

This chapter describes the ceramic collections from Navatu 17A, Karobo, Votua (1996), Natunuku, Malaqereqere, Volivoli II, Volivoli III, Ugaga Island and Kulu Bay. The EPF ceramics from the nine sites consisted of 54,522 sherds, weighing $295.8 \mathrm{~kg}$. The ceramics were analysed at different intensities, with Navatu, Karobo and Ugaga reported in a PhD thesis (Clark 2000), and those from Votua, Natunuku and Qaranioso II published, although not always in detail, in several papers (Anderson and Clark 1999; Anderson et al. 2000; Clark and Anderson 2001; Clark et al. 2001). Details of the ceramics from the nine sites are discussed further below, with assemblage descriptions divided in two.

First, each assemblage is described in terms of sherd type (number of plain body, decorated body, rims, necks) and weight by excavation level, along with the occurrence and distribution of decoration and surface modification. Where assemblages could be identified by stratigraphy/age, the ceramic assemblages are considered separately, as at Navatu 17A, where three assemblages were defined. At sites such as Kulu Bay, Ugaga Island and Natunuku, where ceramics were mixed and contained older and more recent pottery, the entire ceramic assemblage is described. When the size of rim fragments allows, an attempt is made to identify sherds to a particular vessel form, with a description of reconstructed vessels from Navatu, Ugaga, Votua and Karobo in Clark (2000) and Clark et al. (2001). Selected sherds are figured in line drawings and photographs, but the large size of the collection precludes full depiction of all diagnostic sherds in the EPF collections. 


\section{Vessel forms}

Rim sherds were classed into one of 10 generalised vessel forms (Figure 116, see Clark 2000:61-64).

Jars - Form 1

1A. Everted-direct rim - indirect body contour

1B. Abruptly thickened then thinning rim - indirect body contour

Jars were the main vessel form identified in the EPF collection's assemblages. They are characterised by a small to significant degree of rim eversion $\left(0-85^{\circ}\right)$ and have a restricted orifice (indirect rim-body contour). Form 1B jars are distinguished by abruptly thickened rims, and include the important collar-rim vessels associated with Lapita assemblages.

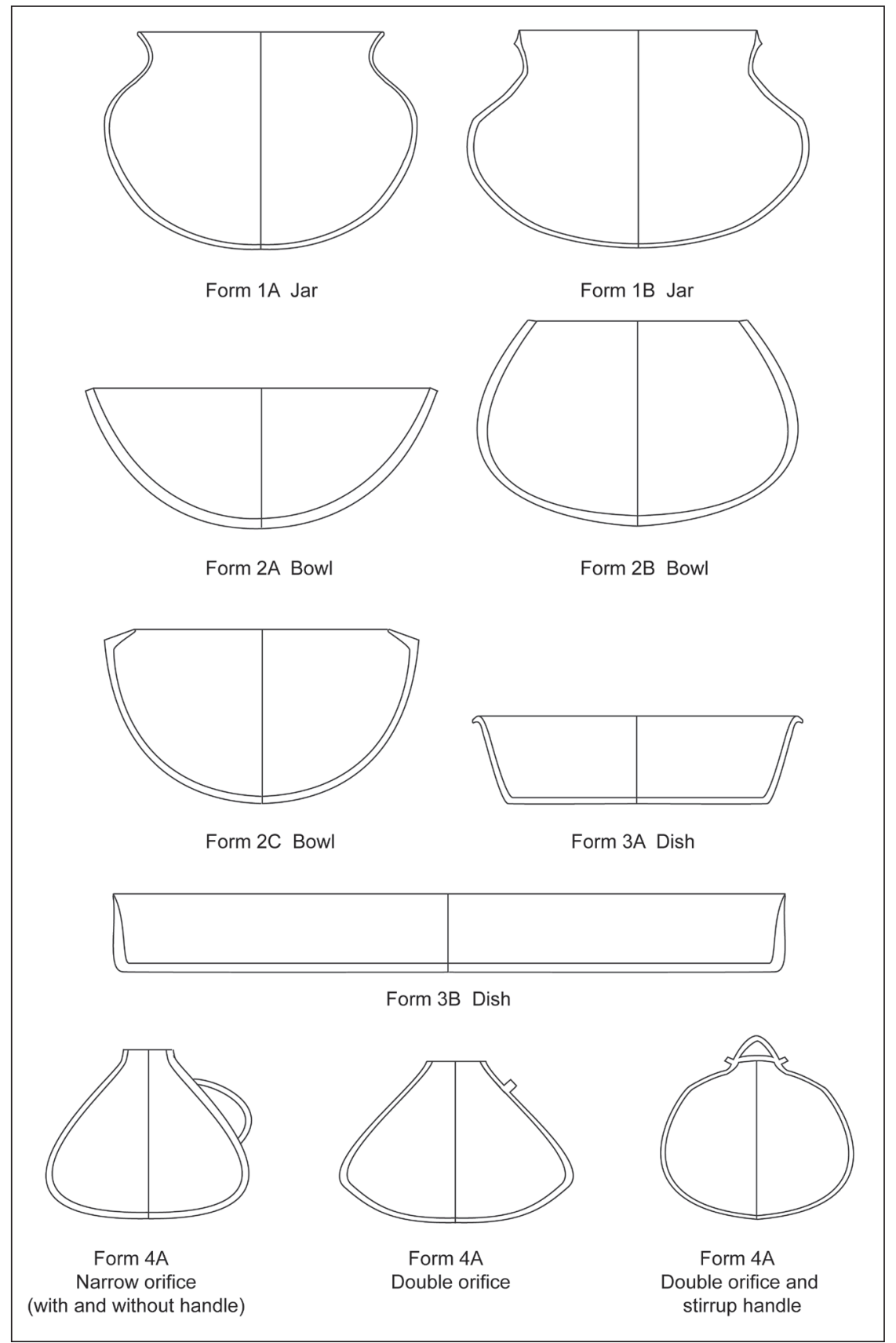

Figure 116. Generalised vessel forms. Form 1 Jars, Form 2 bowls, Form 3 dishes and platters, Form 4 narrow orifice. 
Bowls - Form 2

2A. Everted rim - direct rim-body contour

2B. Inverted rim - direct rim-body contour

2C. Inverted rim - indirect rim-body contour

These bowls have a direct or indirect rim-body contour, but do not have necks (Rice 1987:216). Three vessel sub-forms varying in rim orientation and rim contour were identified. Bowls were the second most common vessel form, although they were substantially fewer in number than Form 1 vessels.

Flat-based vessels (dishes) - Form 3

3A. Flat-based - small diameter $(<30 \mathrm{~cm})$

3B. Flat-based - large diameter $(>45 \mathrm{~cm})$

Vessels with flat bases were divided into two sub-forms to distinguish between temporally and morphologically distinct forms. Few flat-based vessels were present in any assemblage.

Narrow orifice (10 cm diameter or less) - Form 4

4A. Single orifice and indirect rim-body contour (with and without a handle)

4B. Double orifice

4C. Double orifice and stirrup handle

The fourth vessel form includes vessels with narrow apertures/orifices. Three sub-forms were established to encompass the range of vessels present. Vessels with double apertures emerging from a stirrup handle dominate this group.

\section{Navatu ceramics}

\section{Navatu Layer 1 ceramics (Table 43)}

Of the 5070 sherds recovered from Layer 1, 4.2\% are decorated. Carved paddle-impressed sherds (cross-hatch, parallel rib, curvilinear) occurred throughout Layer 1 in low frequencies $(\mathrm{n}=39)$. These sherds almost certainly derived from Layers 2 or 4 , as they are small and eroded in comparison to other Layer 1 sherds, and surface modification of this kind was otherwise restricted to Layer 4. Other decorated sherds likely to be intrusive include an applied fillet with end-tool impressing, and a body sherd with two rows of diagonal fingernail impressions; both sherds from basal Layer 1 contexts. The most frequent kind of vessel decoration consists of shell impressions $(2.2 \%)$. The impressions appear to have been made with marine-shell valves, but other tools could produce similar results. Four kinds of shell impression are included in the 'shell-impressed' category. These are wavy comb, linear comb, vertical comb and angled comb (see Figure 117).

Wavy comb refers to the curvilinear pattern imparted from a toothed comb, possibly with the valve edge from a marine bivalve such as Anadara or Gafrarium. The sinusoidal patterns have a short amplitude of $1 \mathrm{~cm}$ or less and were placed on the interior/exterior rim and body of Form $1 \mathrm{~A}$ jars. On the interior of the rim, wavy comb is bordered by horizontal bands of linear incising. On the exterior vessel, surface wavy comb occurs on the shoulder, where it was bordered by horizontal bands of linear-comb or angled-comb incising. The most frequent type of linear comb are bands of incised lines. The incising consists of three to seven lines that circle the neck, shoulder or inner rim. Linear-comb incising was also used to border a geometric area that was left undecorated or was filled with vertical or angled comb. Vertical comb was applied in two ways. In the first, the toothed shell edge penetrated the clay, leaving a set of small and irregular wedge-shaped impressions (Gifford 1951:Plate 22m). The impressions were stacked in linear 
Table 43. Navatu, Trench B, Layer 1 ceramics: Sherd type and decoration by depth.

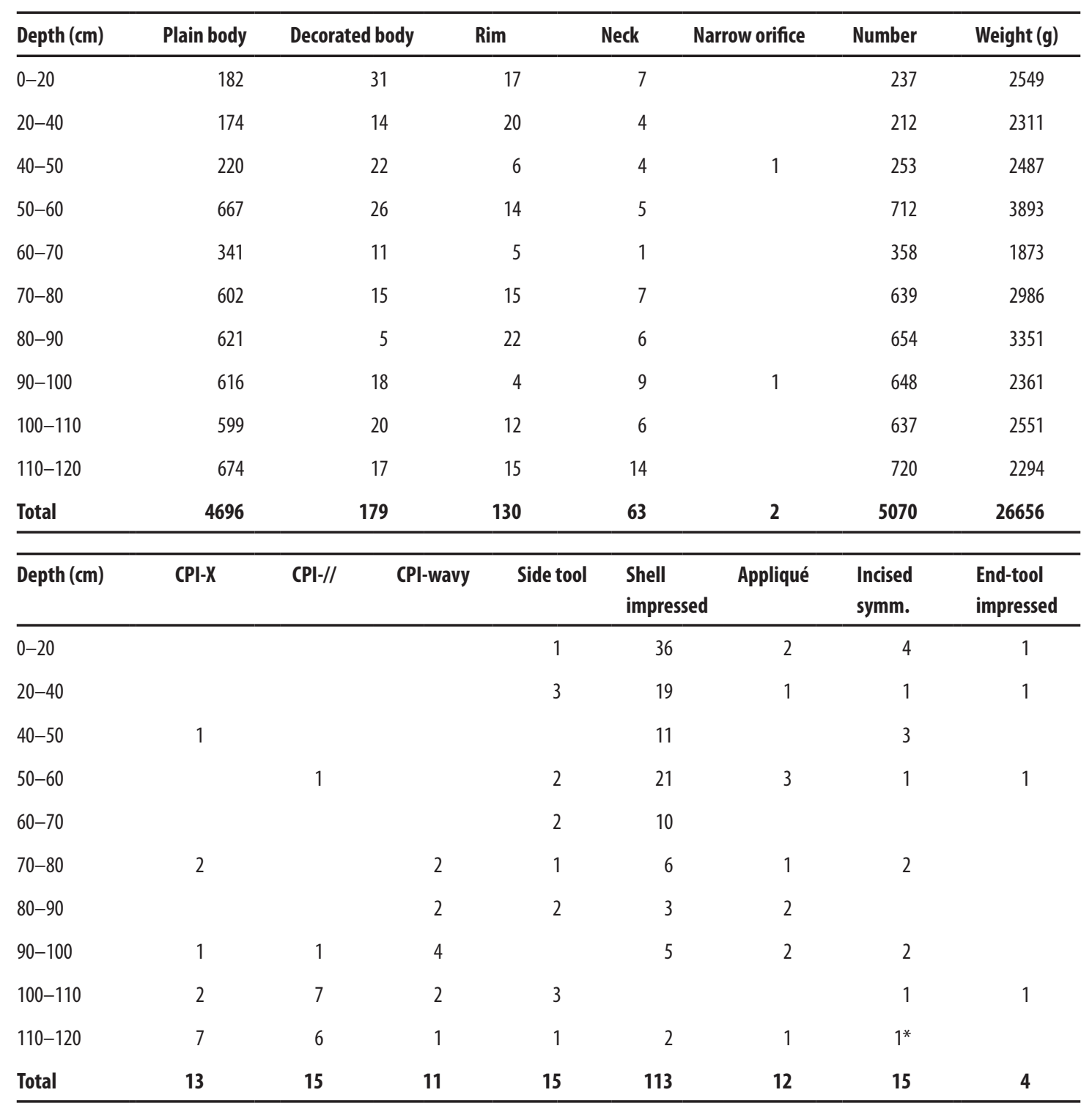

$\mathrm{CPI}=$ carved paddle impressed, $/ /=$ parallel-rib, $\mathrm{X}=$ cross-hatch, wavy=curvilinear. * =sherd with asymmetric incision.

columns and bordered by linear-comb incision on the shoulder and neck. By pushing the shell deeper into the vessel surface, a short undulating line was formed and these impressions were applied in columns and diagonal rows (Figure 117). This was a common method for decorating the lip of Form 1A vessels. Angled comb was similar to vertical comb, except the valve edge was pressed into the clay at an angle, rather than vertically. This created a strongly textured surface, which was applied in block form as columns, rows and other geometric shapes.

Only 12 cases $(0.2 \%)$ of appliqué were found in Layer 1 . These consist of fillets, buttons, buttresses, small ovals and clay crescents $(1.2 \mathrm{~cm}$ long). Buttresses consist of an applied clay band around the neck that were finger gouged or modelled. Buttons and fillets were placed on neck and shoulder regions, but the designs cannot be determined. Of the 15 cases of sidetool notching, three are likely to derive from Layer 4, as indicated by their distinctive oval form. The remaining side-tool notching is on rims from medium-to-large Form 1A vessels. The notching consists of deep serrations on outer and inner-lip circumferences. Outer-edge serrations are usually deeper and wider spaced $(0.3-0.5 \mathrm{~cm})$ than those on the inner lip. The 


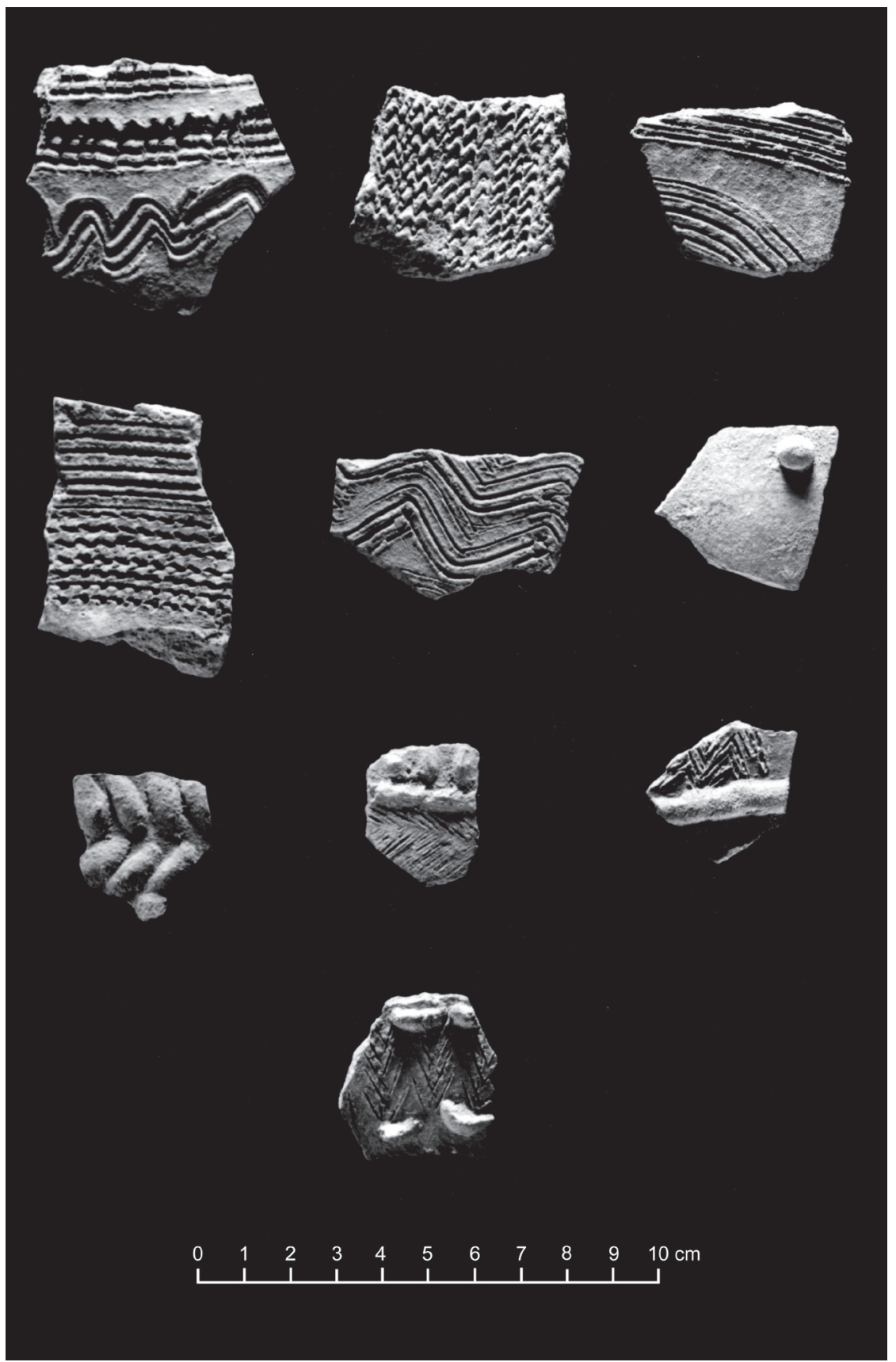

Figure 117. Navatu 17 , Layer 1 sherds decorated with shell impression, appliqué and incision.

serrations were made with the toothed edge of a shell valve. There are 14 sherds $(0.3 \%)$ with symmetric incision. Thin ' $\mathrm{V}$ ' shaped incisions were found on the lip of a Form $1 \mathrm{~A}$ jar and Form $4 \mathrm{~A}$ vessel. Cross-hatch incisions occur on a Form $1 \mathrm{~A}$ vessel and on the rim of an unknown vessel form with a thick rim and round lip. Incised hatching in the form of large $(2 \mathrm{~cm})$ and small $(0.4 \mathrm{~cm})$ diamond patterns was found on two body sherds. The remaining sherds have incised chevrons or partial chevrons. These were placed on the inner-rim surface of Form $1 \mathrm{~A}$ vessels and on an unknown vessel form with a thin wall, where the incisions were bordered by horizontal fillets and oval buttons.

Form 1A jars: Almost 93\% of rims identifiable to a vessel form are assessed as coming from Form 1A vessels. Rim courses are concave (70\%) or straight, and the rim profile is parallel $(37 \%)$ or gradually thinning (44\%). Remaining rims thicken towards the lip $(n=9)$. Lip shape is limited, with most having flat/flat-rounded form (89\%) and the balance 
round/pointed. Decoration was identified on 26 rims. Shell impressing, either across the lip surface or vertically against the lip edge, is the most common decoration $(\mathrm{n}=19)$. Incision (chevron and hatching) and appliqué make up the remainder. Mean rim eversion is $30^{\circ}$ (range $11-72^{\circ}$ ) and average rim course thickness is $7.3 \mathrm{~mm}$. Rim height varies from $16 \mathrm{~mm}$ to $85 \mathrm{~mm}$, with a mean of $37 \mathrm{~mm}$. The neck inclination from 17 sherds has a mean of $103^{\circ}$. The average diameter of 44 rims is $24 \mathrm{~cm}$ (range 12-36 cm). There are two forms of Form $1 \mathrm{~A}$ jar. The first has a rim profile that is parallel or thinned/thickened gradually. Two of the rims belong to large vessels and have interior and exterior rim decoration. The other rim form is a thickened and inwardly bevelled lip, marked by crescent notching or incising.

Form 2A bowls: Two Form 2A vessels were identified. The rims have parallel profiles and flat/flat-rounded lips, with rim eversion angles of $31^{\circ}$ and $42^{\circ}$. Lip decoration consists of a raised modelled ridge and a faint central groove. Estimated bowl diameters are $24 \mathrm{~cm}$ and $30 \mathrm{~cm}$, and it is likely that these vessels represent the ethnographically known dari used in the kava (yaqona) ceremony. Both vessel sherds were in the $0-20 \mathrm{~cm}$ spit.

Form 4 narrow-orifice vessels: Sherds from two narrow-orifice vessels were found in Layer 1 at depths of $40-50 \mathrm{~cm}$ and $80-90 \mathrm{~cm}$. These vessels have short everted rims, with external lip diameters of ca. $10 \mathrm{~cm}$. Lips are flat-rounded and $1 \mathrm{~cm}$ wide. The vessels are decorated with incised chevrons on the lips and fingernail impressions on the buttressed necks. The body shapes were difficult to determine due to the small neck-body portion, and could be ovoid or globular.

\section{Navatu Layer 2 ceramics (Table 44)}

Only 281 sherds were found in Layer 2 and most of these (90\%) are undecorated body sherds. Relief decoration identical to that found in Layer 4 was found on five relatively small and eroded sherds. Side-tool notching on two rims from Form 1A vessels and on a carinated sherd is also similar to decoration found in Layer 4 (see below). Buttressing below the neck of a Form 1A vessel and hatched incising on a rim have parallels with decorated sherds found in Layer 1.

Table 44. Navatu, Trench B, Layer 2 ceramics: Sherd type and decoration by depth.

\begin{tabular}{llllllll}
\hline Depth $(\mathbf{c m})$ & Plain body & Decorated body & Rim & Neck & Carinated & Number & Weight $(\mathbf{g})$ \\
\hline $120-140 \sim$ & 254 & 13 & 9 & 4 & 1 & 281 & 1363 \\
\hline Depth $(\mathrm{cm})$ & CPI-X & CPI-// & CPI- wavy & Side tool & Appliqué & Incised symm. & End-tool impressed \\
\hline $120-140 \sim$ & 2 & 2 & 1 & 1 & 1 & 1 & 1 \\
\hline
\end{tabular}

\section{Navatu Layer 4 ceramics (Table 45)}

The highest frequency of sherds with surface modification (24\%) was found in the Layer 4 assemblage. Paddle relief of various kinds (cross-hatch, parallel rib and curvilinear) is dominant, and smaller numbers of sherds are decorated with other techniques (symmetric incising, end tool and side tool). The cross-hatch group includes relief of diamond, square and rectangular shapes. The combined grouping is necessary, as many Navatu sherds have overlapping or faint impressions that obscure the original form. Sherds with cross-hatch relief make up $7.2 \%$ of the Layer 4 assemblage. The width and height dimensions of the cross-hatch patterns are 3-11.4 $\mathrm{mm}$, with most impressions $7-8 \mathrm{~mm}$ in size (Figure 118). The depth of the pattern varies from faint surface markings to raised ridges 3-4 $\mathrm{mm}$ high. Vessel forms marked with cross-hatch relief include Form 1A and Form 2B vessels (Gifford 1951:Plate 22x). Sherds with parallel relief total 
$5.0 \%$ of the Layer 4 assemblage. Like other kinds of relief applied with a carved paddle, parallel ribs varied in size, depth and location on a vessel. The spacing of parallel ridges ranges from 0.3 $\mathrm{cm}$ to $1.5 \mathrm{~cm}$ and ridge width from $1 \mathrm{~mm}$ to $4 \mathrm{~mm}$ (Figure 118).

Carinated sherds are decorated above or below the carination line at an angle of 40-65 to the vertical (Figure 119). Linear relief was applied in horizontal and diagonal lines to Form $2 \mathrm{~A}$ and $2 \mathrm{~B}$ vessels. The body of Form $1 \mathrm{~A}$ vessels might have been marked by parallel ribs but, except for the presence of thick body sherds with parallel relief, there is little evidence, either in the 1996 assemblage or in Gifford's excavation report, to support this. Of 1852 sherds found in Layer 4, 5.3\% are marked with a raised sinusoidal or curvilinear pattern, a pattern referred to as wavy or zig-zag by previous researchers (Gifford 1951; Shaw 1967; Frost 1970). The wavy impressions are most regular when applied to the shoulder of Form 1A jars (Gifford 1951:Plate 26) and Form 2 bowls. These display shallow to deep impressions $1 \mathrm{~mm}$ to $4 \mathrm{~mm}$ in height, with spacing between ridges of $0.8 \mathrm{~cm}$ to $1.5 \mathrm{~cm}$ (Figure 118). Reverse impressions from shoulder sherds indicate that flat-rounded grooves about $5.2 \mathrm{~mm}$ to $7.5 \mathrm{~mm}$ in width were carved into the paddle in a smooth curvilinear pattern.

Shaw (1967) suggested that the curvilinear impressions could have been made by pressing the subdermal plates from a large turtle into the vessel. This appears unlikely, as the depressions on subdermal bones are much smaller (see Harrisson 1965) than most wavy designs and have

Table 45. Navatu, Trench B, Layer 4 ceramics: Sherd type and decoration by depth.

\begin{tabular}{|c|c|c|c|c|c|c|c|c|c|c|c|c|c|}
\hline \multirow{2}{*}{$\frac{\text { Depth (cm) }}{180-190}$} & \multirow{2}{*}{$\begin{array}{c}\text { Plain body } \\
63\end{array}$} & \multicolumn{2}{|c|}{ Decorated body } & \multirow{2}{*}{\multicolumn{2}{|c|}{$\frac{\operatorname{Rim}}{8}$}} & \multirow{2}{*}{\multicolumn{2}{|c|}{ Neck }} & \multicolumn{2}{|c|}{ Carinated } & \multicolumn{2}{|c|}{ Narrow orifice } & \multirow{2}{*}{$\begin{array}{r}\text { Total } \\
77\end{array}$} & \multirow{2}{*}{$\frac{\text { Weight (g) }}{383}$} \\
\hline & & & 6 & & & & & & & & & & \\
\hline 190-200 & & & 7 & & 7 & & & & & & & 14 & 125 \\
\hline $200-210$ & 10 & & 36 & & 15 & & 2 & & 1 & & & 64 & 571 \\
\hline $210-220$ & 354 & & 57 & & 13 & & 11 & & 2 & & 1 & 438 & 2001 \\
\hline $220-230$ & 255 & & 111 & & 24 & & 4 & & 1 & & 4 & 399 & 3609 \\
\hline $230-240$ & 268 & & 89 & & 16 & & 7 & & 2 & & 2 & 384 & 3022 \\
\hline $240-250$ & 207 & & 83 & & 28 & & 3 & & 4 & & & 325 & 2452 \\
\hline $250-260$ & 119 & & 16 & & 10 & & 2 & & & & & 147 & 1330 \\
\hline $260-280$ & & & 4 & & & & & & & & & 4 & 9 \\
\hline Total & 1276 & & 09 & & 121 & & 29 & & 10 & & 7 & 1852 & 13493 \\
\hline Depth (cm) & CPI-X & CPI-// & CPI- & & & ssed & & tool & Appl & iqué & $\begin{array}{l}\text { Incised } \\
\text { symm. }\end{array}$ & $\begin{array}{l}\text { End-tool } \\
\text { impressed }\end{array}$ & $\begin{array}{l}\text { Finger } \\
\text { pinching }\end{array}$ \\
\hline 180-190 & 2 & & & & & & & 1 & & & & & \\
\hline 190-200 & 2 & 1 & & & & & & 1 & & & & 1 & \\
\hline 200-210 & 11 & 4 & & & & & & 2 & & 1 & & 8 & \\
\hline $210-220$ & 16 & 12 & & & & 2 & & 4 & & 3 & 3 & 3 & \\
\hline $220-230$ & 35 & 20 & & & & 1 & & 5 & & & 1 & 14 & \\
\hline $230-240$ & 34 & 14 & & & & 1 & & 5 & & & 2 & 7 & \\
\hline $240-250$ & 30 & 28 & & & & 1 & & 5 & & & & 9 & 1 \\
\hline $250-260$ & 3 & 8 & & & & & & 1 & & & & 1 & \\
\hline $260-280$ & 1 & & & & & & & & & & & & \\
\hline Total & 134 & 87 & & & & 5 & & 24 & & 4 & 6 & 43 & 1 \\
\hline
\end{tabular}


a dispersed and dimpled surface. Body sherds decorated by wavy impressions exhibit greater variability in pattern. They include chevrons (lines angled at $35-55^{\circ}$ ) and shallow ridges of uncertain form. Often the relief is faint or heavily superimposed and it is unclear whether a carved paddle was used to apply all of the surface modification classed as wavy relief. For example, the curvilinear shape on some sherds could have resulted from denting the pot with the edge of a carved paddle or other tool to create an uneven rippled surface. A wavy surface could also have been made with natural materials such as bark to impress wavy ridges or tool/ finger gouging followed by smoothing.

Form 1A jars: The majority of Layer 4 rims $(71 \%)$ are from 'jars'. The rims have straight or slightly curving rim courses that are parallel $(50 \%)$, slightly thickened $(29 \%)$ or gradually thinning towards the lip (16\%). Two kinds of lip form were identified - flat/flat-rounded (91\%) and a few rounded/pointed. Side-tool lip treatment was found on 16\% of Form $1 \mathrm{~A}$ rims and $3.5 \%$ were marked with an end tool. Rim eversion varies from $2^{\circ}$ to $32^{\circ}$ with a mean of $16^{\circ}$. The thickness of the rim course is $4.3-11.0 \mathrm{~mm}$ (mean $7.4 \mathrm{~mm}$ ), while the rim height is $17-56 \mathrm{~mm}$, with an average of $30 \mathrm{~mm}$. Inclination angles from $22 \mathrm{rim}$ sherds have a mean of $129^{\circ}$ (range $110-151^{\circ}$ ). Vessel diameters (41 sherds) are mostly in the 18-26 cm range (mean of $22 \mathrm{~cm}$ ), with a maximum recorded vessel diameter of $38 \mathrm{~cm}$ and a minimum of $12 \mathrm{~cm}$. Seven Form 1A jars were identified in the Layer 4 assemblage.

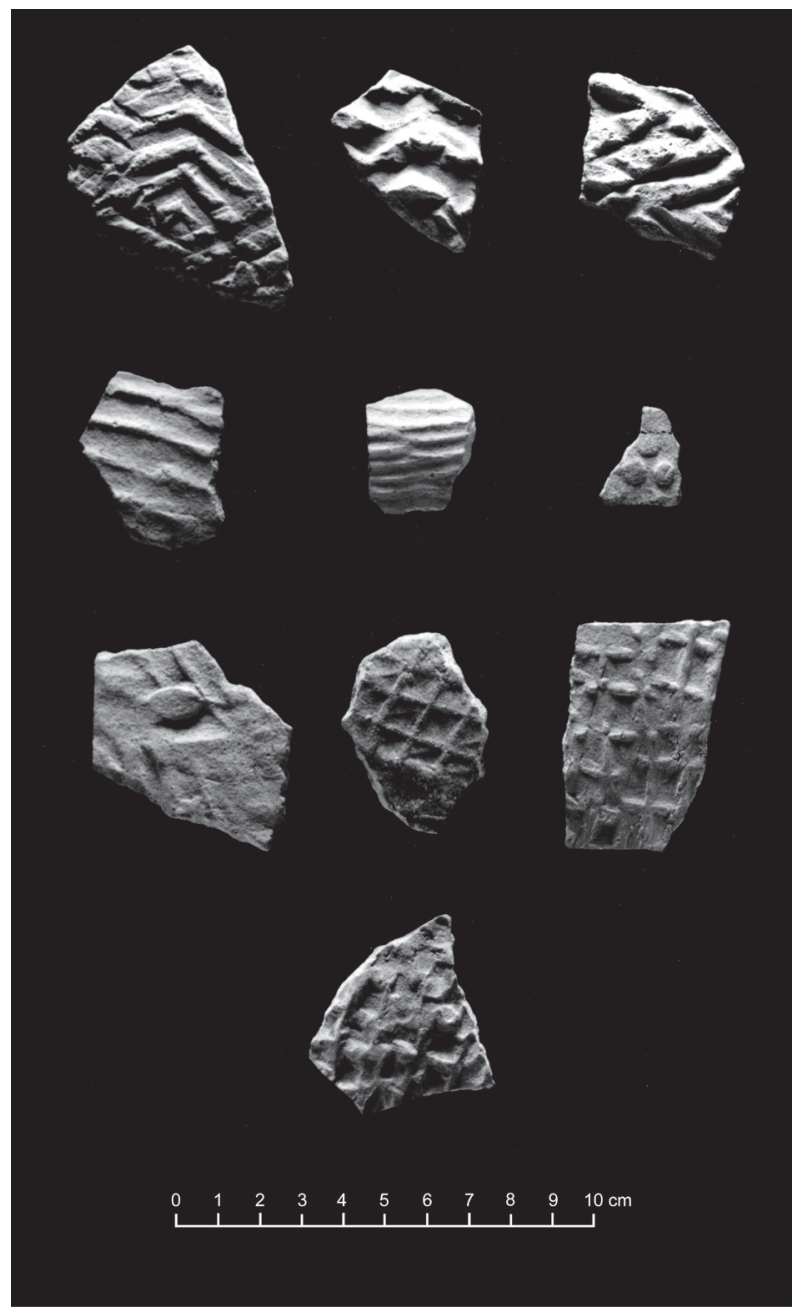

Figure 118. Navatu 17A, Layer 4 sherds decorated with curvilinear, parallel-rib, spot and cross-hatch relief.

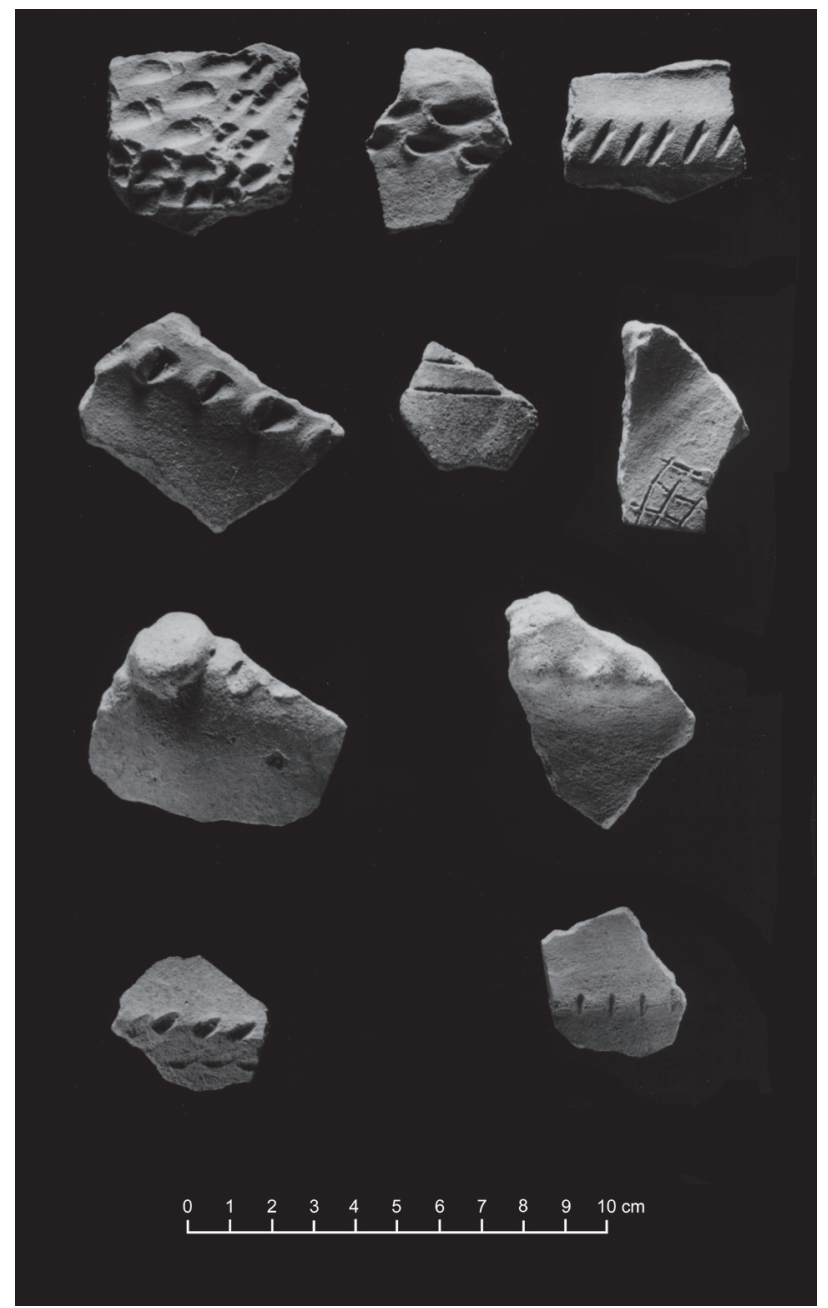

Figure 119. Navatu 17A, Layer 4 sherds decorated with end and side-tool impression, finger-gouging, incision and modelling. 
The diameter of these vessels is $17-29 \mathrm{~cm}$. Two of the vessels have notched lips and one has rows of oval end-tool impressions on the neck. Lips are flat/flat-rounded and the mean rim eversion is $24^{\circ}$. No paddle-relief markings were found on the rim or neck and few of these sherds from the Layer 4 assemblage have attached body-sherd portions. It is likely that some of these vessels had paddle relief, given the presence of paddle-relief vessels in Gifford's (1951) much larger sample.

\section{Form 2 bowls}

Form 2A everted rim/direct rim-body contour: A single Form 2A vessel, with parallel relief, a flat-rounded lip and a diameter of $20 \mathrm{~cm}$, was identified.

Form 2B inverted rim/direct rim-body contour: There are three vessels with rims inverted from $2^{\circ}$ to $43^{\circ}$. The vessel with the high inversion angle has an inturned lip and otherwise would have a much smaller degree of rim inversion. The lip forms are flat-rounded and two are marked with paddle relief (curvilinear and parallel rib).

Form $2 \mathrm{C}$ inverted rim/indirect body contour: Three Form 2C vessels have an inversion angle range of $38^{\circ}$ to $40^{\circ}$ and gradually thinning rims, terminating in pointed lips. Decoration consists of deep finger gouges/pinching on the circumference, diagonal incision and circular punctate on the rim. Their diameters are between $22 \mathrm{~cm}$ and $30 \mathrm{~cm}$. These vessels have finger impressions below the rim-body corner point and comprise a distinct vessel form at Navatu 17A.

\section{Form 4 narrow-orifice vessels}

Form 4A single orifice/indirect rim-body contour: The only single-orifice vessel has a neck height of $17 \mathrm{~mm}$ and internal orifice dimensions of $17 \mathrm{~mm} \times 21 \mathrm{~mm}$. It is decorated with oval end-tool impressions and appears to have had an ovoid or flask-shaped body.

Form 4C double orifice and stirrup handle: Two forms of double-spouted vessel were identified. The first has a stirrup handle, with an interior handle diameter of $17-31 \mathrm{~mm}$. The orifices exit the dorsal surface of the handle (internal orifice diameter $10-15 \mathrm{~mm}$ ). Spout openings are angled $0-10^{\circ}$. In the second form, the spouts are oriented horizontally, and it is possible that a small handle bridged the spouts. The internal spout diameter range is $7.6-13.0 \mathrm{~mm}$. The body of the double-orifice vessel appears to have been oval-toglobular, with the housing containing the vessel openings protruding above the body. The handle-orifice component is probably centered over the body. Decoration is limited to the application of applied nubbins around the circumference of the handle base and modelling of the stirrup handle (Gifford 1951:Plate 23jk).

Carinated vessels: The vessel forms associated with the carinated sherds are unclear. Sherd curvature and thickness suggests that carinated sherds came from small to mid-sized vessels. Shaw (1967:82) identified two carinated forms at Navatu 17A. The first belonged to an inverted bowl. Lips were described as rounded and bevelled and vessel size was similar to the ethnographically known vuluvulu or finger bowl. Carinated sherds from Layer 4 are commonly decorated below the carination line with paddle markings (parallel rib and curvilinear) and punctation, and on the carination line with end-tool impressions. Other carinated forms have everted indirect rims approximately $4 \mathrm{~cm}$ in length and flat-rounded lips. 


\section{Ugaga Island ceramics}

The Ugaga Island ceramic collection, from an excavated area of 55 sq. $\mathrm{m}$ (Test Pit 1 and U6 ceramics were not analysed), consists of 20,793 sherds weighing a total of $115.4 \mathrm{~kg}$ (Table 46). More than $80 \%$ of sherds (by number and weight) occurred between $10 \mathrm{~cm}$ and $40 \mathrm{~cm}$ depth. Plain body sherds comprise the majority of the assemblage, at $91.2 \%$, with smaller numbers of decorated body sherds (4.3\%), rims (3.8\%) and necks (0.6\%). Spouts, carinations, '?lugs', '?stands' and handles are present in small numbers $(0.1 \%)$ and the majority were found in the large excavation area. Eastern excavation squares had low-to-medium sherd numbers and weights, while squares in the main excavation area such as M6, O7-O8, O10 and P8, and $\mathrm{T} / \mathrm{U}-1$ in the west, had the largest sherd numbers.

The main types of surface modification found in the assemblage are shown in Table 47, along with the percentage of plain sherds. Plain sherds make up more than $90 \%$ of the ceramics in all but two squares. Squares I9 and I10 had large numbers of shell-impressed and incised sherds that derived from a single vessel (see below). Crosby (1988:125) recorded decorated sherd percentages at Ugaga of 40-55\%, but his small sample was not representative of the site. Sherds with surface modification were more abundant in the central excavation area than the smaller eastern areas

Sherds with dentate stamping were present in the main excavation area but not in the smaller areas to the east and west. The 91 sherds with dentate stamping ( $0.4 \%$ of the assemblage) were dispersed in rows $\mathrm{K}-\mathrm{M}$ and there was no evidence for concentrations of dentate sherds by square or area within the main excavation zone. Stratigraphically, the majority of dentate sherds occurred in the $20-30 \mathrm{~cm}(\mathrm{n}=25)$ and $30-40 \mathrm{~cm}(\mathrm{n}=46)$ spits. Fifteen sherds initially recorded as dentate stamped were later identified under low-power magnification as having a crenate edge consistent with stamping the edge of a shell valve edge in the clay vessel.

The majority of sherds with dentate stamping have single or double lines of impressions. Recognisable design elements and motifs (e.g. Anson 1983; Best 1984) are shown in Figures 120 and 121. The limited number of motifs and the 'exploded' or open dentate-stamped patterns have a parallel in eastern Lapita assemblages (Sigatoka, Mulifanua and Niuatoputapu), as well as in what Summerhayes (1996) termed 'Late Lapita' assemblages from Near and Remote Oceania.

At Ugaga, dentate stamping is associated with appliqué fillet, symmetric incision, shell impression and a red slip. It is probable that many of the dentate-stamped sherds were red slipped, but the delicate layer of soluble iron oxide was subject to chemical and physical weathering and has not survived in the shallow and disturbed Ugaga deposits. The dentate tools are variable in width, number of teeth and amount of wear. For example, on some sherds the corners of the tooth surface were irregularly rounded, producing uneven oval or circular punctations. In contrast, the tool used to decorate several other vessels was sharp edged, with almost twice the number of teeth per centimetre.

Fifteen rims and four carinations are dentate stamped. The rims are from Form 1 vessels with flaring rims. Dentate stamping is commonly applied to the lip surface and 11 rims have transverse rows of stamping (2-3 teeth). Dentate stamping of interior and exterior rim surfaces is often associated with lip stamping.

Sherds with fine striations make up $0.3 \%$ of the assemblage. They occurred in the eastern, central and western excavation areas and $73 \%$ of them were found at $20-40 \mathrm{~cm}$ depth. Wiping as a form of surface modification is variable in the Ugaga assemblage with some sherds showing faint, and others deep, striations. However, the striations were always applied horizontally and they are almost exclusively on the rim and neck. It seems likely that wipe marks were produced 
Table 46. Ugaga Island ceramics: Sherd type by excavation square and depth.

\begin{tabular}{|c|c|c|c|c|c|c|c|}
\hline Square/ depth (cm) & Plain body & Decorated body & Rims & Necks & Other & Total & Weight (g) \\
\hline \multicolumn{8}{|l|}{$-A 13$} \\
\hline $0-10$ & 35 & & 2 & 1 & & 38 & 195.6 \\
\hline $10-20$ & 37 & 2 & 1 & & & 40 & 269.7 \\
\hline $20-30$ & 34 & & 2 & & & 36 & 229.9 \\
\hline $30-40$ & 11 & & & & & 11 & 38.8 \\
\hline Total & & & & & & 125 & 734 \\
\hline \multicolumn{8}{|l|}{-A14 } \\
\hline $0-10$ & 79 & & 1 & 1 & & 81 & 306.3 \\
\hline $10-20$ & 43 & & & & & 43 & 223.7 \\
\hline $20-30$ & 37 & 1 & 2 & & & 40 & 164.7 \\
\hline $30-40$ & 44 & 4 & 3 & 1 & & 52 & 322.2 \\
\hline Total & & & & & & 216 & 1016.7 \\
\hline \multicolumn{8}{|l|}{-A15 } \\
\hline $0-10$ & 23 & & 1 & & & 24 & 169.4 \\
\hline $10-20$ & 56 & 2 & 4 & 1 & & 63 & 423.6 \\
\hline $20-30$ & 30 & & 1 & & & 31 & 102.3 \\
\hline $30-40$ & 41 & & 1 & & & 42 & 164.0 \\
\hline Total & & & & & & 160 & 859.3 \\
\hline \multicolumn{8}{|l|}{ C8 } \\
\hline $0-10$ & 58 & 2 & & 1 & & 61 & 335.3 \\
\hline $10-20$ & 123 & 2 & 2 & 2 & & 129 & 740.7 \\
\hline $20-30$ & 94 & & 2 & 3 & & 99 & 584.9 \\
\hline $30-40$ & 134 & 5 & 12 & 6 & & 157 & 787.0 \\
\hline $40-50$ & 1 & & & & & 1 & 22.0 \\
\hline 50-60 & & & 1 & & & 1 & 3.6 \\
\hline Total & & & & & & 448 & 2473.5 \\
\hline \multicolumn{8}{|l|}{ C9 } \\
\hline $0-10$ & 34 & 1 & & & & 25 & 175.4 \\
\hline $10-20$ & 120 & 4 & 5 & 1 & & 130 & 710.6 \\
\hline 20-30 & 224 & 18 & 6 & 2 & & 224 & 1279.5 \\
\hline 30-40 & 64 & 6 & 3 & 3 & & 76 & 548.6 \\
\hline Total & & & & & & 455 & 2714.1 \\
\hline \multicolumn{8}{|l|}{ C12 } \\
\hline $0-10$ & 30 & & & & & 30 & 94.1 \\
\hline $10-20$ & 74 & 2 & 3 & 3 & & 82 & 542.8 \\
\hline $20-30$ & 52 & 5 & 2 & 2 & & 61 & 301.6 \\
\hline $30-40$ & 88 & 8 & 8 & & 1 handle & 105 & 584.5 \\
\hline $40-50$ & 63 & 3 & 4 & 3 & & 73 & 320.3 \\
\hline Total & & & & & & 351 & 1843.3 \\
\hline \multicolumn{8}{|l|}{ C13 } \\
\hline $0-10$ & 57 & 2 & & & & 61 & 298.7 \\
\hline $10-20$ & 117 & 2 & 2 & 1 & & 129 & 545.0 \\
\hline $20-30$ & 131 & & 2 & 2 & & 99 & 846.9 \\
\hline $30-40$ & 81 & 5 & 12 & 3 & & 157 & 323.9 \\
\hline $40-50$ & 21 & & & 6 & & 1 & 146.8 \\
\hline \multicolumn{8}{|l|}{ 50-60 } \\
\hline Total & & & & & & 447 & 2161.3 \\
\hline
\end{tabular}


270 Geoffrey Clark

Table 46 continued

\begin{tabular}{|c|c|c|c|c|c|c|c|}
\hline Square/ depth (cm) & Plain body & Decorated body & Rims & Necks & Other & Total & Weight (g) \\
\hline \multicolumn{8}{|l|}{ D12 } \\
\hline 0-10 & 22 & & & & & 22 & 134.4 \\
\hline $10-20$ & 80 & & 3 & & & 83 & 541.7 \\
\hline $20-30$ & 74 & 2 & 1 & 1 & 1 ?stand & 79 & 447.0 \\
\hline $30-40$ & 70 & 6 & 9 & & & 85 & 528.7 \\
\hline $40-50$ & 82 & & & 2 & & 84 & 323.7 \\
\hline Total & & & & & & 353 & 1975.5 \\
\hline \multicolumn{8}{|l|}{ D13 } \\
\hline $0-10$ & 54 & & 1 & 1 & & 56 & 235.2 \\
\hline $10-20$ & 102 & & 5 & 3 & & 110 & 737.4 \\
\hline $20-30$ & 123 & 4 & & 1 & & 128 & 553.1 \\
\hline $30-40$ & 74 & 1 & 5 & & & 80 & 426.7 \\
\hline $40-50$ & 52 & & & & & 52 & 201.1 \\
\hline $50-60$ & 23 & 2 & 2 & & & 27 & 96.7 \\
\hline Total & & & & & & 453 & 2250.2 \\
\hline \multicolumn{8}{|l|}{ F8 } \\
\hline $0-10$ & 14 & & & & & 14 & 37.2 \\
\hline $10-20$ & 78 & 3 & 6 & & & 87 & 494.5 \\
\hline $20-30$ & 72 & 6 & 4 & 3 & & 85 & 554.5 \\
\hline $30-40$ & 62 & 9 & 10 & 1 & & 82 & 639.6 \\
\hline $40-50$ & 81 & 2 & & & & 83 & 257.5 \\
\hline $50-60$ & 49 & & 2 & 3 & & 54 & 189.9 \\
\hline $60-70$ & 28 & 3 & & & & 31 & 131.4 \\
\hline Total & & & & & & 436 & 2304.6 \\
\hline \multicolumn{8}{|l|}{ G8 } \\
\hline $0-10$ & 20 & & & & & 20 & 47.8 \\
\hline $10-20$ & 100 & 3 & 4 & 1 & & 108 & 379.2 \\
\hline $20-30$ & 112 & 15 & 2 & & & 129 & 820.6 \\
\hline $30-40$ & 94 & & 9 & 1 & & 104 & 646.4 \\
\hline $40-50$ & 64 & 7 & 1 & 1 & & 73 & 319.3 \\
\hline Total & & & & & & 434 & 2213.3 \\
\hline \multicolumn{8}{|l|}{ H8 } \\
\hline $0-10$ & 8 & & & & & 8 & 25.6 \\
\hline $10-20$ & 53 & 3 & 1 & & & 57 & 263.4 \\
\hline $20-30$ & 87 & 4 & & 2 & & 93 & 657.6 \\
\hline $30-40$ & 69 & 3 & 3 & 1 & & 76 & 421.9 \\
\hline $40-50$ & 65 & & 4 & & & 69 & 192.2 \\
\hline Total & & & & & & 303 & 1560.7 \\
\hline \multicolumn{8}{|l|}{15} \\
\hline $0-10$ & 26 & & & & & 26 & 104.9 \\
\hline $10-20$ & 55 & & 1 & 2 & & 58 & 312.9 \\
\hline $20-30$ & 60 & 1 & 3 & 1 & & 65 & 403.4 \\
\hline $30-40$ & 177 & 7 & 6 & & & 190 & 1690.0 \\
\hline $40-50$ & 46 & & 4 & 1 & & 51 & 285.4 \\
\hline Total & & & & & & 390 & 2796.6 \\
\hline
\end{tabular}


Table 46 continued

\begin{tabular}{|c|c|c|c|c|c|c|c|}
\hline Square/ depth (cm) & Plain body & Decorated body & Rims & Necks & Other & Total & Weight (g) \\
\hline \multicolumn{8}{|l|}{16} \\
\hline $0-10$ & 25 & 3 & 1 & & & 29 & 140.7 \\
\hline $10-20$ & 38 & 3 & 2 & & & 43 & 382.0 \\
\hline $20-30$ & 137 & & 6 & & 1?stand & 144 & 966.7 \\
\hline $30-40$ & 74 & & 4 & & & 79 & 565.2 \\
\hline $40-50$ & 60 & & 5 & & & 65 & 255.1 \\
\hline Total & & & & & & 360 & 2309.7 \\
\hline \multicolumn{8}{|l|}{17} \\
\hline $0-10$ & 3 & & 2 & & & 5 & 343.9 \\
\hline $10-20$ & 94 & 3 & 8 & & & 105 & 616.0 \\
\hline $20-30$ & 104 & 2 & 5 & 2 & & 113 & 400.0 \\
\hline $30-40$ & 37 & & 2 & & & 39 & 78.0 \\
\hline \multicolumn{8}{|l|}{$40-50$} \\
\hline Total & & & & & & 262 & 1437.9 \\
\hline \multicolumn{8}{|l|}{18} \\
\hline $0-10$ & 41 & 3 & & & & 44 & 181.5 \\
\hline $10-20$ & 8 & & & & & 8 & 70.1 \\
\hline $20-30$ & 60 & 3 & 1 & 3 & & 67 & 298.9 \\
\hline $30-40$ & 151 & 8 & 4 & 1 & & 164 & 755.3 \\
\hline $40-50$ & 57 & 1 & 3 & & & 61 & 236.7 \\
\hline $50-60$ & 23 & 1 & 4 & & & 28 & 141.7 \\
\hline $60-70$ & 7 & & & & & 7 & 10.5 \\
\hline Total & & & & & & 379 & 1694.7 \\
\hline \multicolumn{8}{|l|}{19} \\
\hline $0-10$ & 22 & 2 & 1 & & & 25 & 106.4 \\
\hline $10-20$ & 18 & 14 & & & & 32 & 152.9 \\
\hline $20-30$ & 69 & 2 & & & & 71 & 312.2 \\
\hline $30-40$ & 103 & 5 & 4 & & 1 handle & 113 & 679.3 \\
\hline $40-50$ & 36 & & 2 & & & 38 & 213.9 \\
\hline Total & & & & & & 279 & 1464.7 \\
\hline \multicolumn{8}{|l|}{110} \\
\hline $0-10$ & 40 & 1 & & & & 41 & 172.7 \\
\hline $10-20$ & 107 & 18 & 3 & & & 128 & 632.4 \\
\hline $20-30$ & 118 & 5 & 5 & 1 & & 129 & 777.9 \\
\hline $30-40$ & 64 & & 3 & & & 67 & 294.7 \\
\hline $40-50$ & 51 & & 2 & & & 53 & 178.4 \\
\hline Total & & & & & & 418 & 2056.1 \\
\hline \multicolumn{8}{|l|}{$J 5$} \\
\hline $0-10$ & 29 & 2 & 1 & 1 & & 33 & 234.7 \\
\hline $10-20$ & 42 & 10 & & 2 & & 54 & 401.1 \\
\hline $20-30$ & 56 & 5 & 7 & 1 & & 69 & 550.7 \\
\hline $30-40$ & 125 & 4 & 3 & 1 & & 133 & 1371.5 \\
\hline $40-50$ & 77 & 3 & 4 & 2 & & 86 & 670.7 \\
\hline Total & & & & & & 365 & 3228.7 \\
\hline
\end{tabular}


272 Geoffrey Clark

Table 46 continued

\begin{tabular}{|c|c|c|c|c|c|c|c|}
\hline Square/ depth (cm) & Plain body & Decorated body & Rims & Necks & Other & Total & Weight (g) \\
\hline \multicolumn{8}{|l|}{ J6 } \\
\hline $0-10$ & 21 & 1 & & & & 22 & 201.1 \\
\hline $10-20$ & 26 & & & & & 26 & 243.7 \\
\hline $20-30$ & 55 & 1 & 3 & 2 & & 61 & 476.9 \\
\hline $30-40$ & 103 & 2 & 7 & 1 & & 113 & 1107.5 \\
\hline $40-50$ & 72 & 1 & 4 & & & 77 & 430.1 \\
\hline Total & & & & & & 299 & 2459.3 \\
\hline \multicolumn{8}{|l|}{ J7 } \\
\hline $0-10$ & 21 & & 1 & & & 22 & 106.8 \\
\hline $10-20$ & 51 & 3 & & & & 54 & 325.6 \\
\hline $20-30$ & 72 & 6 & 3 & & & 81 & 392.9 \\
\hline $30-40$ & 142 & 3 & 8 & & 1 handle & 154 & 1037.7 \\
\hline $40-50$ & 43 & & 3 & 1 & & 47 & 221.3 \\
\hline Total & & & & & & 358 & 2084.3 \\
\hline \multicolumn{8}{|l|}{$J 8$} \\
\hline $0-10$ & 16 & 1 & & & & 17 & 174.9 \\
\hline $10-20$ & 30 & & & & & 30 & 163.2 \\
\hline $20-30$ & 39 & 6 & 6 & & & 51 & 479 \\
\hline $30-40$ & 86 & 4 & 13 & & & 103 & 780.6 \\
\hline $40-50$ & 38 & 2 & 7 & & & 47 & 256 \\
\hline Total & & & & & & 248 & 1853.7 \\
\hline \multicolumn{8}{|l|}{ J9 } \\
\hline $0-10$ & 40 & & 1 & & & 41 & 48.4 \\
\hline $10-20$ & 77 & & 1 & & & 78 & 291.6 \\
\hline $20-30$ & 99 & 8 & 2 & 1 & 2?lug & 112 & 666.4 \\
\hline $30-40$ & 98 & 6 & 3 & & & 107 & 452 \\
\hline $40-50$ & 17 & & 4 & & & 21 & 132.4 \\
\hline Total & & & & & & 359 & 1590.8 \\
\hline \multicolumn{8}{|l|}{$\mathrm{J} 10$} \\
\hline $0-10$ & 4 & & & & & 4 & 13.1 \\
\hline $10-20$ & 70 & 3 & & & & 73 & 430.6 \\
\hline $20-30$ & 70 & 6 & 6 & 2 & & 84 & 575.1 \\
\hline $30-40$ & 103 & 16 & 3 & & & 122 & 667.5 \\
\hline $40-50$ & 36 & 1 & 2 & & & 39 & 231.0 \\
\hline Total & & & & & & 322 & 1917.3 \\
\hline \multicolumn{8}{|l|}{ K5 } \\
\hline $0-10$ & 25 & & 1 & & & 26 & 108.9 \\
\hline $10-20$ & 62 & 4 & 3 & & & 69 & 357 \\
\hline $20-30$ & 46 & 5 & 2 & & & 53 & 316 \\
\hline $30-40$ & 116 & 9 & 4 & & carination & 129 & 1007.8 \\
\hline $40-50$ & 57 & & 4 & & carination & 63 & 264.4 \\
\hline Total & & & & & & 340 & 2054.1 \\
\hline \multicolumn{8}{|l|}{ K6 } \\
\hline $0-10$ & 34 & & & & & 34 & 113.7 \\
\hline $10-20$ & 56 & 1 & & & & 57 & 222 \\
\hline $20-30$ & 132 & 5 & 2 & & & 139 & 648.4 \\
\hline $30-40$ & 108 & 6 & 4 & & & 118 & 550.3 \\
\hline $40-50$ & 73 & 8 & 6 & & & 87 & 462.3 \\
\hline Total & & & & & & 435 & 1996.7 \\
\hline
\end{tabular}


Table 46 continued

\begin{tabular}{|c|c|c|c|c|c|c|c|}
\hline Square/ depth (cm) & Plain body & Decorated body & Rims & Necks & Other & Total & Weight (g) \\
\hline \multicolumn{8}{|l|}{ K7 } \\
\hline $0-10$ & 25 & & & & & 25 & 102.7 \\
\hline $10-20$ & 31 & & & & & 31 & 190.6 \\
\hline $20-30$ & 39 & & & & & 39 & 222.6 \\
\hline $30-40$ & 141 & 2 & 7 & 1 & 1 ?lug & 152 & 767.6 \\
\hline $40-50$ & 51 & 4 & & & & 55 & 247.1 \\
\hline Total & & & & & & 302 & 1530.6 \\
\hline \multicolumn{8}{|l|}{ K8 } \\
\hline $0-10$ & 18 & 1 & & & & 19 & 97.8 \\
\hline $10-20$ & 42 & 5 & & & & 47 & 242 \\
\hline $20-30$ & 33 & 11 & 3 & & & 47 & 441.1 \\
\hline $30-40$ & 61 & & 6 & & & 67 & 465.8 \\
\hline $40-50$ & 56 & & & & & 56 & 146.1 \\
\hline Total & & & & & & 236 & 1392.8 \\
\hline \multicolumn{8}{|l|}{ K9 } \\
\hline $0-10$ & 39 & & & & & 39 & 162.9 \\
\hline $10-20$ & 105 & 3 & 2 & & & 110 & 570.2 \\
\hline $20-30$ & 69 & 3 & 1 & 1 & & 74 & 426.5 \\
\hline $30-40$ & 78 & 4 & 2 & & carination & 85 & 541.7 \\
\hline $40-50$ & 58 & 1 & 1 & & & 60 & 296.3 \\
\hline Total & & & & & & 368 & 1997.6 \\
\hline \multicolumn{8}{|l|}{ K10 } \\
\hline $0-10$ & 27 & & & & & 27 & 82.8 \\
\hline $10-20$ & 84 & 2 & 7 & & & 93 & 445.9 \\
\hline $20-30$ & 85 & 2 & & 2 & & 90 & 391.9 \\
\hline $30-40$ & 110 & 9 & 7 & & 1 handle & 126 & 610.6 \\
\hline $40-50$ & 68 & 1 & 1 & & & 70 & 182.4 \\
\hline Total & & & & & & 406 & 1713.6 \\
\hline \multicolumn{8}{|l|}{ L5 } \\
\hline $0-10$ & 39 & 3 & 2 & & & 44 & 254.2 \\
\hline $10-20$ & 99 & 5 & 4 & 1 & & 109 & 530.3 \\
\hline $20-30$ & 140 & 15 & 5 & 2 & & 162 & 940.7 \\
\hline $30-40$ & 171 & 13 & 11 & 1 & 1 ?stand & 197 & 1117.2 \\
\hline Total & & & & & & 512 & 2842.4 \\
\hline \multicolumn{8}{|l|}{ L6 } \\
\hline $0-10$ & 12 & & & & & 12 & 42.9 \\
\hline $10-20$ & 39 & & 4 & 1 & & 44 & 302.9 \\
\hline $20-30$ & 103 & 12 & 4 & & & 119 & 758.6 \\
\hline $30-40$ & 95 & & 10 & 1 & 1 handle & 107 & 503.7 \\
\hline $40-50$ & 26 & 4 & 3 & & & 33 & 160.9 \\
\hline Total & & & & & & 315 & 1769 \\
\hline \multicolumn{8}{|l|}{ L7 } \\
\hline $0-10$ & 22 & & 1 & & & 23 & 83 \\
\hline $10-20$ & 47 & & & & & 47 & 180.9 \\
\hline $20-30$ & 25 & 1 & 4 & & 1 handle & 31 & 336.7 \\
\hline $30-40$ & 88 & 12 & 9 & & & 109 & 989.5 \\
\hline $40-50$ & 49 & & 3 & & & 52 & 169.4 \\
\hline Total & & & & & & 262 & 1759.5 \\
\hline
\end{tabular}


274 Geoffrey Clark

Table 46 continued

\begin{tabular}{|c|c|c|c|c|c|c|c|}
\hline Square/ depth (cm) & Plain body & Decorated body & Rims & Necks & Other & Total & Weight (g) \\
\hline \multicolumn{8}{|l|}{ L8 } \\
\hline $0-10$ & 5 & 1 & & & & 6 & 35.3 \\
\hline $10-20$ & 20 & & & & & 20 & 66.4 \\
\hline $20-30$ & 62 & & 1 & & & 63 & 372 \\
\hline $30-40$ & 71 & 6 & 7 & & 1?lug & 86 & 671.8 \\
\hline $40-50$ & 48 & & 8 & & & 56 & 465.4 \\
\hline $50-60$ & 9 & 1 & & & & 10 & 19.7 \\
\hline Total & & & & & & 241 & 1630.6 \\
\hline \multicolumn{8}{|c|}{ L9 } \\
\hline $0-10$ & 17 & 1 & & & & 18 & 60.3 \\
\hline $10-20$ & 96 & 4 & 3 & & & 103 & 299.6 \\
\hline $20-30$ & 149 & 8 & & & & 157 & 658.6 \\
\hline $30-40$ & 7 & 6 & 6 & & & 19 & 153.9 \\
\hline $40-50$ & 21 & 1 & 1 & & & 23 & 178.6 \\
\hline Total & & & & & & 320 & 1351 \\
\hline \multicolumn{8}{|l|}{ L10 } \\
\hline $0-10$ & 100 & 3 & 1 & & & 104 & 508.5 \\
\hline $10-20$ & 74 & 2 & & & & 76 & 357.6 \\
\hline $20-30$ & 83 & 4 & 3 & & & 90 & 538.4 \\
\hline $30-40$ & 73 & 4 & 2 & & & 79 & 417.1 \\
\hline $40-50$ & 25 & & 2 & & & 27 & 96.6 \\
\hline Total & & & & & & 376 & 1918.2 \\
\hline \multicolumn{8}{|l|}{ M5 } \\
\hline $0-10$ & 40 & 2 & 1 & 1 & & 44 & 150.5 \\
\hline $10-20$ & 92 & 7 & 6 & & & 105 & 316.9 \\
\hline $20-30$ & 151 & 17 & 7 & 2 & & 177 & 876.7 \\
\hline $30-40$ & 138 & & & & carination & 139 & 583.2 \\
\hline $40-50$ & 62 & 1 & & & carination & 64 & 206.3 \\
\hline Total & & & & & & 424 & 2133.6 \\
\hline \multicolumn{8}{|l|}{ M6 } \\
\hline $0-10$ & 26 & & 1 & & & 27 & 86.5 \\
\hline $10-20$ & 110 & 8 & & 1 & & 119 & 366.3 \\
\hline $20-30$ & 153 & 19 & 7 & 2 & carination & 182 & 1495.6 \\
\hline $30-40$ & 137 & 5 & 6 & 1 & & 149 & 875.6 \\
\hline $40-50$ & 40 & 3 & & & & 43 & 222.2 \\
\hline Total & & & & & & 520 & 3046.2 \\
\hline \multicolumn{8}{|l|}{$\overline{\text { M7 }}$} \\
\hline $0-10$ & 27 & 4 & & & & 31 & 157.9 \\
\hline $10-20$ & 89 & 5 & 1 & & & 95 & 290.3 \\
\hline $20-30$ & 110 & 4 & 2 & & & 116 & 638.5 \\
\hline $30-40$ & 52 & 1 & 7 & 1 & & 61 & 472 \\
\hline $40-50$ & 32 & & 2 & & & 34 & 192.9 \\
\hline Total & & & & & & 337 & 1751.6 \\
\hline \multicolumn{8}{|l|}{$\overline{M 8}$} \\
\hline $0-10$ & 16 & 2 & & & & 18 & 100.3 \\
\hline $10-20$ & 37 & 1 & & & & 38 & 277.4 \\
\hline $20-30$ & 72 & 8 & 1 & & & 81 & 378.1 \\
\hline $30-40$ & 124 & 2 & 2 & & 1 ?stand & 129 & 599.5 \\
\hline $40-50$ & 41 & 1 & & 1 & & 43 & 264.1 \\
\hline \multicolumn{8}{|l|}{$50-60$} \\
\hline Total & & & & & & 309 & 1619.4 \\
\hline
\end{tabular}


Table 46 continued

\begin{tabular}{|c|c|c|c|c|c|c|c|}
\hline Square/ depth (cm) & Plain body & Decorated body & Rims & Necks & 0ther & Total & Weight (g) \\
\hline \multicolumn{8}{|l|}{ M9 } \\
\hline $0-10$ & 20 & & & & & 20 & 92.7 \\
\hline $10-20$ & 121 & 6 & 4 & & & 131 & 665.3 \\
\hline $20-30$ & 128 & 7 & 2 & & & 137 & 599.4 \\
\hline $30-40$ & 134 & 1 & 9 & & carination & 145 & 888.4 \\
\hline $40-50$ & 37 & 1 & 3 & & & 41 & 203.1 \\
\hline Total & & & & & & 474 & 2448.9 \\
\hline \multicolumn{8}{|l|}{ M10 } \\
\hline $0-10$ & 74 & 1 & & & & 75 & 324.8 \\
\hline $10-20$ & 119 & 1 & 5 & & & 126 & 374.4 \\
\hline $20-30$ & 106 & 7 & 3 & & 1 handle & 117 & 499.8 \\
\hline $30-40$ & 232 & 7 & 10 & & & 249 & 936.2 \\
\hline $40-50$ & 24 & & & & & 24 & 56.1 \\
\hline Total & & & & & & 591 & 2191.3 \\
\hline \multicolumn{8}{|l|}{ N8 } \\
\hline $0-10$ & 11 & & & & & 11 & 60.2 \\
\hline $10-20$ & 75 & 4 & 3 & 1 & & 83 & 479.8 \\
\hline $20-30$ & 92 & 9 & 3 & & & 104 & 677.9 \\
\hline $30-40$ & 90 & 3 & 11 & 1 & carination & 106 & 614.3 \\
\hline $40-50$ & 56 & 2 & 2 & & & 60 & 395.2 \\
\hline $50-60$ & 1 & & & & & 1 & 17.1 \\
\hline Total & & & & & & 365 & 2244.5 \\
\hline \multicolumn{8}{|l|}{ N9 } \\
\hline $0-10$ & 112 & & 5 & & & 117 & 552.2 \\
\hline $10-20$ & 132 & 4 & 7 & & & 143 & 937.4 \\
\hline $20-30$ & 79 & 12 & 5 & 1 & carination & 98 & 621.1 \\
\hline $30-40$ & 129 & 4 & 6 & 1 & & 140 & 755.3 \\
\hline $40-50$ & 18 & 5 & 2 & & & 25 & 76 \\
\hline Total & & & & & & 523 & 2942 \\
\hline \multicolumn{8}{|l|}{ N10 } \\
\hline $0-10$ & 68 & 1 & 1 & 1 & & 71 & 590.3 \\
\hline $10-20$ & 155 & 3 & 2 & & & 160 & 727.2 \\
\hline $20-30$ & 92 & 12 & 9 & & & 113 & 820.9 \\
\hline $30-40$ & 147 & 1 & 6 & & & 154 & 670.4 \\
\hline $40-50$ & 10 & 1 & & & & 11 & 61.6 \\
\hline Total & & & & & & 509 & 2870.4 \\
\hline \multicolumn{8}{|l|}{08} \\
\hline $0-10$ & 49 & 2 & & & & 51 & 233.2 \\
\hline $10-20$ & 118 & 4 & 3 & & & 125 & 722 \\
\hline $20-30$ & 220 & 14 & 13 & & & 247 & 1581.3 \\
\hline $30-40$ & 156 & 12 & 15 & & & 183 & 1276 \\
\hline $40-50$ & 30 & 2 & 2 & & & 34 & 337.5 \\
\hline $50-60$ & & & & & & 5 & 50.6 \\
\hline Total & & & & & & 645 & 4200.6 \\
\hline \multicolumn{8}{|l|}{09} \\
\hline $0-10$ & 46 & 1 & 1 & & & 48 & 241.9 \\
\hline \multicolumn{8}{|l|}{$10-20$} \\
\hline $20-30$ & 102 & 9 & 6 & & & 117 & 559.5 \\
\hline $30-40$ & 56 & 4 & 11 & & & 71 & 556.3 \\
\hline $40-50$ & 4 & & 2 & & & 6 & 14.5 \\
\hline Total & & & & & & 242 & 1372.2 \\
\hline
\end{tabular}


276 Geoffrey Clark

Table 46 continued

\begin{tabular}{|c|c|c|c|c|c|c|c|}
\hline Square/ depth (cm) & Plain body & Decorated body & Rims & Necks & Other & Total & Weight (g) \\
\hline \multicolumn{8}{|l|}{010} \\
\hline $0-10$ & 142 & 6 & 6 & & & 154 & 696.5 \\
\hline $10-20$ & 205 & 22 & 5 & & & 232 & 1236.3 \\
\hline $20-30$ & 109 & 4 & 3 & & & 116 & 569.7 \\
\hline $30-40$ & 58 & 1 & & & & 59 & 280.1 \\
\hline $40-50$ & 3 & & & & & 3 & 15.3 \\
\hline Total & & & & & & 564 & 2797.9 \\
\hline \multicolumn{8}{|l|}{ P8 } \\
\hline $0-10$ & 24 & & & & & 24 & 177.4 \\
\hline $10-20$ & 47 & & & & & 57 & 332.3 \\
\hline $20-30$ & 138 & & 10 & & 1 ?lug & 149 & 976.5 \\
\hline $30-40$ & 176 & & 7 & & 1 ?lug & 184 & 967.9 \\
\hline $40-50$ & 28 & & & & & 28 & 122.4 \\
\hline Total & & & & & & 442 & 2576.5 \\
\hline \multicolumn{8}{|l|}{$\overline{P 9}$} \\
\hline $0-10$ & 110 & & 2 & & & 112 & 466.8 \\
\hline $10-20$ & 213 & 2 & 3 & & & 218 & 900.8 \\
\hline $20-30$ & 145 & 8 & 5 & 1 & & 159 & 1363.0 \\
\hline $30-40$ & 9 & 10 & 2 & & & 21 & 47.6 \\
\hline $40-50$ & 8 & & & & & 8 & 32.5 \\
\hline Total & & & & & & 518 & 2810.7 \\
\hline \multicolumn{8}{|l|}{ P10 } \\
\hline $0-10$ & 81 & 2 & 1 & & & 84 & 373.1 \\
\hline $10-20$ & 108 & 6 & 2 & & & 116 & 549.5 \\
\hline $20-30$ & 92 & 5 & 5 & & & 102 & 753.5 \\
\hline $30-40$ & 46 & 1 & 3 & & & 50 & 306.9 \\
\hline $40-50$ & 6 & & 1 & & & 7 & 49.0 \\
\hline Total & & & & & & 359 & 2031.8 \\
\hline \multicolumn{8}{|l|}{ Q10 } \\
\hline $0-10$ & 102 & & & & & 102 & 413.3 \\
\hline $10-20$ & 82 & 5 & 8 & & & 95 & 514.9 \\
\hline $20-30$ & 87 & 2 & 2 & & & 91 & 492 \\
\hline $30-40$ & 40 & & & & & 40 & 185.9 \\
\hline Total & & & & & & 328 & 1606.1 \\
\hline \multicolumn{8}{|l|}{ Q13 } \\
\hline $0-10$ & 9 & 2 & & & & 10 & 46.4 \\
\hline $10-20$ & 8 & & & & & 9 & 43 \\
\hline \multicolumn{8}{|l|}{$20-30$} \\
\hline $30-40$ & 90 & 3 & 5 & & & 98 & 443.9 \\
\hline Total & & & & & & 117 & 533.3 \\
\hline \multicolumn{8}{|l|}{$T / U-1$} \\
\hline $0-10$ & 40 & 6 & 1 & & & 47 & 325.5 \\
\hline $10-20$ & 47 & 8 & 9 & & 2 handles & 66 & 713.6 \\
\hline $20-30$ & 442 & 32 & 18 & 10 & & 502 & 3214.6 \\
\hline $30-40$ & 357 & 3 & 10 & & carination & 371 & 2217 \\
\hline $40-50$ & 74 & & & & & 74 & 480.1 \\
\hline $50-60$ & 83 & & 1 & & & 84 & 320.9 \\
\hline Total & & & & & & 1144 & 7271.7 \\
\hline
\end{tabular}


Table 47. Ugaga Island ceramics: Sherd decoration by excavation square and depth.

\begin{tabular}{|c|c|c|c|c|c|c|c|c|c|c|}
\hline Square/depth (cm) & Plain sherds & Dentate stamped & Wiped & Side tool & Shell imp. & Dents & $\mathrm{CPI}-/ /$ & CPI-X & Incision & End-tool imp. \\
\hline \multicolumn{11}{|l|}{$-A 13$} \\
\hline $0-10$ & 38 & & & 1 & & & & & & \\
\hline $10-20$ & 40 & & & & & & & & & \\
\hline $20-30$ & 36 & & & & & & & & & \\
\hline $30-40$ & 11 & & & & & & & & & \\
\hline \%Plain & 98.4 & & & & & & & & & \\
\hline \multicolumn{11}{|l|}{-A14 } \\
\hline $0-10$ & 81 & & & & & & & & & \\
\hline $10-20$ & 43 & & & & & & & & & \\
\hline $20-30$ & 39 & & & & & & & 1 & & \\
\hline $30-40$ & 49 & & & & & 1 & 1 & 1 & & \\
\hline \%Plain & 97.6 & & & & & & & & & \\
\hline \multicolumn{11}{|l|}{-A15 } \\
\hline $0-10$ & 24 & & & & & & & & & \\
\hline $10-20$ & 62 & & & 1 & & & 1 & & & \\
\hline $20-30$ & 31 & & & & & & & & & \\
\hline $30-40$ & 42 & & & & & & & & & \\
\hline$\%$ Plain & 98.7 & & & & & & & & & \\
\hline \multicolumn{11}{|l|}{ C8 } \\
\hline 0-10 & 59 & & & & & & 1 & & & \\
\hline $10-20$ & 128 & & & & & & & 1 & & \\
\hline $20-30$ & 99 & & & & & & & & & \\
\hline $30-40$ & 148 & & 3 & 1 & & & 6 & & & \\
\hline $40-50$ & 1 & & & & & & & & & \\
\hline $50-60$ & 1 & & & & & & & & & \\
\hline \%Plain & 97.0 & & & & & & & & & \\
\hline \multicolumn{11}{|c|}{ C9 } \\
\hline $0-10$ & 24 & & & & & & 1 & & & \\
\hline $10-20$ & 126 & & & & & & 4 & & & \\
\hline $20-30$ & 208 & & & & & & 12 & 3 & 1 & 1 \\
\hline $30-40$ & 70 & & & & & & 5 & 1 & & \\
\hline \multicolumn{11}{|l|}{$40-50$} \\
\hline \multicolumn{11}{|l|}{$50-60$} \\
\hline \%Plain & 93.2 & & & & & & & & & \\
\hline \multicolumn{11}{|l|}{ C12 } \\
\hline $0-10$ & 30 & & & & & & & & & \\
\hline $10-20$ & 80 & & & & & & 2 & & & \\
\hline $20-30$ & 56 & & 1 & & & 1 & 3 & & & \\
\hline $30-40$ & 97 & & 2 & & & 2 & 4 & & & \\
\hline $40-50$ & 68 & & & & & & 5 & & & \\
\hline \%Plain & 94.0 & & & & & & & & & \\
\hline
\end{tabular}


278 Geoffrey Clark

Table 47 continued

\begin{tabular}{|c|c|c|c|c|c|c|c|c|c|c|}
\hline Square/depth (cm) & Plain sherds & Dentate stamped & Wiped & Side tool & Shell imp. & Dents & $\mathrm{CPI}-/ /$ & CPI-X & Incision & End-tool imp. \\
\hline \multicolumn{11}{|l|}{ C13 } \\
\hline $0-10$ & 60 & & & & & & & & & \\
\hline $10-20$ & 120 & & & 1 & & & & & & \\
\hline $20-30$ & 135 & & & & 1 & 1 & 5 & & & \\
\hline $30-40$ & 87 & & & & & & 1 & & & \\
\hline $40-50$ & 23 & & & & & & & & & \\
\hline \%Plain & 98.6 & & & & & & & & & \\
\hline \multicolumn{11}{|l|}{ D12 } \\
\hline $0-10$ & 22 & & & & & & & & & \\
\hline $10-20$ & 83 & & & & & & & & & \\
\hline $20-30$ & 76 & & & & 1 & & 1 & 1 & & \\
\hline $30-40$ & 79 & & & & & 1 & 4 & 1 & & \\
\hline $40-50$ & 84 & & & & & & & & & \\
\hline \%Plain & 97.4 & & & & & & & & & \\
\hline \multicolumn{11}{|l|}{ D13 } \\
\hline $0-10$ & 56 & & & & & & & & & \\
\hline $10-20$ & 110 & & & & & & & & & \\
\hline $20-30$ & 125 & & & & & 1 & 2 & & & \\
\hline $30-40$ & 79 & & & & & & 1 & & & \\
\hline $40-50$ & 52 & & & & & & & & & \\
\hline $50-60$ & 26 & & & & & & 1 & & 1 & \\
\hline \%Plain & 98.7 & & & & & & & & & \\
\hline \multicolumn{11}{|l|}{ F8 } \\
\hline $0-10$ & 14 & & & & & & & & & \\
\hline $10-20$ & 85 & & & & & & 1 & 1 & & \\
\hline $20-30$ & 80 & & & & & & 3 & 2 & & \\
\hline $30-40$ & 77 & 2 & 1 & & 1 & 2 & 2 & & 1 & \\
\hline $40-50$ & 81 & & & & & & 2 & & & \\
\hline $50-60$ & 54 & & & & & & & & & \\
\hline $60-70$ & 28 & & & & & & 1 & 2 & & \\
\hline$\%$ Plain & 95.2 & & & & & & & & & \\
\hline \multicolumn{11}{|l|}{ G8 } \\
\hline $0-10$ & 20 & & & & & & & & & \\
\hline $10-20$ & 106 & & & & & & 2 & & 1 & \\
\hline $20-30$ & 115 & & & & & & 12 & 2 & 1 & \\
\hline $30-40$ & 104 & & & 1 & & & & & & \\
\hline $40-50$ & 68 & & & 1 & & & 4 & 1 & & \\
\hline \%Plain & 94.7 & & & & & & & & & \\
\hline \multicolumn{11}{|l|}{ H8 } \\
\hline $0-10$ & 8 & & & & & & & & & \\
\hline $10-20$ & 55 & & & & & & 2 & & & \\
\hline $20-30$ & 88 & & & & & & 5 & & & \\
\hline $30-40$ & 75 & & & & & & & 1 & & \\
\hline $40-50$ & 69 & & & & & & & & & \\
\hline \%Plain & 96.6 & & & & & & & & & \\
\hline
\end{tabular}


Table 47 continued

\begin{tabular}{|c|c|c|c|c|c|c|c|c|c|c|}
\hline Square/depth (cm) & Plain sherds & Dentate stamped & Wiped & Side tool & Shell imp. & Dents & (PI-// & CPI-X & Incision & End-tool imp. \\
\hline \multicolumn{11}{|l|}{15} \\
\hline $0-10$ & 26 & & & & & & & & & \\
\hline $10-20$ & 58 & & & & & & & & & \\
\hline $20-30$ & 62 & & & & & & 2 & 2 & & \\
\hline $30-40$ & 183 & & 2 & & & & 3 & 2 & & \\
\hline $40-50$ & 50 & & & & 1 & & & & & \\
\hline \%Plain & 96.8 & & & & & & & & & \\
\hline \multicolumn{11}{|l|}{16} \\
\hline $0-10$ & 28 & & & & & & & & 1 & \\
\hline $10-20$ & 40 & & & & & & 1 & 2 & & \\
\hline $20-30$ & 141 & & & & & & 3 & & & \\
\hline $30-40$ & 77 & & 1 & 1 & & & & 1 & & \\
\hline $40-50$ & 65 & & & & & & & & & \\
\hline \%Plain & 97.4 & & & & & & & & & \\
\hline \multicolumn{11}{|l|}{17} \\
\hline $0-10$ & 5 & & & 1 & & & & & & \\
\hline $10-20$ & 103 & & & 2 & & & 1 & 1 & & \\
\hline $20-30$ & 111 & & & 1 & & & & 2 & & \\
\hline $30-40$ & 39 & & & & & & & & & \\
\hline \multicolumn{11}{|l|}{$40-50$} \\
\hline \%Plain & 98.0 & & & & & & & & & \\
\hline \multicolumn{11}{|l|}{18} \\
\hline $0-10$ & 40 & & & & 1 & & & 2 & 1 & \\
\hline $10-20$ & 8 & & & & & & & & & \\
\hline $20-30$ & 64 & 1 & & & & & & 3 & & \\
\hline $30-40$ & 158 & 3 & 1 & 1 & & & 4 & 2 & & \\
\hline $40-50$ & 60 & & 1 & & & & & 1 & & \\
\hline $50-60$ & 27 & & & 1 & & & & 1 & & \\
\hline $160-70$ & 7 & & & & & & & & & \\
\hline \%Plain & 95.8 & & & & & & & & & \\
\hline \multicolumn{11}{|l|}{19} \\
\hline $0-10$ & 22 & & & & 1 & & & & 2 & \\
\hline $10-20$ & 7 & & & & 12 & & & 1 & 13 & \\
\hline $20-30$ & 68 & & & & 1 & & & 1 & 1 & \\
\hline $130-40$ & 108 & & & & & 1 & 2 & 2 & & \\
\hline $40-50$ & 38 & & & & & & & & & \\
\hline \%Plain & 85.2 & & & & & & & & & \\
\hline \multicolumn{11}{|l|}{110} \\
\hline $0-10$ & 39 & & 1 & & 1 & & & & 1 & \\
\hline $10-20$ & 102 & & & & 10 & & 1 & 6 & 10 & \\
\hline $20-30$ & 124 & & & & 1 & & 2 & 1 & 1 & \\
\hline $30-40$ & 67 & & & 1 & & & & & & \\
\hline $40-50$ & 51 & & 1 & & 1 & & & & & \\
\hline \%Plain & 89.6 & & & & & & & & & \\
\hline
\end{tabular}


280 Geoffrey Clark

Table 47 continued

\begin{tabular}{|c|c|c|c|c|c|c|c|c|c|c|}
\hline Square/depth (cm) & Plain sherds & Dentate stamped & Wiped & Side tool & Shell imp. & Dents & $\mathrm{CPI}-/ /$ & CPI-X & Incision & End-tool imp. \\
\hline \multicolumn{11}{|c|}{ J5 } \\
\hline $0-10$ & 20 & & & 1 & & & & 2 & 1 & \\
\hline $10-20$ & 45 & & 1 & & & & 3 & 5 & 1 & \\
\hline $20-30$ & 62 & & & & & & 5 & 1 & 1 & \\
\hline $30-40$ & 128 & & 1 & & & & 2 & & 2 & \\
\hline $40-50$ & 83 & & & 1 & & & 1 & 2 & & \\
\hline \%Plain & 92.0 & & & & & & & & & \\
\hline \multicolumn{11}{|c|}{ J6 } \\
\hline $0-10$ & 21 & & & & & & & 1 & & \\
\hline $10-20$ & 26 & & & & & & & & & \\
\hline $20-30$ & 60 & & & 1 & & & 1 & & & \\
\hline $30-40$ & 110 & & & & & 1 & 2 & & & \\
\hline $40-50$ & 76 & & & 1 & & & 1 & & & \\
\hline \%Plain & 98.0 & & & & & & & & & \\
\hline \multicolumn{11}{|c|}{$J 7$} \\
\hline $0-10$ & 21 & & & 1 & & & & & & 1 \\
\hline $10-20$ & 51 & 3 & & & & & & & & \\
\hline $20-30$ & 73 & & 4 & & 1 & & 2 & & 1 & \\
\hline $30-40$ & 151 & 1 & & 1 & & & 2 & & & \\
\hline $40-50$ & 47 & & & 1 & & & & & & \\
\hline \%Plain & 95.6 & & & & & & & & & \\
\hline \multicolumn{11}{|l|}{ Ј8 } \\
\hline $0-10$ & 16 & & & & & & & 1 & & \\
\hline $10-20$ & 30 & & & & & & & & & \\
\hline $20-30$ & 44 & 1 & 1 & 1 & & 3 & 2 & & & \\
\hline $30-40$ & 98 & 2 & & 3 & 2 & 1 & & & & \\
\hline $40-50$ & 46 & 1 & 1 & 2 & & & & & & \\
\hline \%Plain & 93.2 & & & & & & & & & \\
\hline \multicolumn{11}{|l|}{ J9 } \\
\hline $0-10$ & 40 & & & & & & & & & 1 \\
\hline $10-20$ & 78 & & & & & & & & & \\
\hline $20-30$ & 104 & 2 & & & & & 2 & 3 & 1 & \\
\hline $30-40$ & 102 & & & & & & 2 & 3 & & \\
\hline $40-50$ & 20 & & 1 & & & & & & & \\
\hline \%Plain & 95.3 & & & & & & & & & \\
\hline \multicolumn{11}{|l|}{ J10 } \\
\hline $0-10$ & 4 & & & & & & & & & \\
\hline $10-20$ & 70 & & & & & & 2 & 1 & & \\
\hline $20-30$ & 77 & & 2 & 1 & & 1 & 3 & 1 & & \\
\hline $30-40$ & 107 & 1 & & 1 & & 1 & 7 & 6 & & \\
\hline $40-50$ & 38 & & & & & & & 1 & & \\
\hline \%Plain & 90.5 & & & & & & & & & \\
\hline
\end{tabular}


Table 47 continued

\begin{tabular}{|c|c|c|c|c|c|c|c|c|c|c|}
\hline Square/depth $(\mathrm{cm})$ & Plain sherds & Dentate stamped & Wiped & Side tool & Shell imp. & Dents & $\mathrm{CPI}-/ /$ & CPI-X & Incision & End-tool imp. \\
\hline \multicolumn{11}{|l|}{$\overline{\mathrm{K} 5}$} \\
\hline $0-10$ & 26 & & & & & & & & & \\
\hline $10-20$ & 65 & & & & & & 1 & 2 & 1 & \\
\hline $20-30$ & 48 & & & & & & 3 & 3 & & \\
\hline $30-40$ & 120 & 1 & & & & 2 & 2 & 4 & & \\
\hline $40-50$ & 63 & & & 2 & & & & & & \\
\hline \%Plain & 94.4 & & & & & & & & & \\
\hline \multicolumn{11}{|l|}{ K6 } \\
\hline $0-10$ & 34 & & & & & & & & & \\
\hline $10-20$ & 56 & & & & & & 1 & & & \\
\hline $20-30$ & 133 & 1 & & 1 & & 1 & 3 & 1 & & \\
\hline $30-40$ & 111 & & & 1 & & 1 & 4 & 2 & & \\
\hline $40-50$ & 80 & 2 & & 2 & 1 & & 4 & & & \\
\hline$\%$ Plain & 94.9 & & & & & & & & & \\
\hline \multicolumn{11}{|l|}{ K7 } \\
\hline $0-10$ & 25 & & & & & & & & & \\
\hline $10-20$ & 31 & & & & & & & & & \\
\hline $20-30$ & 39 & & & & & & & & & \\
\hline $30-40$ & 150 & & & 3 & & & & 2 & & \\
\hline $40-50$ & 52 & & 1 & & & & 1 & 1 & & \\
\hline \%Plain & 98.0 & & & & & & & & & \\
\hline \multicolumn{11}{|l|}{ K8 } \\
\hline $0-10$ & 18 & & & 1 & & & & & & \\
\hline $10-20$ & 47 & & & & & & & & & \\
\hline $20-30$ & 44 & 1 & & & & & 1 & 1 & & \\
\hline $30-40$ & 55 & 4 & 1 & 1 & & & 2 & 3 & 2 & \\
\hline $40-50$ & 56 & & & & & & & & & \\
\hline \%Plain & 90.5 & & & & & & & & & \\
\hline \multicolumn{11}{|l|}{ К9 } \\
\hline $0-10$ & 39 & & & & & & & & & \\
\hline $10-20$ & 106 & & & 1 & & & 2 & 1 & & \\
\hline $20-30$ & 70 & & & & 1 & & & 1 & 2 & \\
\hline $30-40$ & 79 & & & 2 & & & & 4 & & \\
\hline $40-50$ & 59 & & & & & & & 1 & & \\
\hline \%Plain & 95.8 & & & & & & & & & \\
\hline \multicolumn{11}{|l|}{ K10 } \\
\hline $0-10$ & 27 & & & & & & & & & \\
\hline $10-20$ & 92 & & & & & & 1 & & & \\
\hline 20-30 & 87 & & & & & & 1 & 1 & & \\
\hline $30-40$ & 117 & & & 1 & 1 & & 4 & 3 & 1 & \\
\hline $40-50$ & 69 & & 1 & 1 & & & & & & \\
\hline \%Plain & 96.2 & & & & & & & & & \\
\hline \multicolumn{11}{|l|}{ L5 } \\
\hline $0-10$ & 40 & 1 & & 1 & & & 1 & 1 & & \\
\hline $10-20$ & 104 & 1 & & & & & 1 & 3 & & \\
\hline $20-30$ & 147 & 3 & 3 & & 1 & & 6 & 2 & & \\
\hline $30-40$ & 183 & 1 & 4 & 2 & & 1 & 3 & 4 & & \\
\hline \%Plain & 91.1 & & & & & & & & & \\
\hline
\end{tabular}


282 Geoffrey Clark

Table 47 continued

\begin{tabular}{|c|c|c|c|c|c|c|c|c|c|c|}
\hline Square/depth (cm) & Plain sherds & Dentate stamped & Wiped & Side tool & Shell imp. & Dents & CPI-// & CPI-X & Incision & End-tool imp. \\
\hline \multicolumn{11}{|l|}{ L6 } \\
\hline $0-10$ & 12 & & & & & & & & & \\
\hline $10-20$ & 44 & & & & & & & & & \\
\hline $20-30$ & 107 & & & & & & 5 & 7 & & \\
\hline $30-40$ & 102 & 1 & & & 2 & & & & & 1 \\
\hline $40-50$ & 28 & 2 & & 2 & & & 1 & & & \\
\hline \%Plain & 92.2 & & & & & & & & & \\
\hline \multicolumn{11}{|l|}{ L7 } \\
\hline $0-10$ & 23 & & & & & & & & & \\
\hline $10-20$ & 47 & & & & & & & & & \\
\hline $20-30$ & 29 & & & 1 & & & & 1 & & \\
\hline $30-40$ & 97 & 3 & 1 & 1 & & 1 & 2 & 4 & & \\
\hline $40-50$ & 52 & & & & & & & & & \\
\hline \%Plain & 94.4 & & & & & & & & & \\
\hline \multicolumn{11}{|l|}{ L8 } \\
\hline $0-10$ & 5 & & & & & & & 1 & & \\
\hline $10-20$ & 20 & & & & & & & & & \\
\hline $20-30$ & 63 & & & & & & & & & \\
\hline $30-40$ & 80 & 3 & & & & & 3 & & & \\
\hline $40-50$ & 51 & 1 & 1 & 1 & 3 & & & & & \\
\hline $50-60$ & 9 & & & & & & & 1 & & \\
\hline \%Plain & 93.9 & & & & & & & & & \\
\hline \multicolumn{11}{|l|}{ L9 } \\
\hline $0-10$ & 18 & & & & & & & & 1 & 1 \\
\hline $10-20$ & 99 & 1 & & 1 & & & 1 & 1 & & \\
\hline $20-30$ & 151 & & 1 & & & & 5 & & 1 & \\
\hline $30-40$ & 11 & 5 & & 1 & & 1 & 1 & & & \\
\hline $40-50$ & 22 & & & & & 1 & & & & \\
\hline \%Plain & 93.0 & & & & & & & & & \\
\hline \multicolumn{11}{|l|}{ L10 } \\
\hline $0-10$ & 101 & & & & & & & 2 & 1 & \\
\hline $10-20$ & 67 & & & & & & 1 & 1 & & \\
\hline $20-30$ & 90 & & 2 & & & & 2 & 2 & & \\
\hline $30-40$ & 73 & 1 & & 1 & & & 3 & 1 & & \\
\hline 40-50 & 27 & & & & & & & & & \\
\hline \%Plain & 95.0 & & & & & & & & & \\
\hline \multicolumn{11}{|l|}{ M5 } \\
\hline $0-10$ & 42 & & & & & & 2 & & & \\
\hline $10-20$ & 97 & 2 & 2 & 2 & & & 1 & 1 & & \\
\hline 20-30 & 159 & 2 & 2 & 1 & & & 8 & 5 & & \\
\hline $30-40$ & 138 & & & & 1 & & & & & \\
\hline 40-50 & 62 & 1 & & & & & 1 & & & \\
\hline \%Plain & 93.2 & & & & & & & & & \\
\hline
\end{tabular}


Table 47 continued

\begin{tabular}{|c|c|c|c|c|c|c|c|c|c|c|}
\hline Square/depth (cm) & Plain sherds & Dentate stamped & Wiped & Side tool & Shell imp. & Dents & CPI-// & CPI-X & Incision & End-tool imp. \\
\hline \multicolumn{11}{|l|}{ M6 } \\
\hline $0-10$ & 27 & & & & & & & & & \\
\hline $10-20$ & 112 & & & & & & 2 & 5 & & \\
\hline $20-30$ & 164 & 2 & & & & & 9 & 7 & & \\
\hline $30-40$ & 141 & 2 & 1 & 1 & 1 & & 1 & 2 & & \\
\hline $40-50$ & 40 & 2 & & & & & 1 & & & \\
\hline \%Plain & 91.7 & & & & & & & & & \\
\hline \multicolumn{11}{|l|}{ M7 } \\
\hline $0-10$ & 28 & & & & & & 1 & 2 & & \\
\hline $10-20$ & 95 & & & & & & & & & \\
\hline $20-30$ & 112 & & & & & & 1 & 3 & & \\
\hline $30-40$ & 54 & & 1 & 1 & & & 3 & 2 & & \\
\hline $40-50$ & 33 & & & 1 & & & & & & \\
\hline \%Plain & 94.4 & & & & & & & & & \\
\hline \multicolumn{11}{|l|}{ M8 } \\
\hline $0-10$ & 16 & & & & & & & 2 & & \\
\hline $10-20$ & 37 & & & & & & & 1 & & \\
\hline $20-30$ & 73 & 1 & & 1 & & & 3 & 2 & 1 & \\
\hline $30-40$ & 125 & 1 & & 1 & 1 & & 1 & & & \\
\hline $40-50$ & 42 & & & & & & & 1 & & \\
\hline \multicolumn{11}{|l|}{$50-60$} \\
\hline \%Plain & 94.2 & & & & & & & & & \\
\hline \multicolumn{11}{|l|}{ M9 } \\
\hline $0-10$ & 20 & & & & & & & & & \\
\hline $10-20$ & 124 & & 1 & 1 & 1 & 1 & 2 & 1 & & \\
\hline $20-30$ & 127 & 2 & 1 & & & & 4 & & & \\
\hline $30-40$ & 141 & 2 & & & 1 & & 1 & & & \\
\hline $40-50$ & 41 & & & & & & & & & \\
\hline \%Plain & 94.9 & & & & & & & & & \\
\hline \multicolumn{11}{|l|}{ M10 } \\
\hline $0-10$ & 74 & & & & & & & 1 & & \\
\hline $10-20$ & 122 & & & 1 & 1 & & & 1 & & \\
\hline $20-30$ & 112 & 3 & & & & & 1 & 1 & & \\
\hline $30-40$ & 239 & 1 & 2 & 1 & & & 4 & 2 & & \\
\hline $40-50$ & 24 & & & & & & & & & \\
\hline \%Plain & 96.1 & & & & & & & & & \\
\hline \multicolumn{11}{|l|}{ N8 } \\
\hline $0-10$ & 11 & & & & & & & & & \\
\hline $10-20$ & 81 & & & 1 & & & & 1 & & \\
\hline $20-30$ & 96 & 1 & 1 & & & & 1 & 5 & & \\
\hline $30-40$ & 97 & 2 & & 3 & 1 & & 2 & 1 & & \\
\hline 40-50 & 56 & 1 & & 1 & & & 2 & & & \\
\hline $50-60$ & 1 & & & & & & & & & \\
\hline \%Plain & 91.8 & & & & & & & & & \\
\hline
\end{tabular}


284 Geoffrey Clark

Table 47 continued

\begin{tabular}{|c|c|c|c|c|c|c|c|c|c|c|}
\hline Square/depth (cm) & Plain sherds & Dentate stamped & Wiped & Side tool & Shell imp. & Dents & CPI-// & CPI-X & Incision & End-tool imp. \\
\hline \multicolumn{11}{|l|}{ N9 } \\
\hline $0-10$ & 111 & 1 & & 1 & & & 3 & 1 & & \\
\hline $10-20$ & 128 & 4 & 1 & 1 & 1 & & 5 & 3 & & \\
\hline $20-30$ & 92 & 1 & & & 1 & & 2 & 2 & & \\
\hline $30-40$ & 133 & 3 & & & & & 3 & 1 & & \\
\hline $40-50$ & 23 & & & 2 & & & & & & \\
\hline \%Plain & 92.2 & & & & & & & & & \\
\hline \multicolumn{11}{|l|}{ N10 } \\
\hline $0-10$ & 68 & 1 & 1 & 1 & & & & & & \\
\hline $10-20$ & 157 & & & & & & 2 & 1 & & \\
\hline $20-30$ & 100 & 2 & 1 & 2 & 1 & & 6 & 1 & & 3 \\
\hline $30-40$ & 151 & & & 2 & & & 1 & & & \\
\hline $40-50$ & 10 & & & & & & & 1 & & \\
\hline \%Plain & 95.3 & & & & & & & & & \\
\hline \multicolumn{11}{|l|}{08} \\
\hline $0-10$ & 49 & & & & & & 1 & 1 & & \\
\hline $10-20$ & 121 & & & & & & 2 & 2 & & \\
\hline $20-30$ & 229 & 1 & 1 & 1 & 2 & 1 & 5 & 7 & & \\
\hline $30-40$ & 164 & 1 & 1 & 2 & 4 & & 4 & 6 & & 1 \\
\hline $40-50$ & 31 & & & 1 & & & 1 & 1 & & \\
\hline $50-60$ & 5 & & & & & & & & & \\
\hline \%Plain & 92.1 & & & & & & & & & \\
\hline \multicolumn{11}{|l|}{09} \\
\hline $0-10$ & 46 & & & & & & 2 & & & \\
\hline \multicolumn{11}{|l|}{$10-20$} \\
\hline $20-30$ & 109 & & 1 & & & 1 & 4 & 2 & & \\
\hline $30-40$ & 65 & & & 1 & 1 & & 2 & 1 & & 1 \\
\hline $40-50$ & 6 & & & & & & & & & \\
\hline \%Plain & 92.5 & & & & & & & & & \\
\hline \multicolumn{11}{|l|}{010} \\
\hline $0-10$ & 148 & & 1 & & & & 4 & 1 & & \\
\hline $10-20$ & 211 & & 2 & 1 & & 2 & 13 & 2 & & 1 \\
\hline $20-30$ & 112 & & 1 & & & & 1 & 1 & & 1 \\
\hline $30-40$ & 58 & & & & & & & 1 & & \\
\hline $40-50$ & 3 & & & & & & & & & \\
\hline \%Plain & 93.4 & & & & & & & & & \\
\hline \multicolumn{11}{|l|}{ P8 } \\
\hline $0-10$ & 24 & & & & & & & & & \\
\hline $10-20$ & 55 & & & & & & & 2 & & \\
\hline $20-30$ & 142 & & 1 & & & & 4 & 2 & & \\
\hline $30-40$ & 173 & 2 & 1 & & 1 & & 5 & 2 & & \\
\hline $40-50$ & 28 & & & & & & & & & \\
\hline \%Plain & 94.8 & & & & & & & & & \\
\hline
\end{tabular}


Table 47 continued

\begin{tabular}{|c|c|c|c|c|c|c|c|c|c|c|}
\hline Square/depth (cm) & Plain sherds & Dentate stamped & Wiped & Side tool & Shell imp. & Dents & $\mathrm{CPI}-/ /$ & CPI-X & Incision & End-tool imp. \\
\hline \multicolumn{11}{|l|}{ P9 } \\
\hline $0-10$ & 112 & & & & & & & & & \\
\hline $10-20$ & 206 & & 2 & & & & 5 & 5 & & \\
\hline $20-30$ & 143 & 1 & 2 & 1 & 1 & 2 & 8 & & & 1 \\
\hline $30-40$ & 20 & & 1 & & & & & & & \\
\hline $40-50$ & 8 & & & & & & & & & \\
\hline \%Plain & 93.7 & & & & & & & & & \\
\hline \multicolumn{11}{|l|}{ P10 } \\
\hline $0-10$ & 82 & & & & & & 2 & & & \\
\hline $10-20$ & 110 & & & & & & 6 & & & \\
\hline $20-30$ & 102 & & & & & & 2 & & & \\
\hline $30-40$ & 45 & & & & 1 & 1 & & 2 & & \\
\hline $40-50$ & 5 & & & 1 & & & & & & \\
\hline \multicolumn{11}{|l|}{$50-60$} \\
\hline \%Plain & 95.3 & & & & & & & & & \\
\hline \multicolumn{11}{|l|}{ Q10 } \\
\hline $0-10$ & 102 & & & & & & & & & \\
\hline $10-20$ & 90 & & & & & & 1 & 4 & & \\
\hline $20-30$ & 90 & & 1 & & & & & & & \\
\hline $30-40$ & 40 & & & & & & & & & \\
\hline \%Plain & 97.8 & & & & & & & & & \\
\hline \multicolumn{11}{|l|}{ Q13 } \\
\hline $0-10$ & 10 & & & & & & & & 2 & \\
\hline $10-20$ & 7 & & & & & & & & & \\
\hline \multicolumn{11}{|l|}{$20-30$} \\
\hline $30-40$ & 96 & & & & & & & 2 & & \\
\hline \%Plain & 95.6 & & & & & & & & & \\
\hline \multicolumn{11}{|l|}{$\mathrm{T} / \mathrm{U}-1$} \\
\hline $0-10$ & 41 & & & & & & 5 & 1 & & \\
\hline $10-20$ & 57 & & & & & & 3 & 4 & 1 & 1 \\
\hline $20-30$ & 464 & & 1 & 1 & & 1 & 21 & 14 & & \\
\hline $30-40$ & 366 & & & & & 1 & 2 & 2 & & \\
\hline $40-50$ & 74 & & & & & & & & & \\
\hline $50-60$ & 84 & & & & & & & & & \\
\hline \%Plain & 94.5 & & & & & & & & & \\
\hline
\end{tabular}


by the potter using a fibrous material, such as the inner husk of the coconut, on the outer rim during the finishing stages of vessel manufacture. Wiping was identified on 22 rims, of which $76 \%$ belong to Form $1 \mathrm{~A}$ vessels with straight profiles and flat/flat-rounded lips. No wiped bowls were identified, although a plain collar rim has deep striations below the collar. Associated with exterior-rim wiping is one case of interior-lip notching with a side tool. The rim of this vessel is unusual for the depth of the striae and the irregularity of its rim profile and rim-body join.

The use of a side tool to make notches, ovals and cuts is evident on $0.4 \%$ of sherds. Almost $63 \%$ of such sherds were found below $30 \mathrm{~cm}$ depth, and the sherds were in most excavation areas. Two types of side tool were employed. The first had a thin sharp edge that was used to make cuts or apex-down triangular incisions. An impressed diamond shape resulted from moving the edge of the side tool from side-to-side on the lip edge or rim collar. The second tool was oval in cross-section and was used to make small oval impressions 3-8 mm wide on the lip.

Of the 91 sherds with side-tool impression, all but four are rims. The majority of these $(\mathrm{n}=64)$ belong to Form 1B vessels. In all cases, the side tool was applied along the thickest point of the rim and spaced from $0.6 \mathrm{~cm}$ to $1.2 \mathrm{~cm}$. Collar rims were restricted to the main excavation area and like dentate-stamped sherds were not found in the smaller eastern and western excavations. Side-tool marking on Form $1 \mathrm{~A}$ vessels consists of incised lines across the lip and on the interior or exterior lip edge. Form $2 \mathrm{~A}$ and $2 \mathrm{C}$ vessels also have notching and incision on the lip, including one with a crenate-incised lip. Bivalve-edge impressing was found on $0.3 \%$

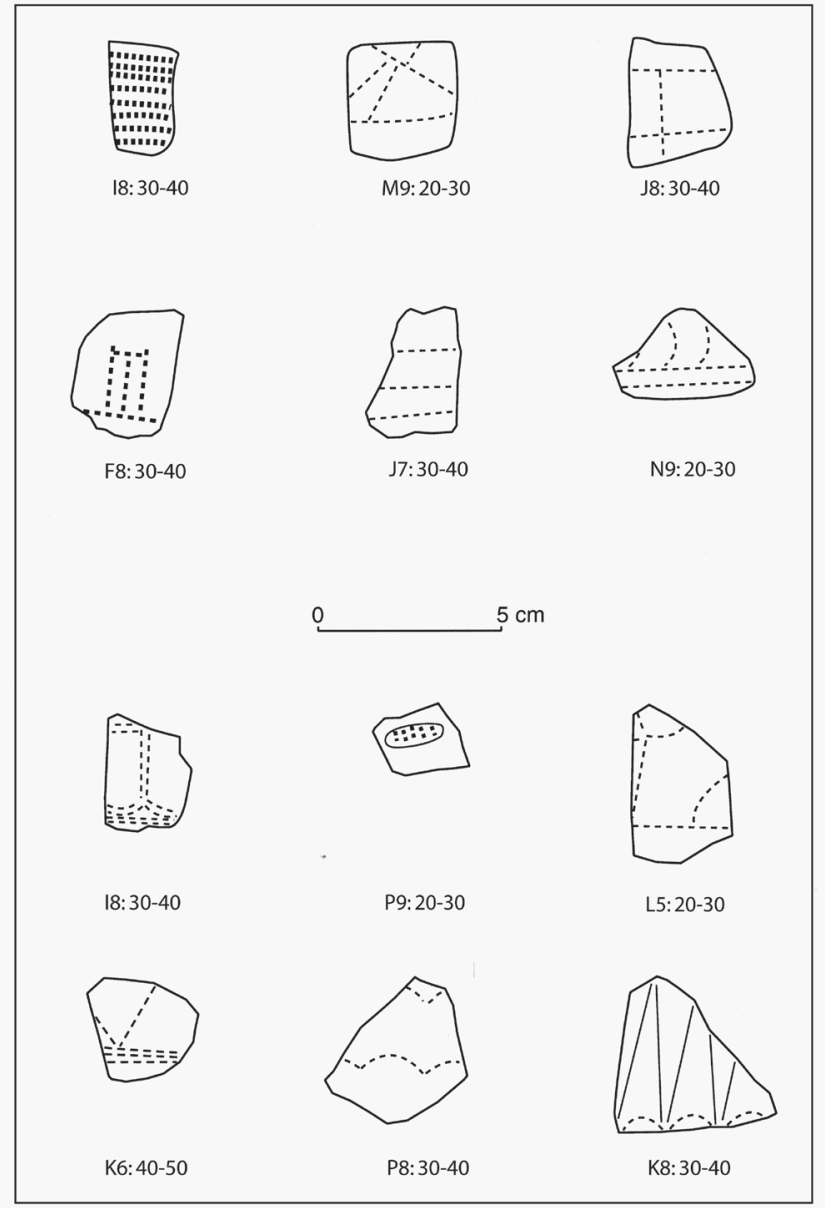

Figure 120. Ugaga Island dentate-stamped sherds (square and depth).

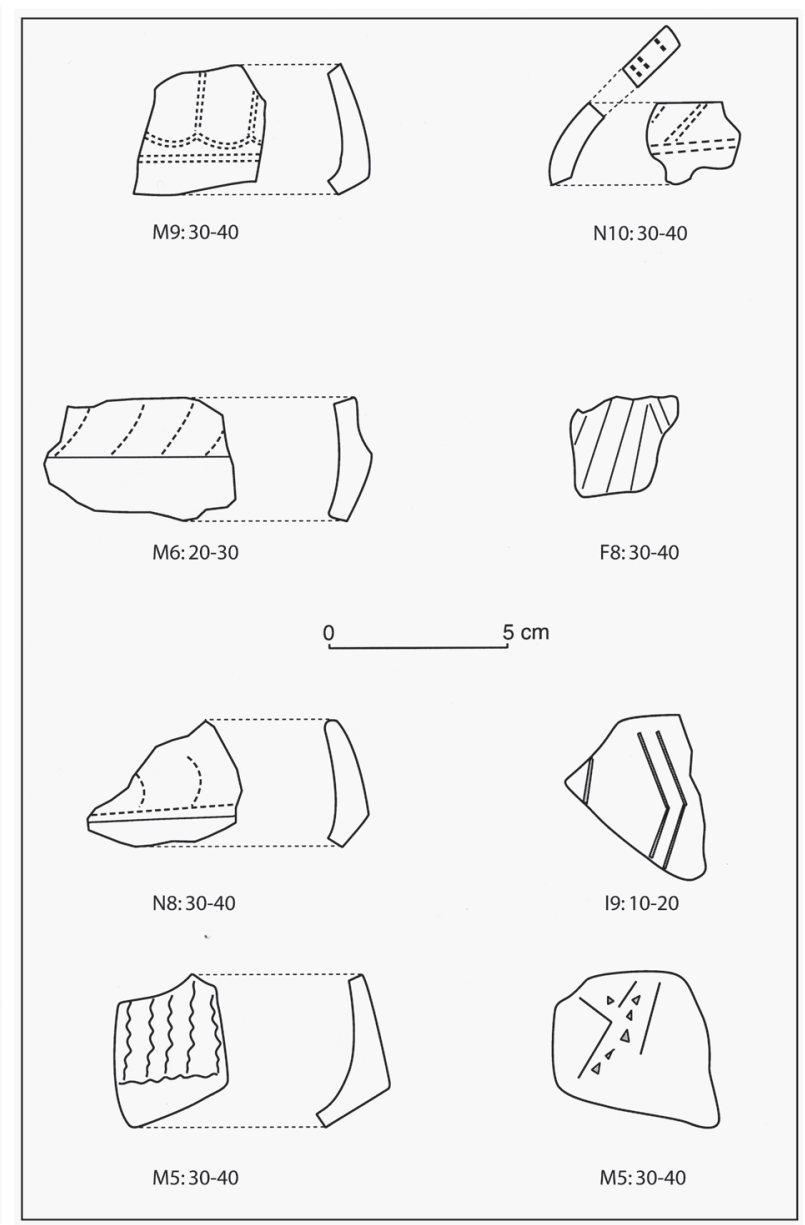

Figure 121. Ugaga Island dentate-stamped, carinated and incised sherds (square and depth). 
of the assemblage. The length of the impression was usually less than $0.6 \mathrm{~mm}$, although a length of $2.2 \mathrm{~cm}$ was recorded from a carinated sherd. Shell impressions were found on 24 Form 1B rims, where they are aligned vertically or diagonally along the widest point of the collar.

Other vessel forms with bivalve-edge impressions are a Form 1A jar and a Form 2C bowl with vertical shell crescents on the rim and small shell impressions on the lip. Most shell-impressed sherds $(\mathrm{n}=28)$ were located in the $10-20 \mathrm{~cm}$ spit of squares I9-I10 and are derived from one vessel. Very few sherds with dents were found $(n=35)$ and the category is difficult to reliably separate from sherds with 'heavy ribs', especially when the sherds are small. Dented sherds were found in all of the excavated areas, mostly at $10-30 \mathrm{~cm}$ depth. In general, dents have straight parallel edges that gradually terminate at one end. The best explanation for this shape is that a flat-rounded or round-edged paddle was used against the vessel surface. Dents were identified on a Form 1A and a Form 2B vessel. Parallel-relief sherds have the highest frequency of any type of surface modification. However, their proportion in the Ugaga assemblage is still small, at $1.9 \%$ of the total assemblage. Sherd numbers were greatest in squares $\mathrm{C} 9$ and O10, and the sherds were found in all excavation areas, with $70 \%$ of them between $20 \mathrm{~cm}$ and $40 \mathrm{~cm}$.

Nine Form 1A vessels have parallel relief on their rim or body. On four of these, the ribs were placed horizontally and are $1.8-2.3 \mathrm{~cm}$ in length. Diagonal parallel impressions of different lengths were found on several vessels. Heavy ribs more than $2 \mathrm{~cm}$ long occur on a Form 1A vessel. Diagonal and horizontal parallel impressions of different sizes were also found on Form $2 \mathrm{~A}$ and $2 \mathrm{~B}$ bowls. Sherds with cross-hatch relief make up $1.2 \%$ of the Ugaga assemblage. They were found in most squares and $78 \%$ were recovered from $20 \mathrm{~cm}$ to $40 \mathrm{~cm}$ depth. The majority of cross-hatch sherds are from the body and only one rim provides any indication of vessel form. Impressions from large shoulder sherds and body sherds indicate that diamond-shaped impressions are vertically orientated. Three size classes (height 3-5 mm, 5-8 mm, 8-12 mm) of square and diamond impressions were recorded from the Ugaga assemblage.

The interior surface of 12 body sherds with cross-hatching (5-8 $\mathrm{mm}$ size class) is rough and pitted. The texture is unlike that produced using a coral anvil (Birks 1973:Plate 36A and 44A). Similar impressions were identified on Level 2 vessels from Sigatoka, where unsmoothed stone anvils were thought to have been used (Birks 1973:42). Impressions from the Ugaga basalt breccia in modelling compound gave a comparable surface texture to that found on the sherds, supporting Birks' identification.

Of the 55 sherds $(0.3 \%)$ with symmetric or asymmetric incision, 28 belong to one vessel. The remainder have single or multiple parallel lines (up to five), although no comb-incised sherds were identified. Crosby (1988:125) records that comb-incising was common in lateprehistoric sites on nearby Beqa. The incision depth ranges from light surface markings to 2 $\mathrm{mm}$ deep cuts. Incision is associated with dentate arcs on one sherd and with triangular endtool impressions on another. A single sherd with asymmetric incision in an 'exploded' chevron pattern was recorded from I9: 10-20 cm.

There are 14 sherds marked by end tools and seven of these came from one vessel marked with circular impressions 2-3 mm in diameter. Other end-tool impressions include an example of finger-nail impressing and oval, square and triangular shapes. Oval to circular impressions, possibly made with a finger tip, were found on Form $1 \mathrm{~B}$ vessels. A rim from a Form $1 \mathrm{~A}$ vessel is unusual for its extremely thickened lip that was marked using a straight-sided tool with a chisel-shaped tip.

Form 1 jars: The majority of Ugaga rims for which the orientation and rim-body contour can be determined (63\% of the assemblage) belong to Form 1 vessels. Rim course is straight 
(47\%) or concave $(52 \%)$, with a single convave (highly everted) rim. Rim profile is divided relatively evenly between parallel (31\%), gradually thickening (23\%) and gradually thinning (20\%) forms. Abruptly thickened rims from Form 1B vessels make up the remaining 26\%. Flat/flat-rounded lips account for $72 \%$ and round/pointed lips $28 \%$ of the 462 undamaged lips. For rim sherds with surface modification, the decoration was most often applied to the rim $(79 \%)$ and less frequently to the lip (14\%), body (6\%), or interior rim. Side tool (notching and cuts) is the most common type of surface modification (46\%), followed by shell impressing (18\%) and wiping (13\%). Present at frequencies below $10 \%$ are paddle relief $(8 \%)$, dentate stamping $(7 \%)$, incision $(6 \%)$, end tool $(2 \%)$ and dents $(1 \%)$.

The mean rim eversion of 282 Form 1 rims is $20^{\circ}$, with a large range, of $2-81^{\circ}$. A histogram plot shows that the data is positively skewed between $2^{\circ}$ and $36^{\circ}$, with a single outlier at $81^{\circ}$. Rim-course measurements $(n=474)$ are normally distributed, with a mean of $7 \mathrm{~mm}$ and a range of 3-14 $\mathrm{mm}$. The height of the rim to the corner point varies from 7 $\mathrm{mm}$ to $63 \mathrm{~mm}$, with a mean of $26 \mathrm{~mm}$. Neck-body inclination angles $(\mathrm{n}=107)$ are $58-150^{\circ}$ (mean $121^{\circ}$ ). The average external rim diameter is $22 \mathrm{~cm}$. Clearly, there is considerable size and shape variation within the Form 1 group.

Form 1B jars with abruptly thickened rims and thinning lips: Eighteen vessels have collar rims (a clay band was applied to the exterior rim surface) or abruptly thickened rims (sharp edged and asymmetric to the exterior). All rim courses are concave. Collar rims vary from thin applied and rounded additions, to large and small applied bands that are triangular or sub-triangular in cross-section. The interior of some Ugaga Form 1B vessels has a rim-body join below the collar rim, suggesting separate manufacture of the thickened rim and body. Lips are mostly round-pointed (58\%), with roughly equal numbers of flat/ flat-rounded and flat/sharp edged. All vessels, except one, are decorated with side-tool notching, shell impressing, or round/oval end-tool impressing at the point of maximum rim thickness. Dentate stamping was found on one vessel with a thickened rim. Form 1B vessels are not strongly everted (mean $16^{\circ}$ ), have a mean rim height of $26 \mathrm{~mm}$, and have an average diameter of $21 \mathrm{~cm}$.

Form 1A jars with aperture diameter 10-20 cm: The majority of the 25 vessels with aperture diameters $10-20 \mathrm{~cm}$ have concave rim profiles (68\%). The most common rim profile is gradual thinning (44\%), with the remainder almost evenly divided between parallel and gradual thickening shapes. Lips are flat/flat-rounded (72\%). Five vessels are decorated on the lip, rim or body. Lip decoration is varied, with side-tool cuts, shell impression, dentate stamping and end tool applied to the lip surface. Two vessels have horizontal parallel relief on the rim and body. Lips are flat/flat-rounded $(72 \%)$ or round/pointed. The mean rim eversion is $25^{\circ}$ and the maximum eversion angle for any vessel in the Ugaga assemblage, of $81^{\circ}$, was found in this group. The average rim height is $24 \mathrm{~mm}$.

Form 1A jars with aperture diameter 21-29 cm: The largest group of Form 1A vessels has aperture diameters of $21-29 \mathrm{~cm}(\mathrm{n}=37)$. In contrast to previous vessel groups, the majority of rim courses are straight (70\%), with convex (24\%) and convave $(6 \%)$ courses in the minority. The distribution of rim profiles is spread fairly evenly between parallel, and gradually thickening or thinning forms. Most lips are flat/flat-rounded (72\%). Sixteen vessels have surface modification, divided between those with modification on the rim or body $(\mathrm{n}=8)$ and vessels where the lip and/or rim is marked. The former group has paddle relief (cross hatch and parallel rib), and the latter group wiping, dentate stamping and side- 
tool notching. Two sorts of side-tool notching were recorded. The first was applied across or diagonally to the lip surface, while the second involves notches on the interior lip-rim surface. Rim eversion is $3-40^{\circ}$ (mean $20^{\circ}$ ). Rim height (mean $37 \mathrm{~mm}$ ) is greater than that of Form 1A vessels, with diameters of $10-20 \mathrm{~cm}$, and of Form 1B vessels.

Form 1A jars with aperture diameters $\geq 30 \mathrm{~cm}$ : Only eight vessels have rim diameters equal to, or greater than, $30 \mathrm{~cm}$. These vessels have straight and concave rim courses and parallel or gradually changing profiles. Most lips are flat or flat-rounded and only one is round/pointed. The body and rim of two vessels are modified by paddle relief and denting. The average rim eversion is $19^{\circ}$. Mean values for rim-course thickness $(7.5 \mathrm{~mm})$ and rim height $(39 \mathrm{~mm})$ are the largest of any sub-group.

Form 2 bowls: There are 72 rims from Form 2 vessels. The number almost certainly underrepresents their real abundance, as larger sherd sizes are required to differentiate Form $2 \mathrm{~A}$ and $2 \mathrm{~B}$ rims from Form $1 \mathrm{~A}$ rims. The majority of rims are Form $2 \mathrm{~A}(70 \%)$, with Forms $2 \mathrm{~B}$ and $2 \mathrm{C}$ contributing the balance. The main rim profile is parallel (44\%), with gradually expanding or contracting profiles present in roughly equal amounts. Lips are flat/flatrounded $(81 \%)$, with a few round/pointed specimens. Surface modification was found on 15 sherds, either on the lip (interior, upper surface, exterior) or body. The limited surfacemodification inventory consists of side-tool cuts $(n=8)$, parallel rib $(n=5)$, shell impressions and dents. The mean eversion angle is $14^{\circ}$ and for inverted rims $21^{\circ}$. The mean diameter is $24 \mathrm{~cm}$, which is similar to that of the total Form 1 sample.

Form 2A everted rim/direct rim-body contour: There are 13 Form $2 \mathrm{~A}$ vessels. These have straight rim courses and gradually changing or parallel rim $(n=5)$ profiles. Most lips are flat/flat-rounded (77\%). Five vessels have surface modification that was placed on the lip (interior and exterior) and body. The surface modification consists of parallel-rib relief and side-tool cuts $(\mathrm{n}=3)$. Eversion angles range from $5^{\circ}$ to $31^{\circ}$, with a mean of $20^{\circ}$, and the average rim diameter is $25 \mathrm{~cm}$.

Form 2B inverted rim/direct rim-body contour: Five of six Form 2B vessels have evenly inverted rim courses and one is straight. Rim profiles are parallel $(n=5)$ or gradually thickened. Surface modification of parallel relief and dents occurs on the bodies of five vessels. Four of these vessels are similar to one another in shape, diameter $(19-20 \mathrm{~cm})$ and rim inversion angle $\left(31-37^{\circ}\right)$.

Form 2C inverted rim/indirect rim-body contour: Three Form 2C bowls with pointed/ rounded lips are decorated on the exterior or interior lip. Decoration consists of shell impressions and side-tool cuts. The diameter range is $18-34 \mathrm{~cm}$.

\section{Form 3 flat-based vessels}

Form $\mathbf{3 A}$ flat-based vessel diameter $<\mathbf{3 0} \mathbf{~ c m}$ : Eight sherds from a dentate-stamped dish with outcurving sides and a flat base were found in Squares J10, K6, K8, L6, L8 and L9 at depths between $30 \mathrm{~cm}$ and $50 \mathrm{~cm}$. The base diameter is calculated at $20 \mathrm{~cm}$ and the estimated diameter of the exterior rim is $22 \mathrm{~cm}$. Anson motifs 314 and 366, both previously found in Reef/Santa Cruz assemblages (Anson 1983), are evident between single and double horizontal rows of dentate stamping. The tools used were thin (approximately $1 \mathrm{~mm}$ wide) and had up to six toothed projections per centimetre. 


\section{Form 4 narrow-orifice vessels}

Form 4B double orifice: A single double-spouted vessel has a central orifice $23 \mathrm{~mm}$ in diameter and a small spout with an estimated opening of $5 \mathrm{~mm}$ above or on the vessel shoulder. This thin-walled vessel is decorated with a horizontal applied band and shell impressing within radiating incised lines. This vessel is similar to the historically known gusui rua (Palmer 1971:Fig. 1; Rossitto 1990).

Form 4C double orifice and stirrup handle: Two of the three vessels with spouts belong to double-spouted and stirrup-handled forms also identified at Navatu. Internal spout diameters are $7-10 \mathrm{~mm}$.

Carinations, handles, ?lugs and ?stands: A mean angle of $132^{\circ}$ was found for 10 carinated sherds (range $108-155^{\circ}$ ). Five are decorated with dentate stamping or shell impressing. Three kinds of handles are tentatively identified. The first is a vertical handle with a height of $30 \mathrm{~mm}$, a flat top and a diameter of $15-30 \mathrm{~mm}$. Side-tool markings are visible on two specimens. It is possible that what have been identified as 'handles' are, in fact, legs or other kinds of vessel projection. However, in one case the sherd thickness below the handle is only $4.5 \mathrm{~mm}$, suggesting a lid function. Two handles with narrow oval cross-sections are likely to derive from the apex of stirrup handles from double-spouted vessels. Two ?handle fragments might be from narrow-orifice Form $4 \mathrm{~A}$ vessels with strap handles similar to those found at Sigatoka Level 1 (Birks 1973:119). Sherds identified as 'lugs' are most frequently 'tongue' or oval shaped in plan view and vary significantly in thickness. Irregularly shaped sherds provisionally classified as ?stands could also be fragments from vessels with thick and uneven rims, or from vessels with currently unrecognised morphology.

\section{Karobo ceramics}

More than $82 \%$ of sherds from the Karobo assemblage have some form of surface modification (Tables 48-49). Just over 70\% of sherds identifiable to Form 1A jars or Form 2 bowls have relief surface modification, indicating that most of the non-platter ceramics were marked with cross hatch or parallel relief. Side tool, end tool and incision are extremely rare in the collection. Sherds with cross-hatch relief comprise the largest group, at $42 \%$ of the decorated sherds (Figure 122). Three size classes of diamond-shaped impression are recorded. The largest of these has a length of $1.2 \mathrm{~cm}$ and width of $0.6 \mathrm{~cm}$, the next size class has a length of $1.0 \mathrm{~cm}$ and width of $0.4-0.5 \mathrm{~cm}$, and the smallest diamond impressions from the assemblage have a length of 0.7 $\mathrm{cm}$ and width of $0.3-0.4 \mathrm{~cm}$. Dimensions of square impressions are ca. $0.8 \mathrm{~cm}, 0.5 \mathrm{~cm}$ and 0.2 $\mathrm{cm}$. Sherds with linear relief (Figure 123) comprise $18.9 \%$ of the Karobo assemblage. Parallel impressions are divided into three forms on the basis of their width and interval spacing. Thin ribs $-0.5-1 \mathrm{~mm}$ wide separated by $0.5-3 \mathrm{~mm}$ gaps - are lightly impressed on a vessel, and on one sherd the impressions are vertically positioned. Medium ribs - with a spacing of $2-4 \mathrm{~mm}$ and a variable width of 1.5-3.7 $\mathrm{mm}$ - are unevenly aligned and inspection under low-power magnification suggests the tool used to groove the paddle had a straight or slightly curved edge. Long and irregular parallel impressions are rare, occurring on fewer than 15 sherds. These impressions have low and irregular borders that are 4-7 mm wide and spaced 4-6 mm apart. Rather than incisions on a paddle's surface, these impressions might have been created using the edge of a paddle or baton against the vessel.

Only 11 sherds, or $1.1 \%$ of the Karobo assemblage, are decorated by incision, or end or side tool. Incision is symmetric, and except in one case consists of single or parallel lines. Although 
Table 48. Karobo ceramics: Sherd type by excavation square and depth.

\begin{tabular}{|c|c|c|c|c|c|c|c|c|}
\hline Excavation/ layer & Plain body & Decorated body & Rim & Neck & Platter rims & Platter body & Total & Weight (g) \\
\hline A2: Layer 1 & 2 & & & & & 12 & 14 & 168 \\
\hline A2: Layer 2 & 9 & 7 & & & & & 16 & 161 \\
\hline A2: Layer 3 & & 6 & & & & 8 & 14 & 175 \\
\hline \multicolumn{9}{|l|}{ A2: Layer 4} \\
\hline A2: Layer 5 & 136 & 415 & 24 & 45 & 62 & 372 & 1054 & 27979 \\
\hline Total & 147 & 428 & 24 & 45 & 62 & 392 & 1098 & 28483 \\
\hline \multicolumn{9}{|l|}{ Aa: Layer 1} \\
\hline \multicolumn{9}{|l|}{ Aa: Layer 2} \\
\hline Aa: Layer 3 & 7 & 1 & & & & 17 & 25 & 277 \\
\hline Aa: Layer 4 & 5 & 4 & & 1 & & 5 & 15 & 72 \\
\hline Aa: Layer 5 & & & & & & 8 & 8 & 315 \\
\hline Aa: Layer 6 & & & & & 1 & 7 & 8 & 255 \\
\hline Aa: Layer 7 & 34 & 75 & 4 & 5 & & 54 & 172 & 3536 \\
\hline Total & 46 & 80 & 4 & 6 & 1 & 91 & 228 & 4455 \\
\hline \multicolumn{9}{|l|}{ Ab: Layer 1} \\
\hline \multicolumn{9}{|l|}{ Ab: Layer 2} \\
\hline Ab: Layer 3 & & 18 & 1 & 1 & & 28 & 48 & 542 \\
\hline Ab: Layer 4 & 1 & & 2 & 1 & & & 4 & 25 \\
\hline Ab: Layer 5 & & & & & & 22 & 22 & 178 \\
\hline Ab: Layer 6 & 5 & 1 & & & & 5 & 11 & 104 \\
\hline Ab: Layer 7 & 10 & 28 & 8 & 8 & 17 & 157 & 228 & 6974 \\
\hline Total & 16 & 47 & 11 & 10 & 17 & 212 & 313 & 7823 \\
\hline Y: Layer 3 & & 19 & & & & & 19 & 102 \\
\hline Y: Layer 4 & 18 & 122 & 1 & 3 & & & 144 & 1135 \\
\hline Total & 18 & 141 & 1 & 3 & & & 163 & 1237 \\
\hline Site Total & 227 & 696 & 40 & 64 & 80 & 695 & 1802 & 41998 \\
\hline
\end{tabular}

Table 49. Karobo ceramics: Sherd decoration by excavation square and depth.

\begin{tabular}{|c|c|c|c|c|c|c|c|c|c|}
\hline Excavation/ layer & CPI-X & CPI-// & CPI-? & Leaf imp. & Mat imp. & ?Leaf/Mat & Incised symm. & End tool & Side tool \\
\hline A2:Layer 1 & & & & 12 & & & & & \\
\hline A2:Layer 2 & 6 & 1 & & & & & & & \\
\hline A2:Layer 3 & & & 3 & 7 & 1 & & & & \\
\hline \multicolumn{10}{|l|}{ A2:Layer 4} \\
\hline A2:Layer 5 & 277 & 103 & 45 & 393 & 7 & 35 & 3 & 2 & 1 \\
\hline \multicolumn{10}{|l|}{ Aa:Layer 1} \\
\hline \multicolumn{10}{|l|}{ Aa:Layer 2} \\
\hline Aa:Layer 3 & 1 & & & 17 & & & & & \\
\hline Aa:Layer 4 & 4 & & & 5 & & & & & \\
\hline Aa:Layer 5 & & & & 8 & & & & & \\
\hline Aa:Layer 6 & & & & 8 & & & & & \\
\hline Aa:Layer 7 & 46 & 21 & 9 & 54 & & & 1 & 2 & \\
\hline \multicolumn{10}{|l|}{ Ab:Layer 1} \\
\hline \multicolumn{10}{|l|}{ Ab:Layer 2} \\
\hline Ab:Layer 3 & 8 & 6 & 4 & 28 & & & & & \\
\hline \multicolumn{10}{|l|}{ Ab:Layer 4} \\
\hline Ab:Layer 5 & & & & 22 & & & & & \\
\hline Ab:Layer 6 & 1 & & & & & & & & \\
\hline Ab:Layer 7 & 26 & 2 & 4 & 168 & 6 & & & & \\
\hline Y:Layer 3 & 6 & 5 & 8 & & & & & & \\
\hline Y:Layer 4 & 52 & 56 & 14 & & & & & & \\
\hline Site Total & 427 & 194 & 87 & 722 & 14 & 35 & 4 & 4 & 1 \\
\hline
\end{tabular}


superficially similar to comb incising, three of the four sherds have a smooth-rounded crosssection unlike the irregular and flat-bottomed channels made with a comb. A single case each of side-tool notching and finger impression was found on sherds of unknown vessel form. A rim sherd with a mat-impressed surface was also found, with the impressions similar to those made using a Pandanus sp. mat (see below). Almost all sherds (99.5\%) from flat-based platters have leaf or mat impressions on their base. Leaf impressions are prominent (93\%), with a smaller number of mat impressions (1.8\%). Palmer (1965a:28) recorded two kinds of leaf impression from the Karobo platters, one from a banana leaf (Musa sp.), while the other was not identified. In a detailed study of the leaf impressions found on similar vessels from Level 2 of Sigatoka, Lambert (1971) identified Hibiscus, Macaranga and Aleurites moluccana impressions, using leaf shape and venation patterns from modern reference material. In that study, Lambert records that the Karobo leaf impressions were identified as Macaranga magna. Leaf impressions at Karobo occur on the base and rim but are often indistinct. The few sherds with clear leaf markings are compatible with Lambert's species attribution. The impressions have parallel, evenly spaced primary veins attached to a midrib, vein termination in a shallow deltoid shape at the laminal edge, and secondary veins that are rectangular and evenly spaced between the major veins. According to Parham (1964), Macaranga magna is a common forest tree in wet zones like the south Viti Levu coast.

Mat impressions on platter sherds are of two sizes and restricted to the exterior of platter bases. The stem width is $2.5-3.5 \mathrm{~mm}$ on the smaller and $4-6 \mathrm{~mm}$ on the larger. Lambert (1971:133) records that in some cases impressions from Pandanus mats can be identified. The

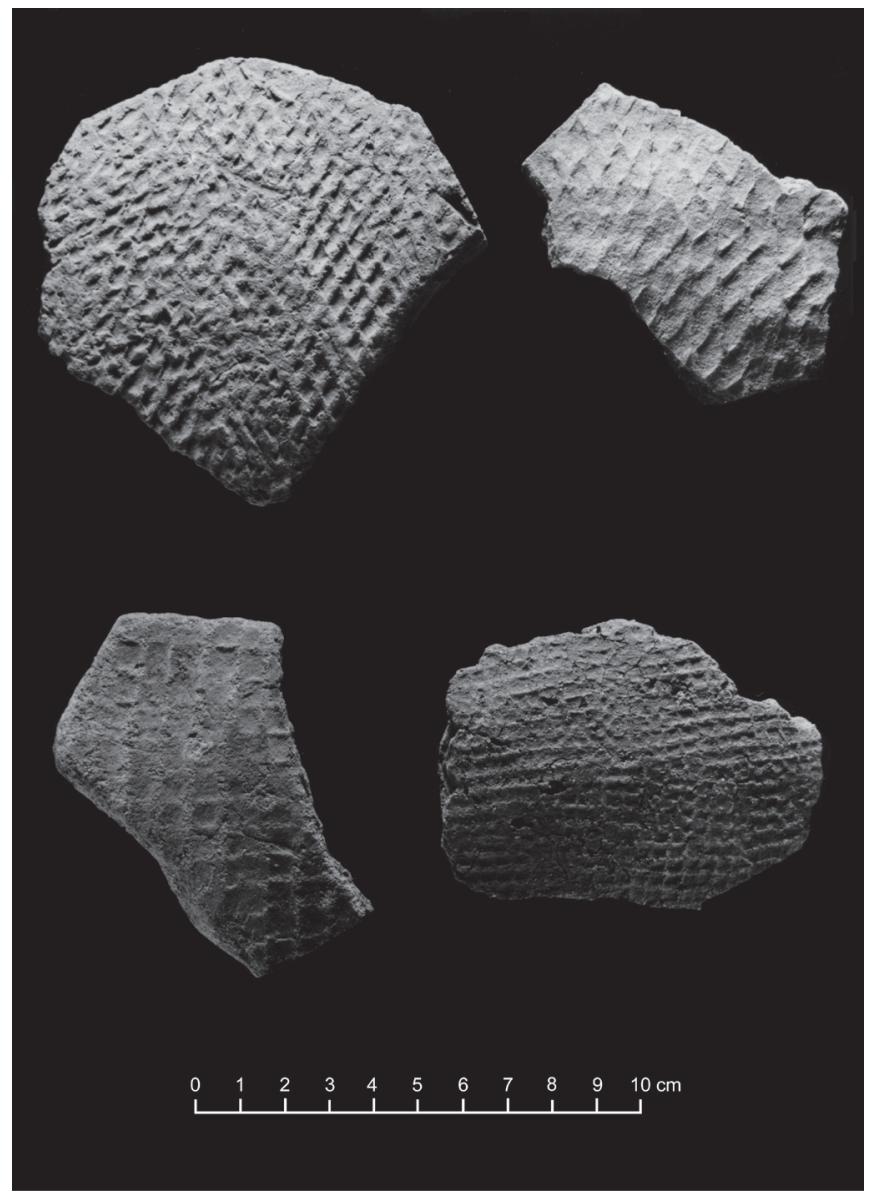

Figure 122. Karobo VL 18/1 sherds decorated with cross-hatch relief.

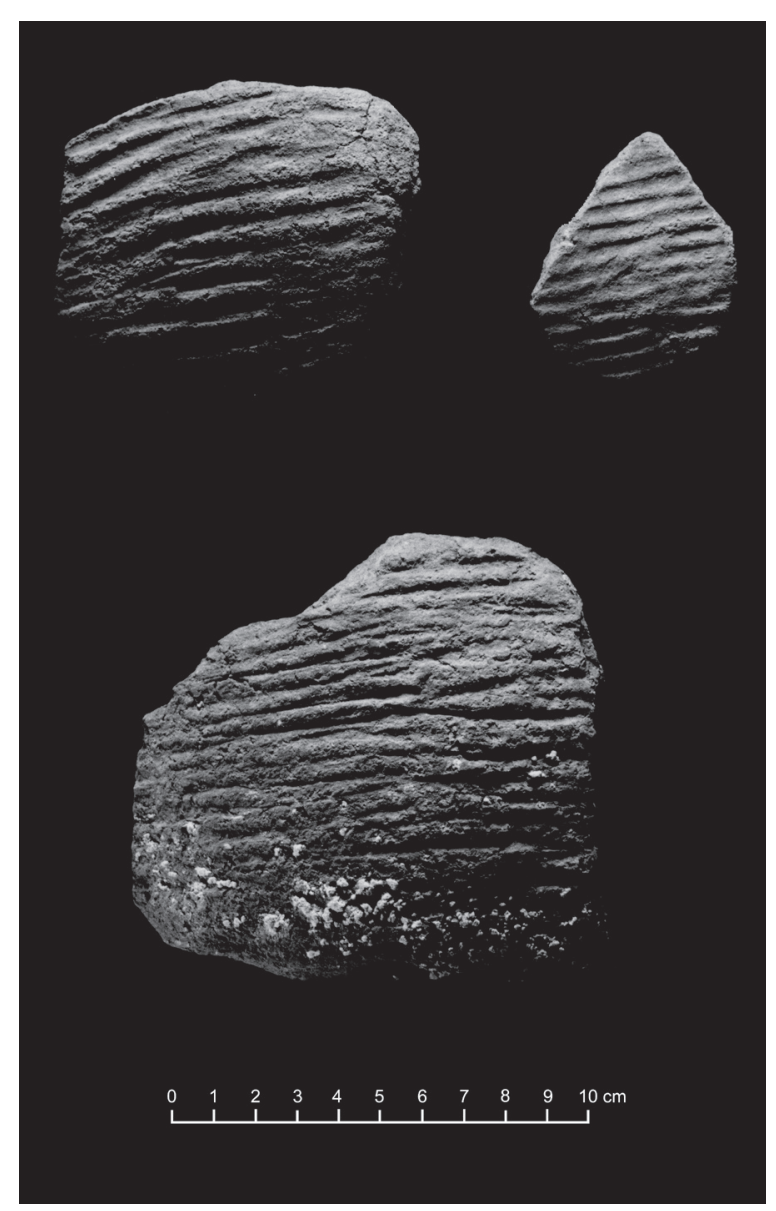

Figure 123. Karobo VL 18/1 sherds decorated with parallelrib relief. 
lower surface of the Pandanus leaf is marked by evenly spaced longitudinal ridges, while the upper surface is smooth. Other sedges used to make mats such as Eleocharus sp. have a different surface structure (Lambert 1971:Plate X). When mat impressions display alternating smooth and fineridged sections it is likely the mat was made from Pandanus sp. Impressions taken from matimpressed sherds displayed alternating smooth and ridged surfaces, indicative of Pandanus mats.

Form 1A jars: Almost all rims from non-platter vessels (93\%) belong to Form 1A vessels. The rim course is straight $(57 \%)$ or slightly concave $(43 \%)$, and the rim profile is parallel $(55 \%)$ or gradual thinning $(37 \%)$ or displays a small amount of proximal thickening $(8 \%)$. There are two lip shapes: flat/flat-rounded forms, oriented normal to the rim axis, account for $88 \%$, and round/pointed $12 \%$. Rim decoration is limited to a single case of circular punctate on the dorsal lip and 18 sherds with cross-hatch and parallel relief on the rim. The mean eversion angle from 45 Form $1 \mathrm{~A}$ rims is $21^{\circ}$, with a range of $14-33^{\circ}$. The rimcourse thickness $(\mathrm{n}=49)$ varies from $4.4 \mathrm{~mm}$ to $13.8 \mathrm{~mm}$, with a mean of $7.8 \mathrm{~mm}$, and the rim height average is $33 \mathrm{~mm}$ (range 12-52 $\mathrm{mm}$ ). The average neck inclination angle of 34 rims is $133^{\circ}$ (range $100-156^{\circ}$ ). The vessel diameter (34 rims) is $18-32 \mathrm{~cm}$, with a mean of $25 \mathrm{~cm}$.

Form 2 bowls: Rims from three Form 2 vessel forms are identified. The first is from a Form $2 \mathrm{~B}$ vessel with a flat lip normal to the rim axis and rounded edges. The vessel has cross-hatch relief and an estimated diameter of $29 \mathrm{~cm}$. Another Form 2B represents a bowl with a flat lip and diameter of $32-35 \mathrm{~cm}$. The final vessel form is an unusual Form $2 \mathrm{C}$ with a slightly inverted rim. Lip shape is flat and the diameter is $29 \mathrm{~cm}$.

Form 3 flat-based vessels: As noted by Birks (1973:44), the rim height of flat-based vessels can vary by $50 \%$ on the same vessel. Lip form varies from gradual to sharply tapering points. An additional source of rim-form variation is found in corner sections that are shallow and rounded in cross-section. At Karobo, the base of these vessels appears to have been formed by rolling out slabs of clay on top of a leaf or mat covering (base thickness $6.7-23.4 \mathrm{~mm}$ ). A slab or cylinder of clay was then shaped into a triangular-sectioned piece that was fixed and smoothed to the basal slab. In contrast to the irregular surface of the exterior base, the interior surface was smoothed. The rim to base height ranges from $2.7 \mathrm{~mm}$ to $6.6 \mathrm{~mm}$, with a mean height of $40 \mathrm{~mm}$. While platter form is difficult to determine, it is likely that in plan view they have straight or slightly curving sides and rounded corners. The diameter of four Karobo platters is estimated from large rim and base sections at approximately $50-53 \mathrm{~cm}$. The estimate is consistent with plan dimensions from three platters from Level 2 at Sigatoka (ca. $52 \mathrm{~cm}, 67 \mathrm{~cm}, 76-78 \mathrm{~cm}$ ). A minimum of three or probably four platters are present in the Karobo deposits.

Form 4 narrow-orifice vessels: A single example of a double-spouted vessel (Form 4A) was found at Karobo. The sherd has a flat handle and orifice diameters of $9 \mathrm{~mm}$ and $12 \mathrm{~mm}$. The sherd does not have a provenance and was probably collected from the surface of the site. It has an internal spout diameter of $15 \mathrm{~mm}$ and a narrow handle diameter of $24 \mathrm{~mm}$. Two spouts from Karobo are also unlocalised, but probably derive from surface collections. One of these might have been from a double-spouted vessel.

Perforated clay object: An unusual find at Karobo was a perforated clay fragment that would have been roughly circular when complete. Maximum dimensions of the piece are $8.5 \mathrm{~cm} \times 8.2 \mathrm{~cm}$ (thickness $4.2 \mathrm{~cm}$ ). The tapered perforation has a diameter of $20 \mathrm{~mm}$ at the top and $15 \mathrm{~mm}$ at the bottom. The function of this object is not known. 


\section{Votua ceramics}

A total of 1400 sherds weighing $5.8 \mathrm{~kg}$ was recovered from the Area 1 shell midden excavation with more than $80 \%$ of the sherds, by weight and number, coming from the surface to 20 $\mathrm{cm}$ depth (Table 50). Plain body sherds make up $82 \%$ of the assemblage, and the mean sherd thickness of a sherd sub-sample is $6.8 \mathrm{~mm}$. The incidence of surface modification is low, with 38 , or $2.7 \%$, of sherds marked, but the majority have traces of a red slip/wash. Seventeen sherds have coarse wipe marks made using coconut husk fibre or similar material. Dentate stamping on eight sherds consists of simple horizontal, diagonal and intersecting straight lines and arcs. The single motif (Figure 124) is a repeated loop, bordered above and below by a single horizontal line of dentate stamping, a motif similar to M46 of Mead et al. (1973). Remaining decorated sherds consist of single or double-impressed lines made using the edge of a shell bivalve, and a single notched and applied band. Several lug/handle sherds are also recorded (Figure 125). There are 157 rim sherds, most (54\%) of which come from vessels with an everted rim and indirect body contour characteristic of 'jar' vessel forms. Vessels with inverted or direct rims and direct body contours ('bowls') make up $26 \%$ of all rims. The Area 1 vessel forms reconstructed from the largest rim sherds are shown in Figure 126, which shows a diverse but not uncommon Lapita assemblage composed of large and small diameter bowls, jars with strongly everted rims, rounded or flat lips and sub-globular bodies, jars with collar rims and shallow carinated dishes.

Table 50. Votua (1996) ceramics: Sherd type by excavation square and depth.

\begin{tabular}{|c|c|c|c|c|c|c|c|c|}
\hline Excavation/ depth $(\mathrm{cm})$ & Plain body & Decorated body & Rims & Necks & Carination & Handle/lug & Number & Weight (g) \\
\hline$A 1: 0-10$ & 315 & 1 & 43 & 8 & 2 & 2 & 371 & 1510 \\
\hline$A 1: 10-20$ & 29 & 5 & 13 & 3 & & & 50 & 230 \\
\hline $\mathrm{A} 1: 20-30$ & 1 & 10 & & & 1 & & 82 & 340 \\
\hline Total & 414 & 7 & 66 & 11 & 3 & 2 & 503 & 2080 \\
\hline$A 2: 0-10$ & 480 & 5 & 52 & 51 & 1 & 2 & 545 & 2290 \\
\hline$A 2: 10-20$ & 241 & 1 & 33 & & 1 & 1 & 277 & 1110 \\
\hline$A 2: 20-30$ & 48 & 1 & 6 & & & & 55 & 290 \\
\hline$A 2: 30-40$ & 20 & & & & & & 20 & 50 \\
\hline Total & 789 & 7 & 91 & 5 & 2 & 3 & 897 & 3740 \\
\hline TP1:0-10 & 122 & & 10 & & & & 132 & 510 \\
\hline TP1:10-20 & 140 & & 6 & 2 & & & 148 & 530 \\
\hline TP1:20-30 & 152 & & 11 & 1 & & & 164 & 740 \\
\hline TP1:30-40 & 190 & & 18 & 2 & & 1 & 211 & 980 \\
\hline TP1:40-50 & 447 & & 57 & 3 & & 4 & 511 & 2610 \\
\hline TP1:50-60 & 69 & & 11 & 1 & 2 & & 83 & 390 \\
\hline TP1:60-70 & 68 & 7 & & & & & 75 & 470 \\
\hline Total & 1188 & & 120 & 9 & 2 & 5 & 1324 & 6230 \\
\hline
\end{tabular}

\section{Malaqereqere ceramics}

The small pottery assemblage from Malaqereqere was recovered from excavation units A1 and A2. The two units were excavated to a depth of $60-70 \mathrm{~cm}$. The total collection consists of 1057 sherds weighing $3.2 \mathrm{~kg}$ (Table 51). Almost the entire ceramic assemblage shows signs of burning on the interior and exterior of sherds. This black discolouration makes the identification of burnishing or slip application difficult, and sherds are small, indicating substantial fragmentation. The distribution of plain body sherds shows a peak at 50-60 cm depth in both test pits. Ceramic 

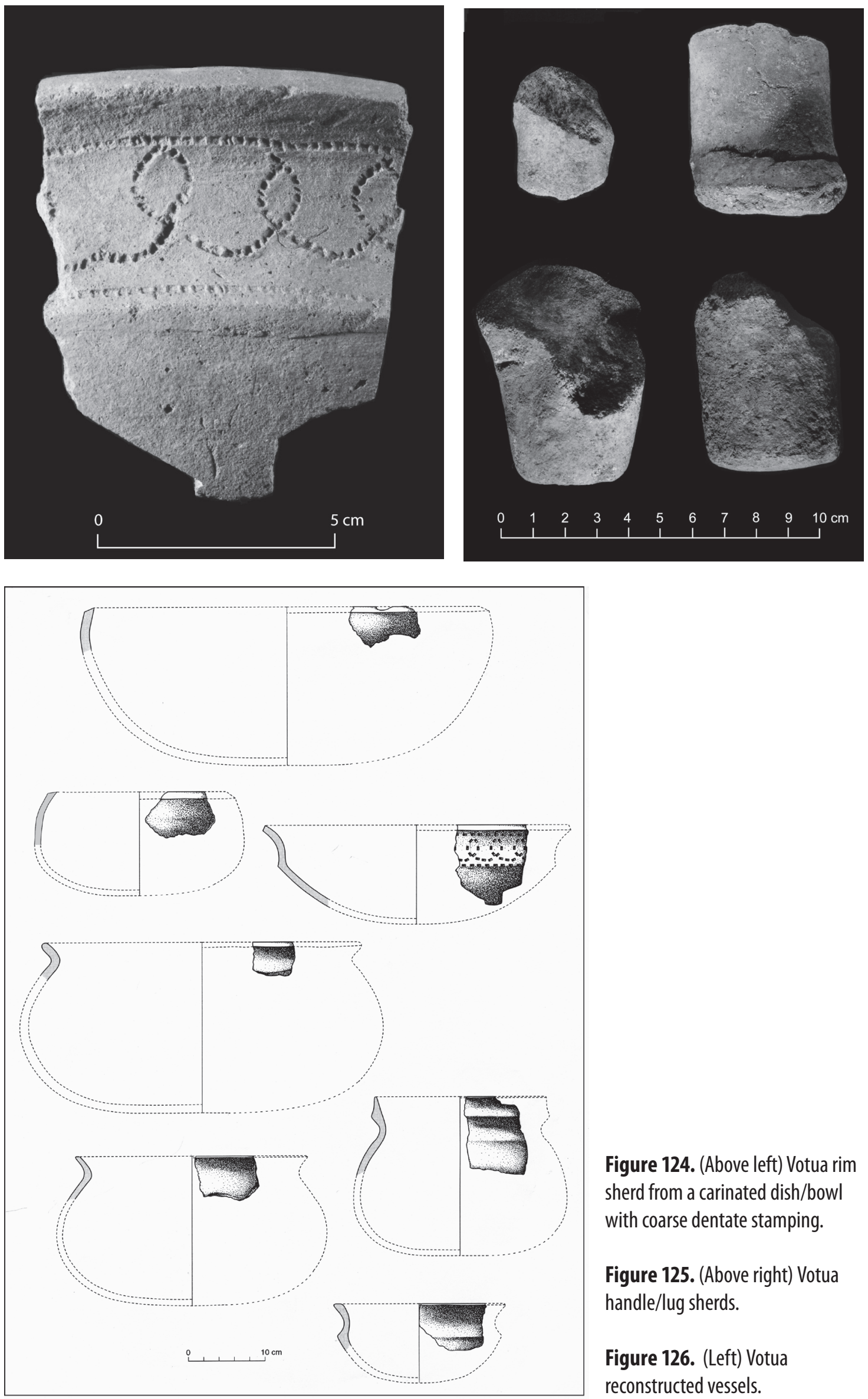

Figure 124. (Above left) Votua rim sherd from a carinated dish/bowl with coarse dentate stamping.

Figure 125. (Above right) Votua handle/lug sherds.

Figure 126. (Left) Votua reconstructed vessels. 
decoration is rare, with examples of incision, end-tool impression and fingernail impression, which are all indicative of late prehistoric ceramics. However, a single dentate-stamped sherd was found at 50-60 cm depth in Square A1. Identifiable rim sherds are from Form 1A jars with parallel or thinning profiles and pointed and flat lips. One Form 1A is from a typical lateprehistoric kuro style cooking pot with a sharply everted rim and an aperture diameter of 28 $\mathrm{cm}$.

Table 51. Malaqereqere ceramics: Sherd type by excavation square and depth.

\begin{tabular}{|c|c|c|c|c|c|c|}
\hline Excavation/depth $(\mathrm{cm})$ & Plain body & Decorated body & $\operatorname{Rim}$ & Neck & Number & Weight (g) \\
\hline A1:0-10 & 49 & & 2 & & 51 & 246.3 \\
\hline A1:10-20 & 36 & & 1 & & 37 & 179.7 \\
\hline A1:20-30 & 26 & & & & 26 & 125.2 \\
\hline A1:30-40 & 17 & & & & 17 & 55.6 \\
\hline A1:40-50 & 73 & 5 & & 1 & 79 & 252.1 \\
\hline A1:50-60 & 228 & 6 & 1 & & 235 & 603 \\
\hline A1:60-70 & 82 & & 1 & & 83 & 221.8 \\
\hline Total & 511 & 11 & 5 & 1 & 528 & 1683.7 \\
\hline A2:0-10 & 34 & & 3 & & 37 & 167.5 \\
\hline$A 2: 10-20$ & 21 & & & & 21 & 104.9 \\
\hline$A 2: 20-30$ & 23 & 1 & & & 24 & 84.8 \\
\hline A2:30-40 & 25 & & & & 25 & 71.4 \\
\hline A2:40-50 & 59 & 1 & & & 60 & 176.1 \\
\hline A2:50-60 & 332 & 1 & 3 & & 336 & 880.3 \\
\hline A2:60-70 & 26 & & & & 26 & 41.8 \\
\hline Total & 520 & 3 & 6 & & 529 & 1526.8 \\
\hline
\end{tabular}

\section{Volivoli II ceramics}

The pottery assemblage from Volivoli II is small, consisting of 1326 sherds, weighing $6.89 \mathrm{~kg}$ (Table 52). Most are plain body sherds (93.5\%). The density of pottery was highest in the top $60 \mathrm{~cm}$ and declined below, with only a few possibly intrusive sherds found below $100 \mathrm{~cm}$ depth. Decoration consists of comb incised and tool incised $(10-30 \mathrm{~cm}$ ), with paddle impression (cross hatch, parallel rib and diamond) from $20-50 \mathrm{~cm}$ depth. Rims are from Form 1A jars (15 sherds from six vessels), Form 2A bowls (three sherds from three vessels) and a Form 2B bowl (one sherd from one vessel). Rims from all vessel forms have a profile that is parallel or thinning, and lip forms that are flat-sharp edged or flat-rounded.

\section{Volivoli III ceramics}

The small pottery assemblage from Volivoli III consists of 371 sherds, weighing $3.33 \mathrm{~kg}$ (Table 53). The vast majority of these $(94.5 \%)$ are plain body sherds. Only two body sherds from Spit 3 are decorated, marked with end-tool impressions. The 13 rim sherds derive from a Form 1A jar, and from vertical and everted bowls (Form 2B). A possible collar rim from a small vessel was found in Spit 3. Rim profiles are parallel and thinning, and lips forms are flat, rounded and tapering. A single carination was present in Spit 6. 
Table 52. Volivoli II ceramics: Sherd type by excavation square and depth.

\begin{tabular}{|c|c|c|c|c|c|c|}
\hline Depth $(\mathrm{cm})$ & Plain body & Decorated body & Rims & Necks & Number & Weight (g) \\
\hline $0-10$ & 283 & & 5 & & 288 & 2023.2 \\
\hline $10-20$ & 310 & 1 & 4 & & 315 & 1695.5 \\
\hline $20-30$ & 292 & 4 & 7 & 2 & 305 & 1371.1 \\
\hline $30-40$ & 38 & 1 & 1 & 1 & 41 & 249.5 \\
\hline $40-50$ & 158 & 25 & 11 & 8 & 202 & 923.7 \\
\hline $50-60$ & 81 & 7 & 5 & 2 & 95 & 376.4 \\
\hline $60-70$ & 42 & & 1 & 1 & 44 & 135.2 \\
\hline $70-80$ & 24 & & & & 24 & 75.6 \\
\hline $80-90$ & 5 & & & & 5 & 21 \\
\hline \multicolumn{7}{|l|}{$90-100$} \\
\hline \multicolumn{7}{|l|}{$100-110$} \\
\hline $110-120$ & 2 & & & & 2 & 3.5 \\
\hline $120-130$ & 2 & & & & 2 & 8.6 \\
\hline Posthole fill & 3 & & & & 3 & 9.9 \\
\hline Total & 1240 & 38 & 34 & 14 & 1326 & 6893.2 \\
\hline
\end{tabular}

Table 53. Volivoli III ceramics: Sherd type by excavation square and depth.

\begin{tabular}{|c|c|c|c|c|c|c|}
\hline Depth (cm) & Plain body & Decorated body & Rims & Carination & Number & Weight (g) \\
\hline Surface & 28 & & 1 & & 29 & 283.5 \\
\hline \multicolumn{7}{|l|}{ TP.1:Spit 1} \\
\hline TP.1:Spit 2 & 50 & & 2 & & 52 & 480.2 \\
\hline TP.1:Spit 3 & 33 & 2 & 5 & & 40 & 258.9 \\
\hline TP.1:Spit 4 & 141 & & & & 141 & 1153.3 \\
\hline TP.1:Spit 5 & 15 & & 1 & & 16 & 161.4 \\
\hline TP.1:Spit 6 & 41 & & 3 & 1 & 45 & 274.1 \\
\hline TP.1:Spit 7 & 14 & & & & 14 & 66.7 \\
\hline TP.1:Spit 8 & 30 & & 1 & & 31 & 641.9 \\
\hline TP.1:Spit 9 & 3 & & & & 3 & 14.2 \\
\hline Total & 355 & 2 & 13 & & 371 & 3334.2 \\
\hline
\end{tabular}

\section{Kulu Bay ceramics}

A total of 11,398 sherds was recovered from the Kulu Bay excavations. Of these 10,353 (90.8\%) are plain body sherds, with about half of the total assemblage coming from the C11 excavation (Table 54). Lapita-age sherds marked with dentate stamping were found in all deposits, along with post-Lapita pottery marked with parallel and cross-hatch paddle impression (Table 55). Late-prehistoric ceramics, such as dari and comb-incised vessels, are represented by a few sherds. Lapita-era rim forms are from Form 1B jars with abruptly thickened 'collar' rims, and Form 1A jars with everted rims, including highly everted 'rolled' rims. As at Sigatoka, collar-rim jars are decorated with side-tool impressions and shell impressing along the collar, and carinations carry 
linear incision and dentate stamping. Many sherds have traces of red slip and wiping on internal and external surfaces. As well as Form 1B jars with a sub-globular body, and everted jars with short necks and everted jars with thickened rims, additional vessels include a probable flat-based dish, everted direct bowls, inverted direct bowls and indirect inverted bowls (Forms 2A, 2B, 2C). High sherd fragmentation prohibited vessel reconstruction and examples of rim forms and decoration are shown in Figures 127-131.

Table 54. Kulu Bay ceramics: Sherd type by excavation square and depth.

\begin{tabular}{|c|c|c|c|c|c|c|c|}
\hline Excavation/depth (cm) & Plain body & Decorated body & Rims & Carinations & Handles/ lugs & Number & Weight (g) \\
\hline TP2:0-20 & 9 & 10 & 11 & & & 30 & 768.4 \\
\hline TP2:10-20 & 97 & 13 & 10 & & & 120 & 698.1 \\
\hline TP2:30-40 & 117 & 3 & 6 & & & 126 & 870.11 \\
\hline (9:0-10 & 128 & 10 & 9 & & & 147 & 530.2 \\
\hline C9:10-20 & 105 & 1 & 4 & & & 110 & 297.2 \\
\hline C9:20-30 & 220 & 7 & 18 & & & 245 & 1265.6 \\
\hline C9:30-40 & 395 & 12 & 27 & & & 434 & 1672.6 \\
\hline C9:40-50 & 703 & 15 & 38 & & & 756 & 2255.6 \\
\hline C9:50-60 & 418 & 11 & 24 & 2 & & 455 & 1653.9 \\
\hline C10: $0-30$ & 151 & 7 & 12 & & & 170 & 1082 \\
\hline C10:30-50 & 552 & 11 & 52 & & 1 & 616 & 3519.2 \\
\hline C10:50-70 & 89 & 5 & 5 & & & 99 & 987.3 \\
\hline C10:60-80 & 291 & 15 & 13 & & & 319 & 994.5 \\
\hline C10:70-80 & 314 & 10 & 15 & & & 339 & 1243.6 \\
\hline C10:80-90 & 443 & 12 & 11 & & & 466 & 1621.6 \\
\hline C10:90-100 & 645 & 30 & 43 & & 1 & 719 & 2309.4 \\
\hline C11:0-10 & 166 & 8 & 9 & 3 & 1 & 187 & 922.1 \\
\hline C11:10-20 & 234 & 10 & 10 & & & 254 & 1003.8 \\
\hline C11:20-30 & 453 & 17 & 41 & 3 & & 514 & 2823.4 \\
\hline C11:30-40 & 135 & 6 & 14 & 1 & & 156 & 873.5 \\
\hline C11:40-50 & 151 & 12 & 16 & & & 179 & 1278.5 \\
\hline C11:40-60 & 139 & 3 & 14 & & & 156 & 1006.6 \\
\hline C11:50-60 & 51 & 1 & 6 & & 1 & 59 & 428.6 \\
\hline C11: $60-70$ & 258 & 8 & 14 & 1 & 2 & 283 & 1463.8 \\
\hline C11:70-80 & 863 & 17 & 44 & 1 & 1 & 926 & 3668.4 \\
\hline C11:80-90 & 906 & 19 & 57 & & 3 & 985 & 2878.4 \\
\hline C11:90-100 & 622 & 16 & 45 & & 1 & 684 & 2613.8 \\
\hline C11:100-110 & 462 & 5 & 46 & 2 & & 515 & 1964.8 \\
\hline C11:110-120 & 383 & 27 & 25 & & & 435 & 1477.6 \\
\hline C11:120-130 & 359 & 10 & 14 & & & 383 & 1236.3 \\
\hline C11:130-140 & 350 & 5 & 19 & & & 374 & 1194.9 \\
\hline C11:140-150 & 98 & 2 & 2 & & & 102 & 297.9 \\
\hline C11:150-160 & 46 & 5 & 4 & & & 55 & 230.6 \\
\hline Total & 10353 & 343 & 678 & 13 & 11 & 11398 & 47132.3 \\
\hline
\end{tabular}


Table 55. Kulu Bay ceramics: Sherd decoration by excavation square and depth.

\begin{tabular}{|c|c|c|c|c|c|c|c|c|c|}
\hline Excavation/depth (cm) & Plain body & Dentate & Wiped & Side tool & Dents & $\mathrm{CPI}-/ /$ & CPI-X & Incision & End tool \\
\hline $\mathrm{T} 2: 0-20$ & 9 & 1 & 0 & 0 & 0 & 1 & 8 & 0 & 0 \\
\hline $\mathrm{T} 2: 10-20$ & 97 & 0 & 0 & 0 & 0 & 0 & 13 & 0 & 0 \\
\hline T2:30-40 & 117 & 0 & 1 & 0 & 0 & 1 & 1 & 0 & 0 \\
\hline T2 Total & 223 & 1 & 1 & 0 & 0 & 2 & 22 & 0 & 0 \\
\hline C9:0-10 & 128 & 2 & 3 & 0 & 0 & 0 & 5 & 0 & 0 \\
\hline C9:10-20 & 105 & 0 & 1 & 0 & 0 & 0 & 0 & 0 & 0 \\
\hline$C 9: 20-30$ & 220 & 1 & 1 & 0 & 0 & 2 & 3 & 0 & 0 \\
\hline C9:30-40 & 395 & 1 & 2 & 0 & 1 & 0 & 8 & 0 & 0 \\
\hline C9:40-50 & 703 & 4 & 4 & 1 & 0 & 1 & 5 & 0 & 0 \\
\hline C9:50-60 & 418 & 1 & 3 & 1 & 0 & 4 & 1 & 1 & 0 \\
\hline C9 Total & 1841 & 7 & 11 & 2 & 1 & 7 & 17 & 1 & 0 \\
\hline C10: $0-30$ & 151 & 0 & 1 & 0 & 1 & 2 & 3 & 0 & 1 \\
\hline C10:30-50 & 552 & 0 & 0 & 2 & 1 & 4 & 3 & 1 & 0 \\
\hline C10:50-70 & 89 & 1 & 1 & 1 & 0 & 0 & 2 & 0 & 0 \\
\hline C10:60-80 & 291 & 2 & 6 & 0 & 0 & 0 & 2 & 1 & 4 \\
\hline C10:70-80 & 314 & 2 & 3 & 2 & 1 & 1 & 1 & 0 & 0 \\
\hline C10:80-90 & 443 & 3 & 2 & 1 & 0 & 1 & 3 & 2 & 0 \\
\hline C10:90-100 & 645 & 2 & 6 & 1 & 0 & 3 & 14 & 2 & 2 \\
\hline C10 Total & 2485 & 10 & 19 & 7 & 3 & 11 & 28 & 6 & 7 \\
\hline C11:0-10 & 166 & 0 & 0 & 1 & 0 & 0 & 4 & 3 & 0 \\
\hline C11:10-20 & 234 & 0 & 2 & 2 & 0 & 2 & 3 & 1 & 0 \\
\hline C11:20-30 & 453 & 0 & 5 & 1 & 0 & 2 & 8 & 0 & 1 \\
\hline C11:30-40 & 135 & 0 & 4 & 0 & 0 & 0 & 2 & 0 & 0 \\
\hline C11:40-50 & 151 & 0 & 4 & 2 & 0 & 0 & 4 & 1 & 1 \\
\hline C11:40-60 & 139 & 0 & 0 & 0 & 0 & 0 & 3 & 0 & 0 \\
\hline C11:50-60 & 51 & 0 & 1 & 0 & 0 & 0 & 0 & 0 & 0 \\
\hline C11: $60-70$ & 258 & 2 & 2 & 0 & 0 & 1 & 3 & 0 & 0 \\
\hline C11:70-80 & 863 & 1 & 6 & 2 & 1 & 2 & 2 & 3 & 0 \\
\hline C11:80-90 & 906 & 1 & 13 & 1 & 0 & 0 & 3 & 0 & 0 \\
\hline C11:90-100 & 622 & 4 & 5 & 0 & 0 & 0 & 5 & 2 & 0 \\
\hline C11:100-110 & 462 & 0 & 2 & 0 & 0 & 0 & 3 & 0 & 0 \\
\hline C11:110-120 & 383 & 6 & 12 & 4 & 0 & 0 & 2 & 3 & 0 \\
\hline C11:120-130 & 359 & 0 & 3 & 3 & 0 & 1 & 3 & 0 & 0 \\
\hline C11:130-140 & 350 & 0 & 2 & 1 & 0 & 1 & 1 & 0 & 0 \\
\hline C11:140-150 & 98 & 0 & 0 & 1 & 0 & 0 & 0 & 1 & 0 \\
\hline C11:150-160 & 46 & 0 & 2 & 1 & 0 & 1 & 1 & 0 & 0 \\
\hline C11 Total & 5676 & 14 & 63 & 19 & 1 & 10 & 47 & 14 & 2 \\
\hline Total & 10353 & 34 & 97 & 28 & 5 & 30 & 119 & 21 & 9 \\
\hline
\end{tabular}




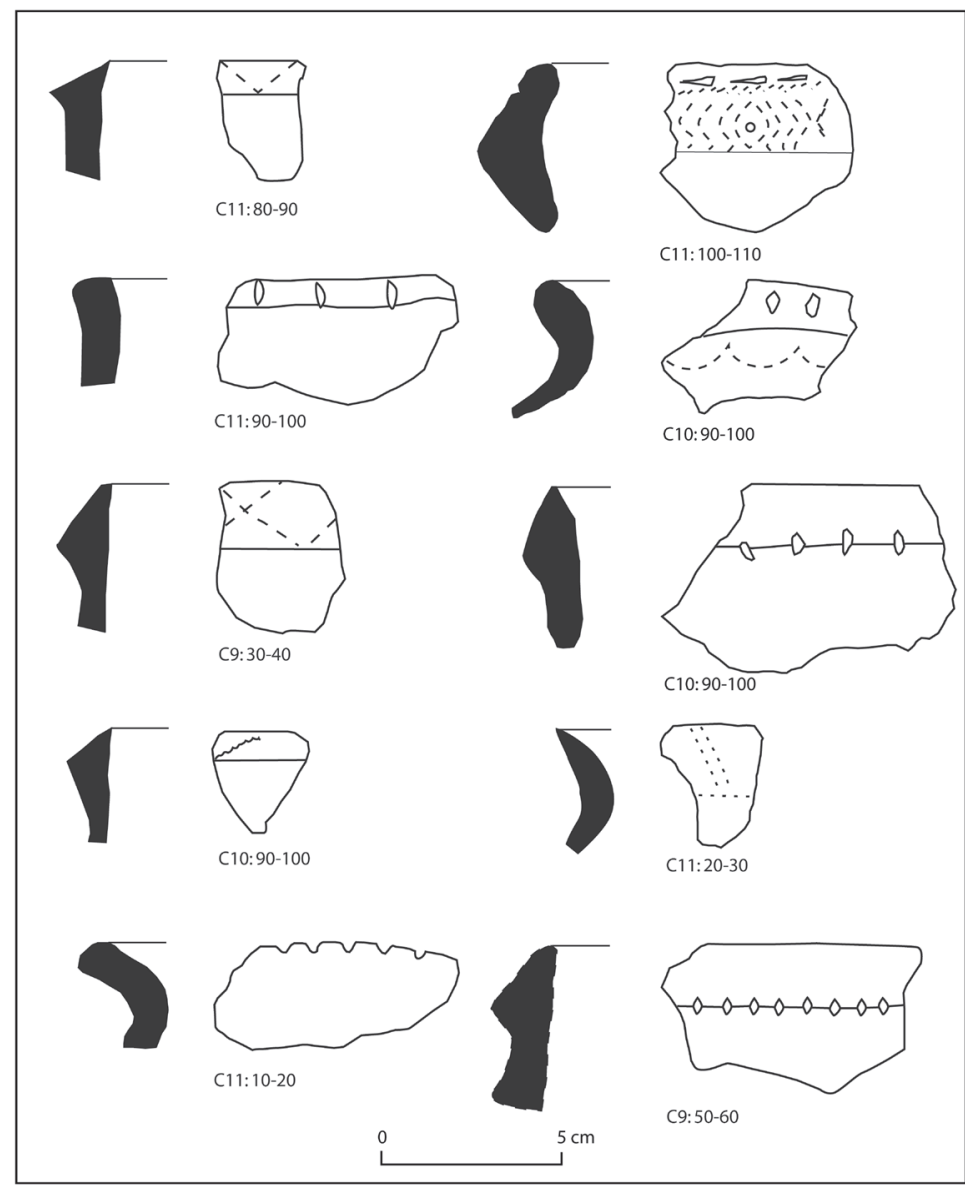

Figure 127. (Left) Kulu Bay decorated rim sherds.

Figure 128. (Below left) Kulu Bay rims and an incised carination.

Figure 129. (Below right) Kulu Bay decorated sherds.
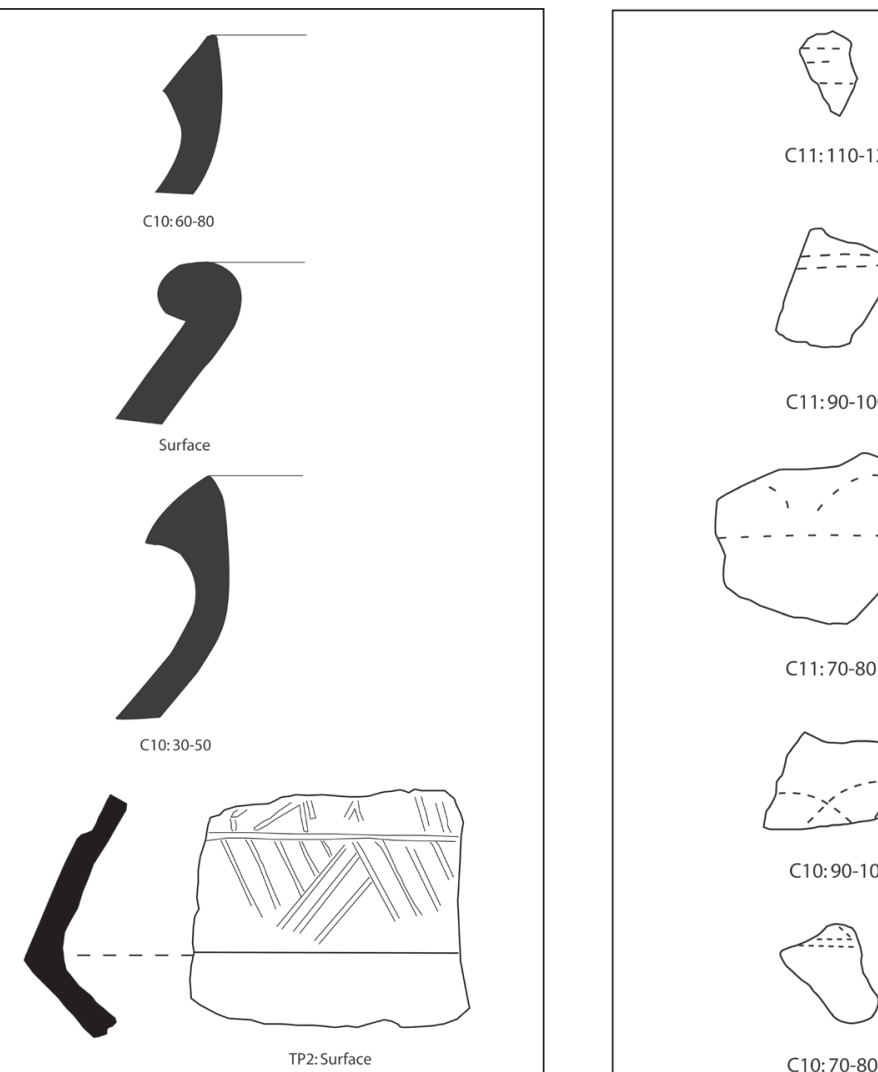

C11:110-120
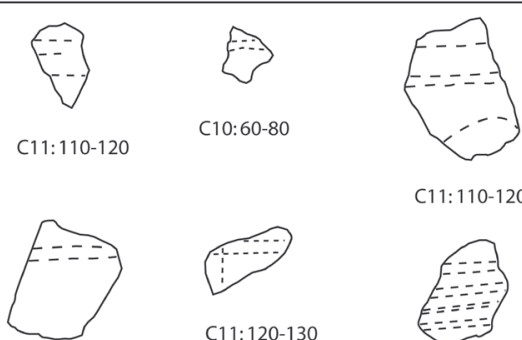

C11:110-120
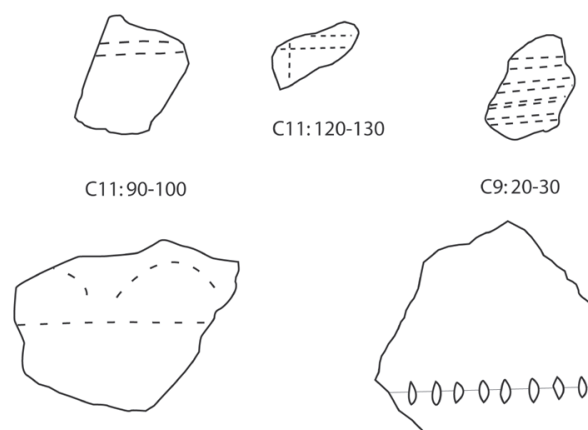

C11:70-80

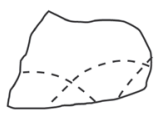

C9: 20-30

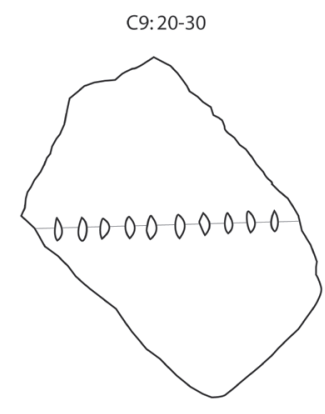

C10:30-50

C10:90-100
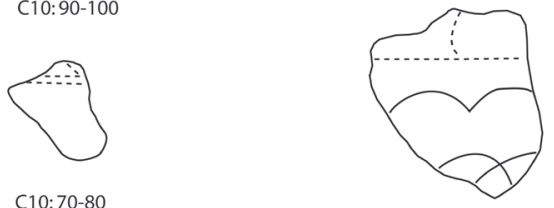

C10:70-80 $5 \mathrm{~cm}$ C10:80-90 


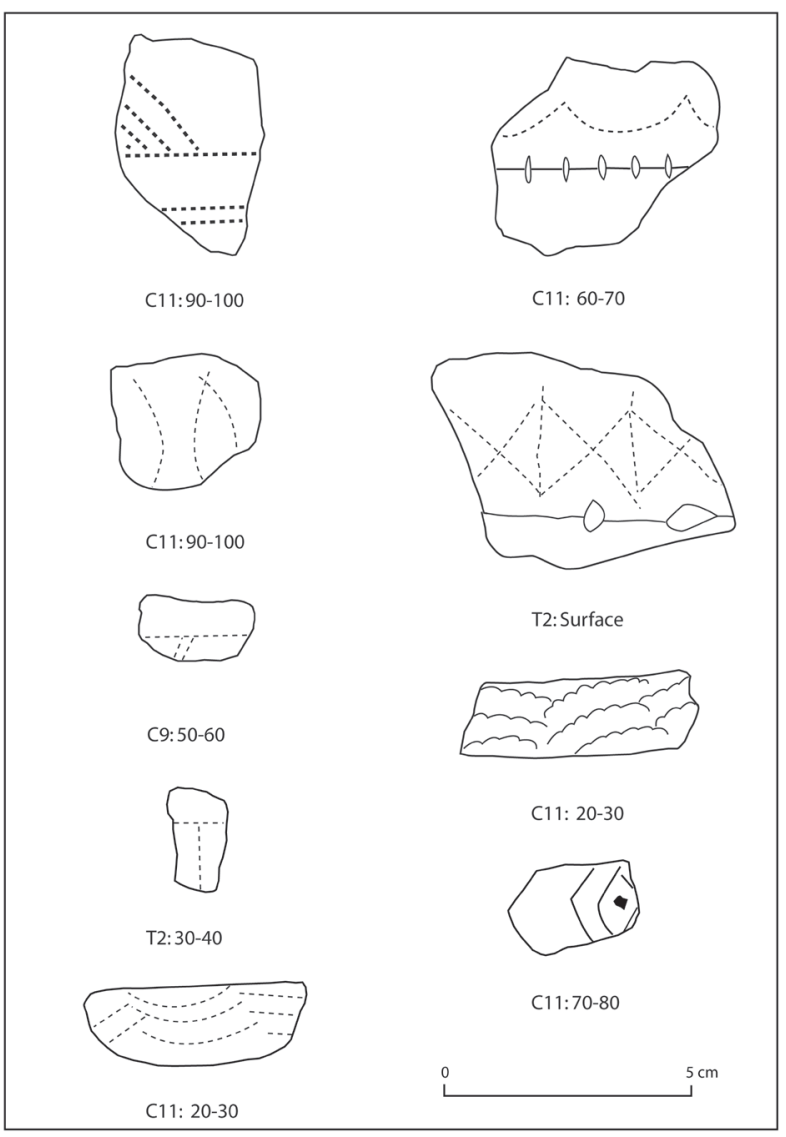

Figure 130. Kulu Bay decorated sherds.

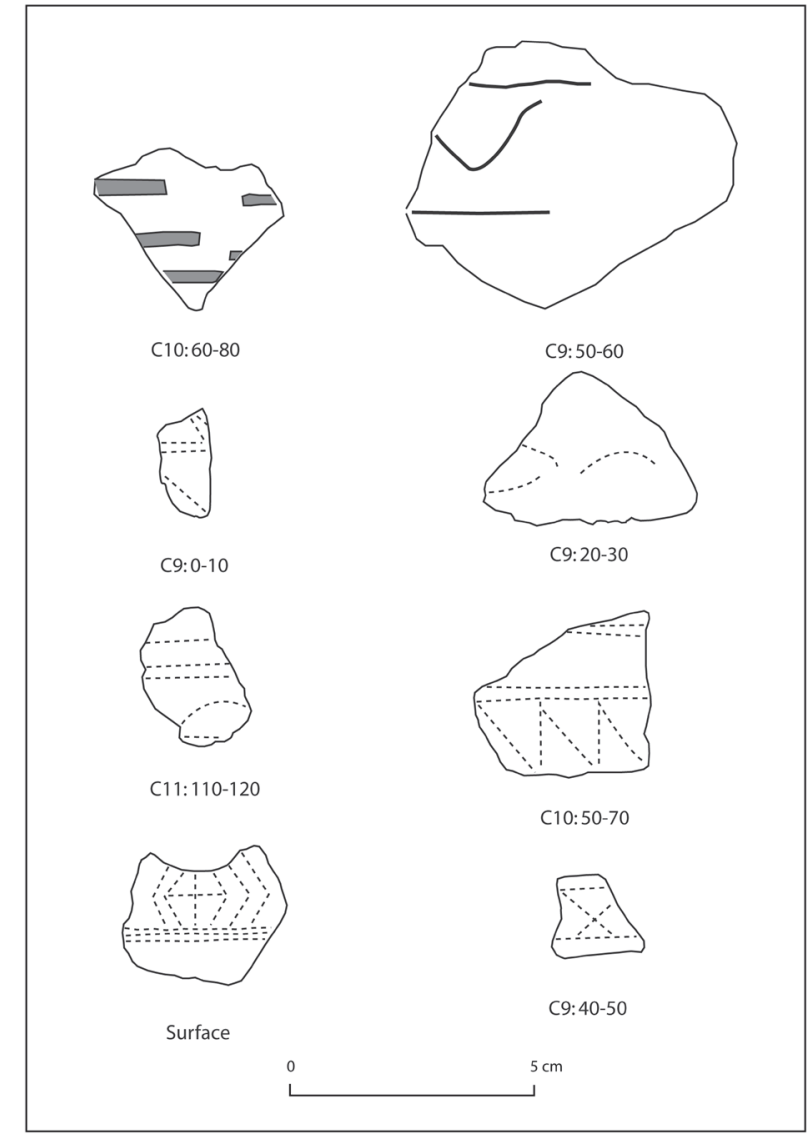

Figure 131. Kulu Bay decorated sherds.

\section{Natunuku ceramics}

The Natunuku investigations recovered 6318 sherds, weighing $17.13 \mathrm{~kg}$, with the majority of sherds deriving from the Trench 3 excavation. Sherd density is highest in the upper levels of TP1, TP2 and TR3, and all units contain decorated sherds from Lapita, post-Lapita and late-prehistoric assemblages (Table 56). This is demonstrated by comb-incised sherds in the upper deposits of TP1 and TP2, and by carved-paddle-impressed sherds in all levels of the TR3 excavations (Table 57). The highest frequency of dentate-stamped sherds was in the top $20 \mathrm{~cm}$ of units, except in TR3 where there were more decorated sherds than plain sherds at 40-50 cm depth. However, carved-paddle-impressed sherds were found in the lower levels of TR3, indicating deposit mixing of Lapita with post-Lapita ceramics has taken place. Dentatestamped decoration is similar to that reported by Davidson et al. (1990:Figures 12, 14, 15, 16) and examples are shown in Figure 132. Decorated sherds from the Natunuku assemblage were photographed and drawn, but after the 2003 Canberra bushfires, the Natunuku ceramics and a number of documentary records about the site could not be relocated, limiting the detail in which the collection can be described.

Nonetheless, it is possible from rim information to describe the main vessel forms and two complementary systems have been used. The first uses the lip shape and rim profile codes of Best (1984:161) for rims, while vessel-form categories follow those of previous work on Natunuku ceramics developed by Davidson et al. (1990). The latter study identified five vessel categories containing 15 vessel forms: Category i - flat-bottomed dish; Category ii - globular or ovoidbodied pot or jar; Category iii - water jar (narrow aperture); Category iv - shouldered pot/jar; 
Category v - direct bowl. Early vessels in the current study include Category i; Category ii Form IV (collar rim), Form VI and Form VII (outward flaring rim), Form VII (thickened rim); Category iv - Form XI, Form XIV (carinated), Form XIII (double flange rim). Late vessel forms are category ii - Form III, characterised by straight everted rims like those of late prehistoric kuro pots. Selected rim sherds are identified to a vessel category in Table 58, with category i (flat-bottomed dish) and category ii (Form VI and Form VII) collar-rim vessels and flaring-rim vessels from the Lapita assemblage, and category ii (Form III) vessels with straight everted rims of post-Lapita age.

Table 56. Natunuku ceramics: Sherd type by excavation square and depth.

\begin{tabular}{|c|c|c|c|c|c|c|}
\hline Excavation/depth (cm) & Plain body & Decorated body & Plain rims & Decorated rims & Handles/lugs & Weight (g) \\
\hline TP1:Surface & 11 & 0 & 2 & 0 & 1 & 140.9 \\
\hline TP1:0-10 & 416 & 27 & 14 & 7 & 0 & 1375.7 \\
\hline TP1:10-20 & 49 & 2 & 0 & 0 & 0 & 240.5 \\
\hline TP1/2:0-10 & 107 & 6 & 4 & 0 & 1 & 333.3 \\
\hline TP1/2:10-20 & 97 & 5 & 8 & 0 & 0 & 221.8 \\
\hline TP1/2:20-30 & 132 & 1 & 0 & 0 & 0 & 232.2 \\
\hline Total & 812 & 41 & 28 & 7 & 2 & 2544.4 \\
\hline TP2:0-10 & 590 & 35 & 16 & 5 & 1 & 1943.6 \\
\hline TP2:10-20 & 628 & 13 & 11 & 2 & 2 & 1531.2 \\
\hline TP2-1:20-30 & 69 & 1 & 0 & 3 & 0 & 213.2 \\
\hline TP2-1:30-40 & 5 & 0 & 0 & 0 & 0 & 5.3 \\
\hline Total & 1292 & 49 & 27 & 10 & 3 & 3693.3 \\
\hline TR3:0-10 & 1016 & 23 & 36 & 4 & 3 & 2482.9 \\
\hline TR3:10-20 & 879 & 14 & 32 & 7 & 0 & 2686.9 \\
\hline TR3:20-30 & 660 & 16 & 27 & 1 & 1 & 1965.5 \\
\hline TR3:30-40 & 604 & 9 & 35 & 1 & 1 & 1883.3 \\
\hline TR3:40-50 & 418 & 13 & 25 & 4 & 0 & 1274.9 \\
\hline TR3:50-60 & 141 & 2 & 7 & 0 & 0 & 422 \\
\hline TR3:Feature A & 46 & 3 & 2 & 0 & 0 & 134.7 \\
\hline No depth & 17 & 0 & 0 & 0 & 0 & 45 \\
\hline Total & 3781 & 80 & 164 & 17 & 5 & 10895.2 \\
\hline Site Total & 5885 & 170 & 219 & 34 & 10 & 17132.9 \\
\hline
\end{tabular}


Table 57. Natunuku ceramics: Sherd decoration by excavation square and depth.

\begin{tabular}{|c|c|c|c|c|c|c|c|c|c|}
\hline Excavation/depth (cm) & Plain & Dentate & Wiped & End tool & Appliqué & CPI-// & CPI-X & Incised & Comb Incised \\
\hline TP1:Surface & 11 & 0 & 0 & 0 & 0 & 0 & 0 & 0 & 1 \\
\hline TP1:0-10 & 416 & 12 & 2 & 2 & 1 & 3 & 2 & 4 & 8 \\
\hline TP1:10-20 & 49 & 0 & 0 & 0 & 0 & 0 & 1 & 0 & 1 \\
\hline TP1/2:0-10 & 107 & 3 & 0 & 0 & 1 & 0 & 1 & 0 & 1 \\
\hline TP1/2:10-20 & 97 & 2 & 0 & 1 & 0 & 0 & 1 & 0 & 1 \\
\hline TP1/2:20-30 & 132 & 0 & 0 & 0 & 0 & 0 & 0 & 0 & 1 \\
\hline TP2:0-10 & 590 & 31 & 2 & 3 & 0 & 1 & 0 & 2 & 1 \\
\hline TP2:10-20 & 628 & 10 & 0 & 1 & 1 & 1 & 1 & 0 & 1 \\
\hline TP2:20-30 & 69 & 2 & 0 & 2 & 0 & 0 & 0 & 0 & 0 \\
\hline TP2:30-40 & 5 & 0 & 0 & 0 & 0 & 0 & 0 & 0 & 0 \\
\hline TR3:0-10 & 1016 & 16 & 2 & 4 & 1 & 0 & 2 & 1 & 1 \\
\hline TR3:10-20 & 879 & 12 & 0 & 5 & 0 & 0 & 4 & 0 & 0 \\
\hline TR3:20-30 & 660 & 6 & 1 & 2 & 0 & 1 & 7 & 0 & 0 \\
\hline TR3:30-40 & 604 & 4 & 2 & 2 & 0 & 1 & 1 & 0 & 0 \\
\hline TR3:40-50 & 418 & 12 & 2 & 0 & 1 & 2 & 0 & 0 & 0 \\
\hline TR3:50-60 & 141 & 1 & 0 & 0 & 0 & 0 & 1 & 0 & 0 \\
\hline TR3:Feature A & 46 & 1 & 0 & 0 & 0 & 2 & 0 & 0 & 0 \\
\hline TR3:No Depth & 17 & 0 & 0 & 0 & 0 & 0 & 0 & 0 & 0 \\
\hline Site Total & 5885 & 112 & 11 & 22 & 5 & 11 & 21 & 7 & 16 \\
\hline
\end{tabular}

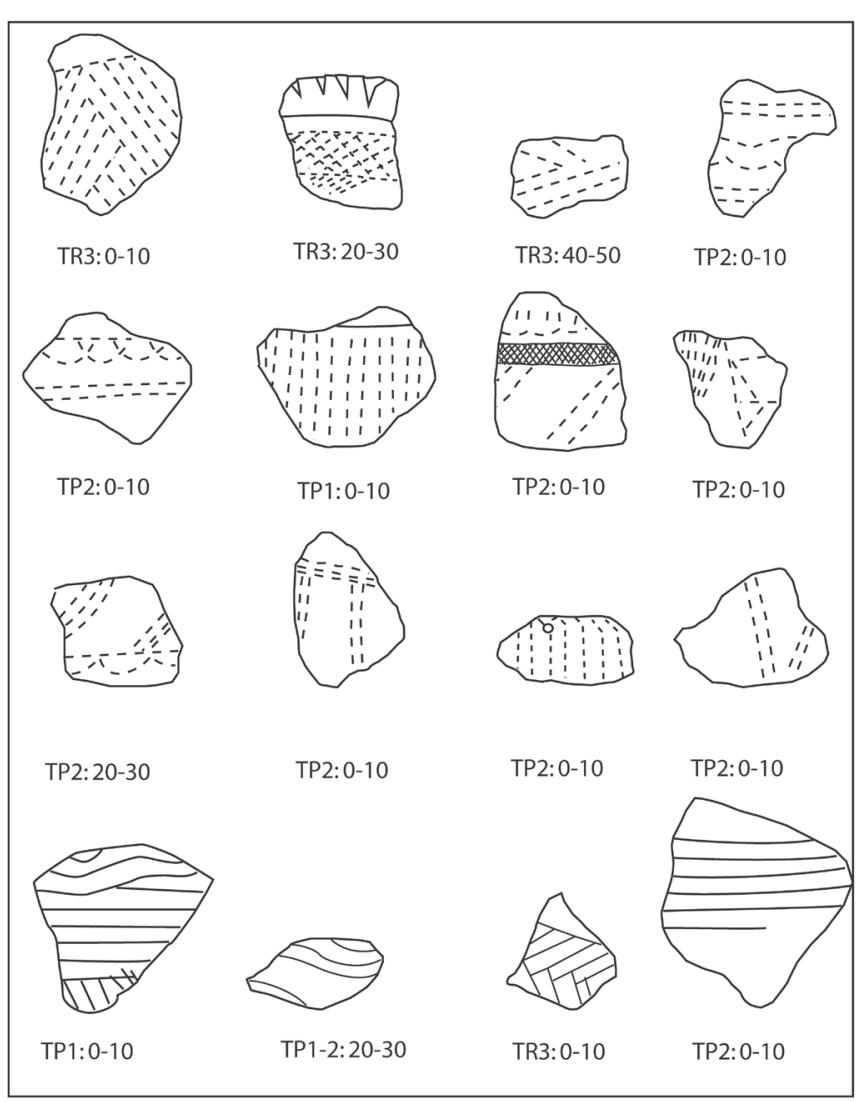

Figure 132. Natunuku decorated sherds. 
Table 58. Natunuku ceramics: Selected vessel forms and rims by excavation square and depth.

\begin{tabular}{|c|c|c|c|c|c|}
\hline Excavation/depth (cm) & Lip & Profile & Decoration & Vessel type & Weight (g) \\
\hline TP1:0-10 & 39 & 30 & CPI-X & $?$ & 4.2 \\
\hline TP1:0-10 & 40 & 42 & Side tool & $?$ & 4.6 \\
\hline TP1:0-10 & 47 & 35 & Dentate & Category iv & 9.7 \\
\hline TP1:0-10 & 40 & 30 & Dentate & Category i & 6.1 \\
\hline TP1:0-10 & 39 & 30 & Dentate & Category i & 10.2 \\
\hline TP1:0-10 & 49 & 34 & Side tool & Category iv & 6.2 \\
\hline TP2:0-10 & 45 & 30 & Side tool & $?$ & 7.2 \\
\hline TP2:0-10 & 39 & 30 & Dentate & ?Category i & 2.8 \\
\hline TP2:0-10 & 40 & 30 & Dentate & ?Category i & 3.3 \\
\hline TP2:0-10 & 39 & 30 & Dentate & ?Category i & 4.8 \\
\hline TP2:10-20 & 40 & 33 & End tool & $?$ & 4.7 \\
\hline TP2:10-20 & 39 & 30 & Dentate & Category i & 31.0 \\
\hline TP2/1:20-30 & 40 & 30 & Dentate+Side tool & ?Category i & 5.5 \\
\hline TP2/1:20-30 & 40 & 30 & Side tool & Category ii & 6.7 \\
\hline TP2/2:0-10 & 42 & 34 & Side tool & Category iv & 72.9 \\
\hline TP2/2:20-30 & 39 & 30 & Dentate+Side tool & ?Category i & 3.3 \\
\hline $\mathrm{TR} 3 / \mathrm{A} 3 / 2 \mathrm{~A}$ & 39 & $?$ & Side tool & $?$ & 1.7 \\
\hline TR3/A4:0-10 & 40 & 30 & Side tool & $?$ & 3.9 \\
\hline TR3/A4:10-20 & 39 & 30 & Dentate & ?Category i & 7.2 \\
\hline TR3/A4:20-30 & 40 & 30 & Perforation & $?$ & 2.0 \\
\hline TR3/A5:0-10 & 39 & 31 & Side tool & $?$ & 2.4 \\
\hline TR3/A5:0-10 & 44 & 30 & Dentate & Category i & 2.9 \\
\hline TR3/A6:10-20 & 44 & 30 & Side tool & $?$ & 3.5 \\
\hline TR3/A6:10-20 & 39 & 31 & Side tool & $?$ & 6.1 \\
\hline TR3/A6:10-20 & 39 & 30 & Side tool & $?$ & 5.4 \\
\hline TR3/A6:20-30 & 39 & 30 & Side tool & $?$ & 8.1 \\
\hline TR3/A6:30-40 & 39 & 30 & Dentate+Side tool & Category i & 2.5 \\
\hline TR3/A6:40-50 & 39 & 30 & Side tool & $?$ & 9.9 \\
\hline TR3/A6:40-50 & 39 & 30 & (PI-// & $?$ & 1.1 \\
\hline TR3/A6:40-50 & 44 & 30 & Dentate & ?Category i & 2.6 \\
\hline TR3/A6:40-50 & 44 & 30 & Side tool & $?$ & 2.7 \\
\hline TR3/B6:10-20 & 39 & 30 & Side tool & $?$ & 2.4 \\
\hline TR3/B6:10-20 & 39 & 30 & Side tool & $?$ & 3.3 \\
\hline TP1:0-10 & 39 & 30 & Plain & Category v & 2.4 \\
\hline TP1:0-10 & 49 & 30 & Plain & Category v & 2.7 \\
\hline TP1:0-10 & 40 & 30 & Plain & Category v & 5.5 \\
\hline TP1:0-10 & 49 & 30 & Plain & Category v & 7.1 \\
\hline TP1:0-10 & 39 & 30 & Plain & Category v & 11.0 \\
\hline
\end{tabular}


Table 58 continued

\begin{tabular}{|c|c|c|c|c|c|}
\hline Excavation/depth $(\mathrm{cm})$ & Lip & Profile & Decoration & Vessel type & Weight (g) \\
\hline TP1:0-10 & 39 & 30 & Plain & Category v & 23.7 \\
\hline TP2:10-20 & 39 & 30 & Plain & Category v & 7.9 \\
\hline TR3/A5:0-10 & 40 & 30 & Plain & Category v & 2.6 \\
\hline TR3/A5:10-20 & 39 & 30 & Plain & Category v & 15.4 \\
\hline TR3/B5:10-20 & 39 & 30 & Plain & Category v & 11.2 \\
\hline TR3/B5:30-40 & 45 & 30 & Plain & Category v & 14.5 \\
\hline TR3/B5:40-50 & 39 & 30 & Plain & Category v & 3.9 \\
\hline TR3/B5:40-50 & 40 & 30 & Plain & Category v & 4.7 \\
\hline TR3/B6:50-60 & 40 & 30 & Plain & Category v & 6.4 \\
\hline TP1:Surface & 39 & 30 & Plain & Category ii-III & 9.6 \\
\hline TP1:0-10 & 44 & 31 & Plain & Category ii-III & 4.9 \\
\hline TP2:10-20 & 39 & 32 & Plain & Category ii-VII & 14.7 \\
\hline TR3/A4:0-10 & 45 & 30 & Plain & Category ii-III & 2.5 \\
\hline TR3/A5:30-40 & 39 & 35 & Plain & Category ii-III & 14.4 \\
\hline TR3/A5:30-40 & 39 & 33 & Plain & Category ii-VII & 10.3 \\
\hline TR3/A5:30-40 & 39 & 30 & Plain & Category ii-III & 9.4 \\
\hline TR3/A6:20-30 & 45 & 30 & Plain & Category ii-VI & 5.7 \\
\hline TR3/A6:40-50 & 40 & 31 & Plain & Category ii-III & 3.5 \\
\hline TR3/A6:40-50 & 39 & 30 & Plain & Category ii-III & 6.5 \\
\hline TR3/B6:40-50 & 39 & 30 & Plain & Category i & 19.9 \\
\hline
\end{tabular}

'Lip' and 'Profile' descriptions after Best (1984:170, Fig. 3.3). Vessel categories and forms after Davidson et al. (1990).

\section{Qaranioso II ceramics}

The small Qaranioso II pottery assemblage consists of 206 sherds, weighing $0.93 \mathrm{~kg}$ (Table 59). Most are plain-body sherds, with carved-paddle-impressed sherds present in the middle levels, along with one sherd marked with fingernail impressions. At the base of the site was a rim sherd from a small vessel (estimated aperture diameter 16-18 cm), marked on the lip from a section of bivalve shell arc containing seven teeth (Figure 133). The rim is thickened at the lip and is similar to rims found at Ugaga and at Sigatoka Level 1 (cf. Birks 1973:Vessels No. 37 and 38).

Table 59. Qaranioso II ceramics: Sherd type and decoration by excavation square and depth.

\begin{tabular}{|c|c|c|c|c|c|c|c|}
\hline Depth & Plain body & Decorated body & Rims & Weight (g) & CPI-X & End tool - fingernail & Shell impressed \\
\hline TP1:0-10 & 6 & & & 105.0 & & & \\
\hline TP1:10-20 & 30 & 2 & 1 & 130.7 & 1 & 1 & \\
\hline TP1:20-30 & 53 & 7 & & 231.2 & 7 & & \\
\hline TP1:30-40 & 40 & & 4 & 234.7 & & & \\
\hline TP1:40-50 & 41 & & 2 & 152.8 & & & \\
\hline TP1:50-60 & 18 & 1 & 1 & 73.9 & & & 1 \\
\hline Total & 188 & 10 & 8 & 928.3 & 8 & 1 & 1 \\
\hline
\end{tabular}




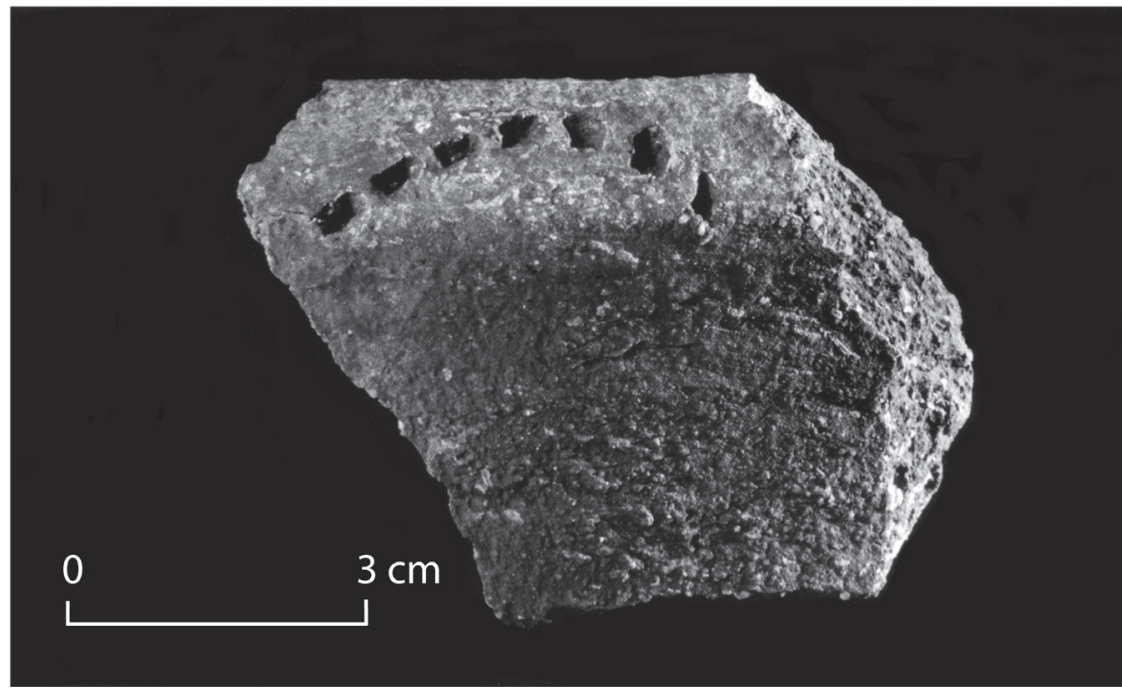

Figure 133. Qaranioso II rim sherd marked with a coarse dentate tool.

\section{References}

Anderson, A. and Clark, G. 1999. The age of Lapita settlement in Fiji. Archaeology in Oceania 34: 31-39. Anson, D. 1983. Lapita pottery of the Bismarck Archipelago and its affinities. Unpublished PhD thesis, University of Sydney.

Best, S. 1984. Lakeba: The prehistory of a Fijian Island. Unpublished PhD thesis, Department of Anthropology, University of Auckland.

Birks, L. 1973. Archaeological excavations at Sigatoka dune site, Fiji. Bulletin of the Fiji Museum No.1.

Clark, G. 2000. Post-Lapita Fiji: Cultural transformation in the mid-sequence. Unpublished PhD thesis, Australian National University.

Clark, G. and Anderson, A. 2001. The pattern of Lapita settlement in Fiji. Archaeology in Oceania 36: 77-88.

Clark, G., Anderson, A. and Matararaba, S. 2001. The Lapita site at Votua, northern Lau Islands, Fiji. Archaeology in Oceania 36: 134-145.

Crosby, A. 1988. Beqa: Archaeology, structure and history in Fiji. Unpublished MA thesis, Department of Anthropology, University of Auckland.

Davidson, J., Hinds, E., Holdaway, S. and Leach, F. 1990. The Lapita site of Natunuku, Fiji. New Zealand Journal of Archaeology 12: 121-155.

Frost, E.L. 1970. Archaeological excavations of fortified sites on Taveuni, Fiji. Unpublished PhD thesis, University of Oregon.

Gifford, E.W. 1951. Archaeological excavations in Fiji. University of California Anthropological Records 13:189-288.

Harrisson, T. 1965. 'Turtle-ware' from Borneo caves. The Sarawak Museum Journal 12: 63-67.

Lambert, R. 1971. Botanical identification of impressions on archaeological potsherds from Sigatoka. Final Report No. 3. Records of the Fiji Museum 1: 124-148.

Mead, S., Birks, L., Birks, H. and Shaw, E. 1973. The Lapita pottery style of Fiji and its associations. Journal of the Polynesian Society Memoir 38: 1-98.

Palmer, B. 1971. Fijian pottery technologies: their relevance to certain problems of southwest Pacific history. In: Green, R.C. and Kelly, M. (eds), Studies in Oceanic culture history volume 2, pp. 77-103. Pacific Anthropological Records No. 12.

Parham, J. 1964. Plants of the Fiji Islands. Government Press, Suva.

Rossitto, R. 1990. Stylistic change of Fijian pottery. Part 1: form. Domodomo 8: 1-47.

Shaw, E. 1967. A reanalysis of pottery from Navatu and Vuda, Fiji. Unpublished MA thesis, Department of Anthropology, University of Auckland.

Summerhayes, G.R. 1996. Interaction in Pacific prehistory: An approach based on production, distribution and use of pottery. Unpublished $\mathrm{PhD}$ thesis, La Trobe University. 